BRIDGE-SCOUR DATA FOR THE HIGHWAY 101 BRIDGE OVER ALSEA RIVER ESTUARY AT WALDPORT, OREGON -- 1988-90

By Milo D. Crumrine

U. S. GEOLOGICAL SURVEY

Open-File Report 91-531

Prepared in cooperation with

OREGON DEPARTMENT OF TRANSPORTATION 


\section{U. S. DEPARTMENT OF THE INTERIOR}

MANUEL LUJAN, JR., Secretary

\section{U.S. GEOLOGICAL SURVEY}

Dallas L. Peck, Director

For additional information write to:

\section{District Chief}

U.S. Geological Survey, WRD 10615 S.E. Cherry Blossom Drive

Portland, Oregon 97216
Copies of this report can be purchased from:

U.S. Geological Survey Books and Open-File Reports Section Federal Center, Box 25425

Denver, Colorado 80225 


\section{CONTENT}

Abstract

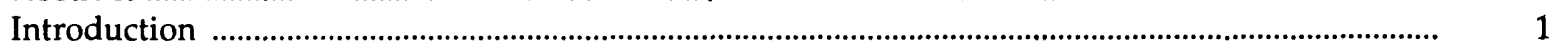

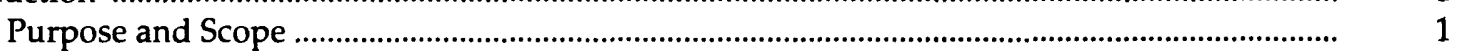

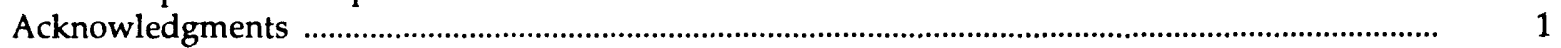

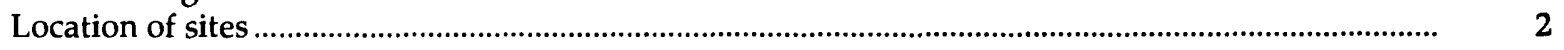

Data Collection ..................................................................................................................................... 2

Stage, velocity, and discharge ................................................................................................................

GeneraI, constriction, and local scour.................................................................................................. 2

Suspended-sediment and bed materiaI ................................................................................................ 4

Methods of data collection .........................................................................................................................

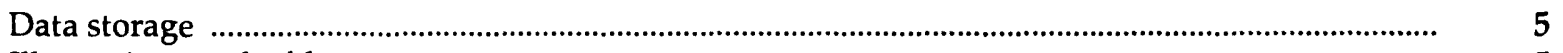

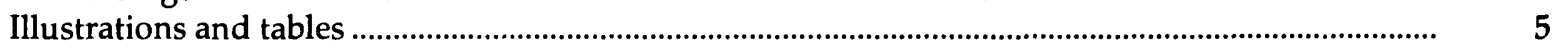

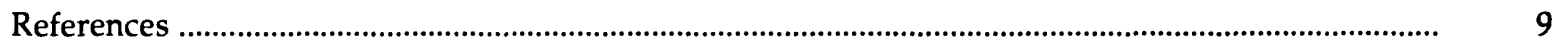

\section{ILLUSTRATIONS}

Figure 1. Area map showing study locations in the Alsea River Estuary

near Waldport, Oregon

2. Plan view of of Highway 101 bridge, monitoring Iocations, and construction

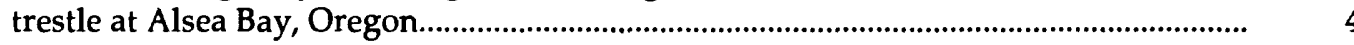

3. Bed elevations at bent 4, and reference location .......................................................................

4. Gage height and scour-hole elevation data at bent 4, July 1-4 and September 1-4, 1989..... 13

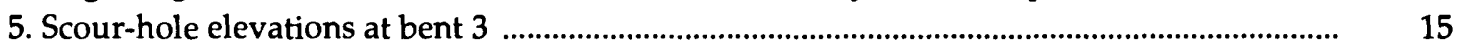

6. Gage height and scour-hole-elevation data at bent 3 for

7. Cross sections at Alsea Bay, 600 feet west of the old

Highway 101 Bridge piers ............................................................................................. $\quad 17$

8. Cross sections at Alsea Bay, 300 feet east of the old

Highway 101 Bridge piers ...................................................................................................... $\quad 18$

9. Cross sections at Alsea Bay, 20 feet west of the old

Highway 101 Bridge piers .............................................................................................. 19

10. Mean daily discharge for Alsea River near Tidewater for water years 1988-90 .................. 21

11. Water levels during high flow of the Alsea River at

Waldport, Drift Creek Landing, and Kozy Kove, January 6-8, 1990 ............................... $\quad 22$

12. Water levels during low flow of the Alsea River at

Waldport, Drift Creek Landing, and Kozy Kove, August 1-3, 1989 ............................... 22 
1. Begin and end dates and accuracy of continuous 15-minute data that will be stored in the Bridge Scour Data Base System .

2. Dates for cross-sectional measurements of the Alsea River at Waldport, Oregon

3. Scour-hole bed elevations for northeast corner of bent 4, Alsea River at Waldport

cour-hole bed elevations for southeast corner of bent 4, Alsea River

5. Daily scour-hole bed elevations for southwest corner of bent 4, Alsea River at Waldport.

6. Bed elevations for 40 feet east of bent 4 , Alsea River at Waldport..

7. Scour-hole bed elevations for southeast corner of bent 3, Alsea River at Waldport.

8. Maximum and minimum daily water levels for Alsea River at Waldport

9. Maximum and minimum daily water levels for Alsea River at Waldport

10. Maximum and minimum daily water levels for Alsea River at Kozy Kove near Waldport, river mile 11.2

11. Discharge measurements taken in the Alsea River Estuary.

CONVERSION FACTIORS AND VERTICAL DATUM

\begin{tabular}{|c|c|c|}
\hline Multiply & By & To obtain \\
\hline \multicolumn{3}{|c|}{ LENGTH } \\
\hline inch (in) & 25.4 & millimeter \\
\hline foot (ft) & 0.3048 & meter \\
\hline \multirow[t]{2}{*}{ mile (m) } & 1.609 & kilometer) \\
\hline & AREA & \\
\hline acre & 4,047 & square meter \\
\hline acre & 0.407 & hectare (ha) \\
\hline square mile $\left(\mathrm{mi}^{2}\right)$ & 259.0 & hectare (ha) \\
\hline square mile $\left(\mathrm{mi}^{2}\right)$ & 2.590 & square kilometer $\left(\mathrm{km}^{2}\right)$ \\
\hline & VOLUME & \\
\hline \multirow[t]{2}{*}{ cubic foot $\left(\mathrm{ft}^{3}\right)$} & 0.02832 & cubic meter $\left(\mathrm{m}^{3}\right)$ \\
\hline & FLOW & \\
\hline cubic foot per second $\left(\mathrm{ft}^{3} / \mathrm{s}\right)$ & 0.02832 & cubic meter per second $\left(\mathrm{m}^{3} / \mathrm{s}\right)$ \\
\hline gallon per minute (gal $/ \mathrm{min}$ ) & 0.06308 & liter per second $(\mathrm{L} / \mathrm{s})$ \\
\hline gallon per minute (gal $/ \mathrm{min}$ ) & 0.002228 & cubic foot per second $\left(\mathrm{ft}^{3} / \mathrm{s}\right)$ \\
\hline
\end{tabular}

SEA LEVEL: In this report "sea level" refers to the National Geodetic Vertical Datum of 1929 (NGVD of 1929)-- a geodetic datum derived from a general adjustment of the first-order level nets of both the United States and Canada, formerly called Sea Level Datum of 1929. 


\title{
BRIDGE SCOUR DATA FOR THE HIGHWAY 101 BRIDGE OVER ALSEA RIVER ESTUARY AT WALDPORT, OREGON -- 1988-90
}

\author{
By Milo D. Crumrine
}

\begin{abstract}
This report is a compilation of streambed scour and hydraulic data collected prior to and during the construction of a new bridge over the Alsea River Estuary at Waldport, Oregon. These data were collected in cooperation with the Oregon Department of Transportation for the purpose of determining maximum scour around cofferdams and bents of the Highway 101 Bridge (under construction) over Alsea Bay. Data collected includes (1) continuous water-level record of Alsea River at river mile 1.1, 5.0, and 11.2, (2) periodic continuous record of ebb and flood-tide velocities in the vicinity of bent 4 of the new bridge, (3) continuous record of bed elevations of Alsea Bay and selected scour holes at bent 3 and bent 4, (4) periodic cross-section measurements at several locations in the vicinity of the Highway 101 Bridge, (5) suspended sediment and bed-load sample results, and (6) cross sections at approximately 1 mile intervals from the mouth of Alsea River to river mile 12.5.
\end{abstract}

\section{INTRODUCTION}

Scour around bridge piers and abutments is a serious problem in many rivers and estuaries. Bridge failure is commonly caused by scour which undermines piers or abutments. Few data are available that document scour around bridge piers in estuaries. A bridge under construction from 1989 to 1991 at Alsea Bay near Waldport, Oregon, provided an excellent opportunity to collect data on bridge scour before, during and after construction.

The U.S. Geological Survey (USGS), in cooperation with Oregon Department of Transportation (ODOT), Bridge Section, started a project in 1988 to study scour through the construction period, in the vicinity of the new Highway 101 Bridge over Alsea Bay.

\section{Purpose and Scope}

The purpose of this report is to present data that documents the magnitude of scour around cofferdams and bents of a bridge under construction and to present associated data describing watersurface elevation, velocity, suspended sediment, and bed load. This report presents the types of data collected, the methods of collection, what data are available, and where the data can be obtained.

\section{Acknowledgments}

The author would like to acknowledge the Bridge Section of Oregon Department of Transportation, in particular, Tom Lulay and Dave Bryson for their support and interest in this project. The author would also like to thank George Hopkins, Phil Rabb and their crew at the Oregon Department of Transportation Bridge Office at Waldport for their help in surveying, monitoring equipment, and serving as liaison with the contractors and the USGS. Thanks also to General Construction, the contractors for the Waldport Bridge, for their willingness to help in this data collection effort. 


\section{LOCATION OF SITES}

The study area is located near the mouth of the Alsea River, which flows into the Pacific Ocean at Waldport, Oregon. The bridge being studied is currently (1991) under construction and is located about 100 $\mathrm{ft}$ (feet) upstream from the old Highway 101 Bridge at Waldport, Oregon, at river mile (RM) 1.10 (fig. 1).

The initial data collection site was located at pier 6 of the old bridge. Data collected at this site are water surface elevation, flood,and ebb tide velocities (fig. 2). After construction of bent 4 of the new bridge began, another data-collection site was established on the work trestle near the northeast corner of bent 4 , and was used to collect ebb-tide velocities, reference bed elevations, and scour-hole elevations at the northeast, southeast, and southwest corners of bent 4 . Two water surface elevation sites were established upstream at RM 5.0 and 11.2 (fig. 1). Another site was established at bent 3 to collect scour-hole-elevation data. Data collected at bent 3 was compared with data from bent 4 to evaluate spatial variability of scour between these two locations.

\section{DATA COLLECTION}

\section{Stage, Velocity, and Discharge}

Water surface elevation in Alsea Bay was measured at three locations to define the tidal prism of the estuary: at Highway 101 Bridge (RM 1.1), at confluence of Drift Creek (RM 5.0), and at Kozy Kove (RM 11.2). Ebb and flood-tide velocity was monitored at the Highway 101 Bridge in the main portion of flow near bent 4 . Periodic water discharge measurements were made to determine tidal flux. Measurements were made near the mouth of Alsea Bay during ebb and flood tides. One discharge measurement was made near the new bridge location during maximum high-tide conditions, and one measurement was made at RM 5.0, upstream from the main body of the estuary. Additional discharge data were available at the Alsea River near Tidewater streamflow measuring station at RM 21.0, about 8 miles upstream from the tidally affected area.

\section{General, Contraction, and Local Scour}

Scour that occurs around bridge piers is a combination of three types of scour (Jarrett and Boyle, 1986): (1) general scour or fill, that is degradation or aggradation of the streambed under normal conditions; (2) contraction scour caused by the presence of the bridge abutments and piers constricting streamflow, thus causing an increased velocity and degradation of the streambed in the vicinity of the bridge; and (3) local scour, caused by local disturbances of the flow, such as vortices and eddies around piers and abutments. Scour around bridge piers and footings in an estuary with strong tidal currents both upstream and downstream, such as the study area, further complicates the scour process and makes determination of scour around piers and abutments more difficult.

Scour data were collected to define general, contraction, and local scour in the study area. General scour was monitored by surveying cross sections parallel to the old Highway 101 Bridge at eight locations, beginning $600 \mathrm{ft}$ east of the bridge centerline and ending $600 \mathrm{ft}$ west. Constaction scour was monitored by recording bed elevations of Alsea Bay at the construction site, but out of the local scour area. Contraction scour near bent 4 was determined by continuously monitoring bed elevations about $40 \mathrm{ft}$ upstream from the cofferdam. This location was affected by contraction scour, but was not affected by local scour from bent 4. Local scour was monitored at each corner of bent 4 of the cofferdam. A second site (bent 3 ) was monitored for comparison with local scour at bent 4 .

Local scour at bent 4 was monitored on a continuous basis at the southwest corner and northeast or southeast corner of the cofferdam. Periodic measurements were made at each corner of the cofferdam at bent 4 . Normally, local scour depths would be monitored at the upstream corners of bridge piers; however, at this site, water flows in both directions due to tides, and downstream corners also needed to be monitored because of scour caused by flood tides. Local scour measurements made at bent 4 were 


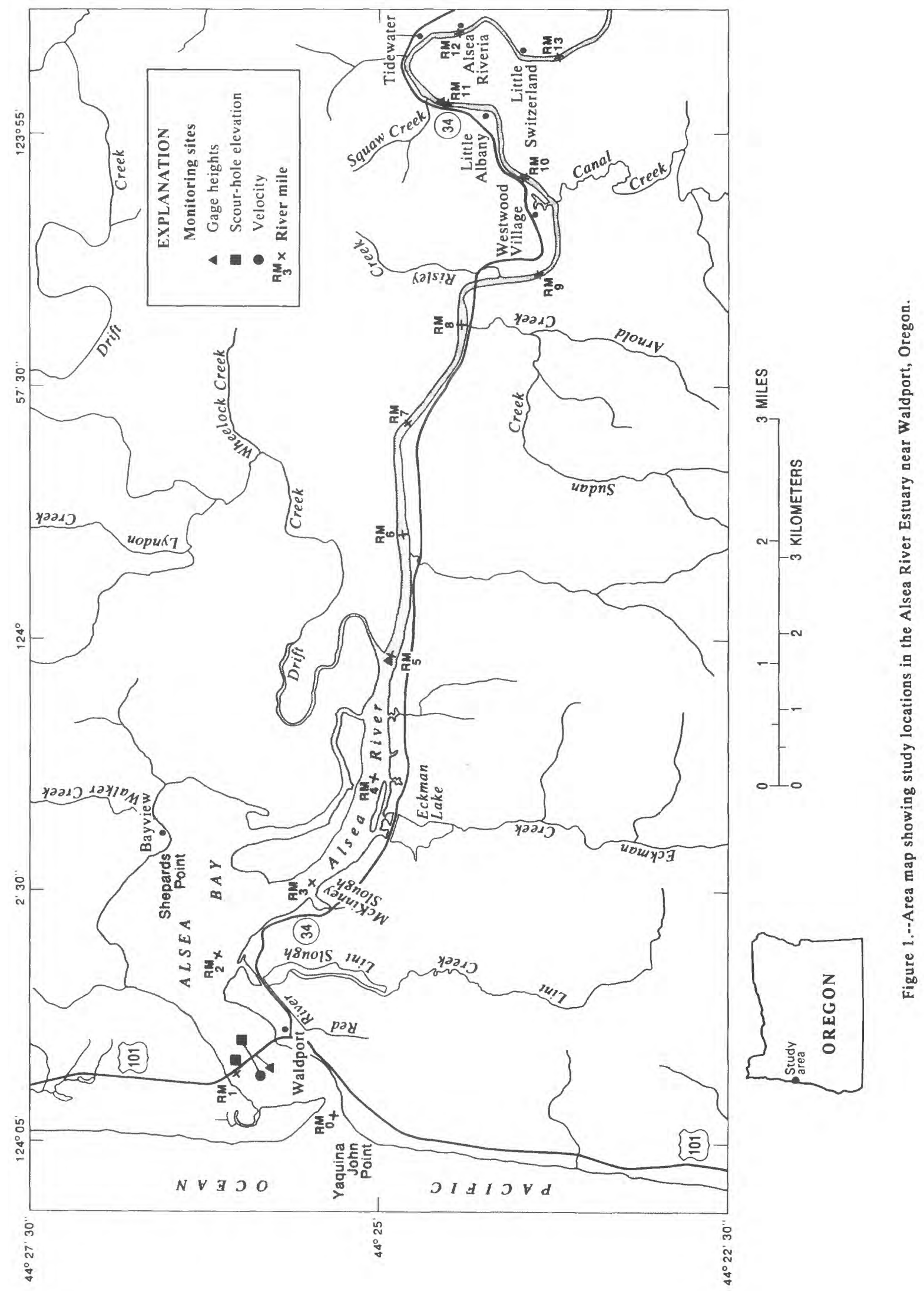




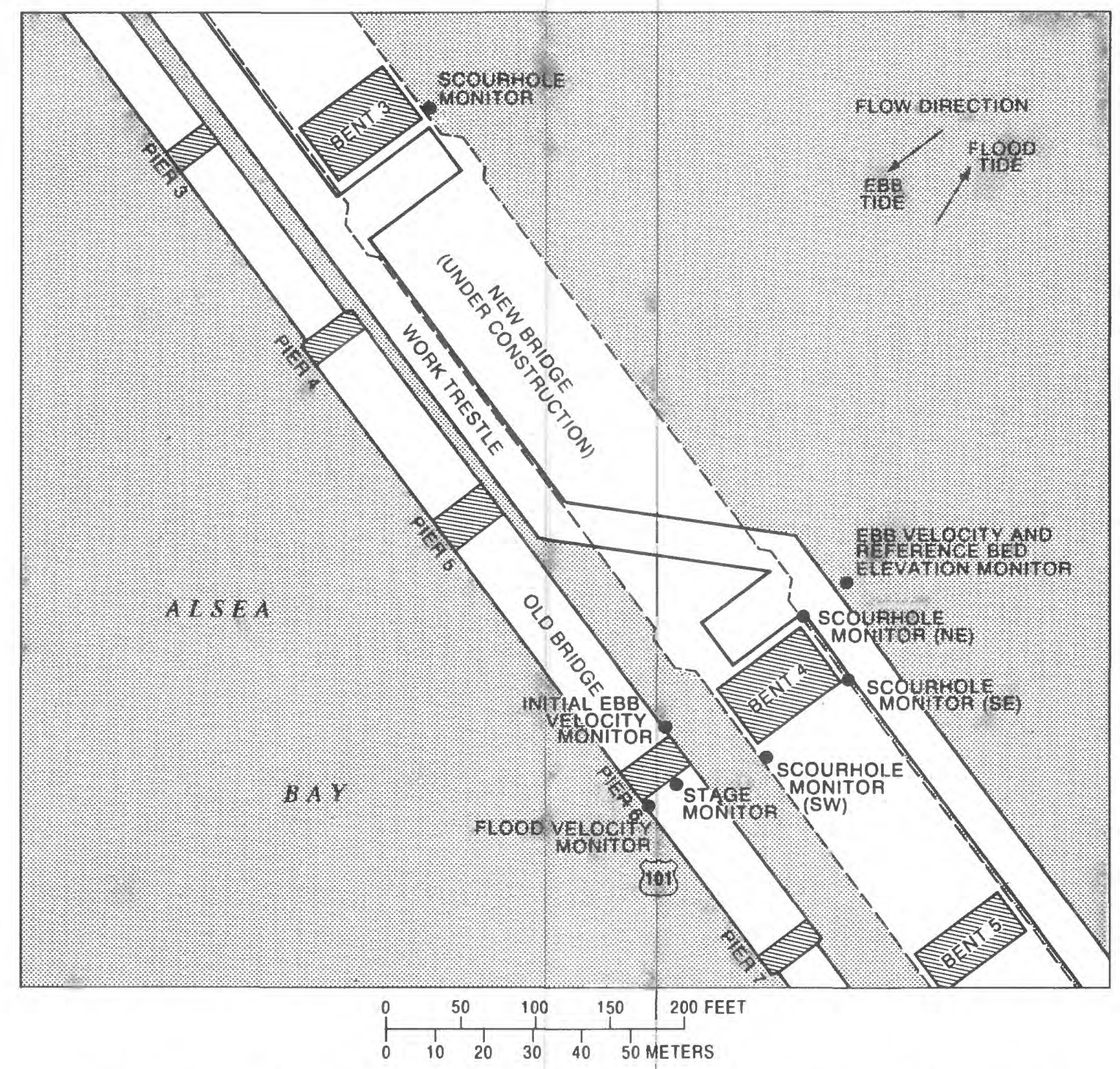

Figure 2.--Plan view of Highway 101 bridge, monitoring locations, and construction trestle at Alsea Bay, Oregon.

compared with a second set of data collected at bent 3 . Local scour at the southeast corner of bent 3 cofferdam was continuously monitored for 3 weeks, and scour at each corner was periodically measured from the time of cofferdam construction. Cross sections were surveyed from the mouth of the Alsea River to RM 12.5 up to the 100 -year-flood elevations so that maximum depths and velocities could be determined for estimating maximum local scour at the new bridge.

\section{Suspended-Sediment and Bed material}

Suspended-sediment and bed material samples were collected during maximum ebb-tide velocities at RM 5.0, in the vicinity of the new bridge (RM 1.1), and near the point where the Alsea River enter the Pacific Ocean. Suspended sediment samples were analyzed for particle size and concentration. Bed samples were analyzed for particle size. 


\section{METHODS OF DATA COLLECTION}

Water surface elevation, discharge, sediment, and bed material data were collected using USGS Techniques for Water Resources Investigations recommended procedures (Buchanan and Somers, 1974; Kennedy, 1983; Carter and Davidian, 1968; Guy and Norman, 1970). Cross-sectional data at the bridge and bed elevations that identified scour were collected using sonar equipment in conjunction with data-loggers. Techniques for use of this equipment were tested at the beginning of this project prior to data collection .

Cross-sectional data were collected by boat during high tide using electronic sonar instruments, with a transducer and data-logger. Depths obtained with the sonar device and corresponding times were recorded on a data-logger. Stationing was obtained by referencing the time the boat passed a known station, such as a bridge pier.

Bed-elevation measurements in scour holes and elevations of the streambed in the constricted zone were obtained by using a depth sounder interfaced to a data-logger, as used in the cross-section measurements, except the transducer was mounted to a fixed object (pier, or piling) and directed toward the scour hole or streambed. The depth sounder continuously monitored the same location and transferred the signal to a data-logger, which filtered signals and stored the data. During normal operation, the sonar depth sounder will measure depths within $\pm 0.5 \mathrm{ft}$, when scatter is minimum. Sonar equipment was subject to interference (scatter) when the signal path was obstructed by objects such as seaweed or sand particles. Scatter occurs when the sonic signal transmitted by the sonar device reflects off objects other than the intended streambed, such as when sand is suspended in the water column, or seaweed passes through the signal path. When scatter occurs, the data values are less than actual readings and can be detected and filtered. Mean daily scour-hole elevations were accurate within $\pm 1.5 \mathrm{ft}$ during periods when scatter was extreme, and within $\pm 0.5 \mathrm{ft}$ during periods when scatter was at a minimum.

Measurement of local scour using sonar was accomplished by mounting the tranducer near the scour hole and directing the signal toward the bottom of the hole. Determination of the deepest part of the scour hole was determined by field measurements. Measurements of bent 4, made on December 13, 1989, indicate that the deepest part of the southeast scour hole was at the corner, and the depth decreases $2 \mathrm{ft}$ for every $10 \mathrm{ft}$ of distance from the corner. Maximum depth at the southwest corner of bent 4 was located $2 \mathrm{ft}$ away from the corner and depth decreases about $1 \mathrm{ft}$ for every $10 \mathrm{ft}$ of distance from the corner. Directing the sonar transducers toward the deepest part of the scour holes at bent 4 was not difficult because these scour holes were large.

\section{DATA STORAGE}

Data collected for this study will be stored in the National Bridge Scour Program data base. The Bridge Scour Data Base System (BSDBS) is being developed by the USGS National Scour Study, in cooperation with the Federal Highway Administration. The BSDBS is an archival and retrieval system which also can be used to compute scour using various scour equations which are incorporated into the system. The BSDBS program is written in fortran and can be used on personal as well as mainframe computers, Landers (1991). Continuous and cross-sectional data collected during this project will be stored in BSDBS and are listed in tables 1 and 2.

\section{ILLUSTRATIONS AND TABLES}

Illustrations and tables that appear in the appendix part of this report are explained below. Bed elevations, gage height, and scour-hole elevation at bent 4 are shown in figures 3 and 4 . Scour-hole elevations at bent 3 are shown in figures 5 and 6. Cross sections taken in the vicinity of the Alsea Bay Highway 101 Bridge are shown in figures 7,8, and 9. Mean daily discharge for the Alsea River at Tidewater, during the period of study is shown in figure 10. Water-level correlations between Alsea River at Waldport, Alsea River at mouth of Drift Creek, and Alsea River at Kozy Kove during high and low flows are shown in figures 11 and 12. 
Daily scour-hole, bed-elevation data for bent 4 are listed in tables 3,4 , and 5 . Daily bed elevations in the vicinity of bent 4 are shown in table 6. Daily scour-hole bed-elevation data for bent 3 are listed in table 7. Maximum and minimum daily water levels for Alsea River at Waldport, Alsea River at mouth of Drift Creek, and Alsea River at Kozy Kove are listed in tables 8, 9d, and 10. Discharge measurements taken in the Alsea River Estuary during the period of study are listed in table 11.

Table 1.--Begin and end dates and accuracy of continuous 15-minute data that will be stored in the Bridge Scour Data Base System (Landers, 1991)

\begin{tabular}{ll|l}
\hline Begin date & End date & Accuracy \\
\hline
\end{tabular}

Ebb- and flood-tide velocity in the vicinity of bent 4

$\begin{array}{llr}\text { Apr. 16, 1989 } & \text { Apr. 20, 1989 } & \pm 0.25 \mathrm{ft} / \mathrm{s} \\ \text { Sept. 1, 1989 } & \text { Sept. 4, 1989 } & \pm .25 \mathrm{ft} / \mathrm{s} \\ \text { Dec. 14, 1989 } & \text { Dec. 18, 1989 } & \pm .25 \mathrm{ft} / \mathrm{s} \\ \text { Apr. 2, 1990 } & \text { Mar. 6, 1990 } & \pm .25 \mathrm{ft} / \mathrm{s}\end{array}$

Water-surface elevation at Waldport site, river mile 1.1

Feb. 26, 1988

Oct. 1, 1988

May 8, 1989

June 7, 1989

June 22, 1989

July 28, 1989

Sept. 9, 1989

Sept. 14, 1989

Sept. 23, 1989

Oct. 1, 1989

Nov. 1, 1989

Nov. 15, 1989

Dec. 11, 1989

Jan. 3, 1990

Feb. 14, 1990
Sept 30, 1988

May 7, 1989

June 6, 1989

June 19, 1989

July 2, 1989

Sept. 7, 1989

Sept. 11, 1989

Sept. 21, 1989

Sept. 30, 1989

Oct. 23, 1989

Nov. 14, 1989

Nov. 29, 1989

Jan. 1, 1990

Jan. 8, 1990

July 10, 1990

$$
\begin{aligned}
& \pm .25 \mathrm{ft} \\
& \pm .25 \mathrm{ft} \\
& \pm .25 \mathrm{ft} \\
& \pm .25 \mathrm{ft} \\
& \pm .25 \mathrm{ft} \\
& \pm .25 \mathrm{ft} \\
& \pm .25 \mathrm{ft} \\
& \pm .25 \mathrm{ft} \\
& \pm .25 \mathrm{ft} \\
& \pm .25 \mathrm{ft} \\
& \pm .25 \mathrm{ft} \\
& \pm .25 \mathrm{ft} \\
& \pm .25 \mathrm{ft} \\
& \pm .25 \mathrm{ft} \\
& \pm .25 \mathrm{ft}
\end{aligned}
$$

Water-surface elevation at confluence of Drift Creek, river mile 5.0
Apr. 19, 1989
Sept. 30, 1989
$\pm .25 \mathrm{ft}$
Oct. 1, 1989
July 11, 1990
$\pm .25 \mathrm{ft}$

Water-surface elevation at Alsea River at Kozy Kove, river mile 11.2

$\begin{array}{lll}\text { Apr. 21, } 1989 & \text { Sept. 30, 1990 } & \pm .75 \mathrm{ft} \\ \text { Oct. 1, 1989 } & \text { Feb. 18, 1990 } & \pm .75 \mathrm{ft} \\ \text { Feb. 28, 1990 } & \text { July 10, 1990 } & \pm .75 \mathrm{ft}\end{array}$


Table 1.--Begin and end dates and accuracy of continuous 15-minute data that will be stored in the Bridge Scour Data Base System --continued

Began date

End date

Apr. 3, 1989

May 25, 1989

Mar. 9, 1989

Apr. 12, 1989

Scour-hole elevations st S.E. corner of bent 4

June 14, 1989

Aug. 18, 1989

Oct. 1, 1989

Jan. 17, 1990

Jan. 31, 1990

Feb. 14, 1990

Scour-hole elevations at S.W. corner of bent 4

Apr. 13, 1989

July 28, 1989

Dec, 13, 1989

Jan. 17, 1990

Feb. 14, 1990
Aug. 14, 1989

Sept. 30, 1989

Jan. 10, 1990

Jan. 22, 1990

Feb. 12, 1990

May 1, 1990
July 24, 2989

Nov. 15,1989

Jan. 8, 1990

Feb. 9, 1990

July 9,1990

Accuracy

$6 \pm 1.5 \mathrm{ft}$

$\pm 1.5 \mathrm{ft}$

$$
\begin{aligned}
& \pm .25 \mathrm{ft} \\
& \pm .25 \mathrm{ft} \\
& \pm .25 \mathrm{ft} \\
& \pm .25 \mathrm{ft} \\
& \pm .25 \mathrm{ft} \\
& \pm .25 \mathrm{ft}
\end{aligned}
$$

$\pm .25 \mathrm{ft}$

$\pm .25 \mathrm{ft}$

$\pm .25 \mathrm{ft}$

$\pm .25 \mathrm{ft}$

$\pm .25 \mathrm{ft}$

Scour-hole elevations at S.E. corner of bent 3

May 3, 1990

May 23, 1990

$\pm .25 \mathrm{ft}$

Reference-bed elevation at bent 4

Apr. 13, 1989

Mar. 23, 1990

$\pm .25 \mathrm{ft}$ 
Table 2.--Dates for cross-sectional measurements of the Alsea River at Waldport, Oregon. Distances shown are referenced to the center of the old Highway 101 Bridge

\begin{tabular}{|c|c|c|c|c|c|c|c|c|c|c|c|}
\hline & & & & & & Date & & & & & \\
\hline Station & $8 / 88$ & $9 / 88$ & $11 / 88$ & $12 / 88$ & $1 / 89$ & $2 / 89$ & $3 / 89$ & $4 / 89$ & $5 / 89$ & $10 / 89$ & $3 / 90$ \\
\hline $600 \mathrm{ft}$ west & $x$ & $x$ & $x$ & & $x$ & & $x$ & $x$ & $x$ & $x$ & $x$ \\
\hline $300 \mathrm{ft}$ west & $x$ & $x$ & $x$ & & $x$ & & & $x$ & $x$ & $x$ & $x$ \\
\hline $100 \mathrm{ft}$ west & $x$ & $x$ & $x$ & $x$ & $x$ & & $\mathrm{x}$ & $x$ & & & \\
\hline $50 \mathrm{ft}$ west & $x$ & $x$ & $x$ & $x$ & $x$ & $x$ & $\mathrm{x}$ & $x$ & $x$ & $x$ & $x$ \\
\hline $50 \mathrm{ft}$ east & $x$ & $x$ & $x$ & $x$ & $x$ & & $x$ & $x$ & $x$ & $x$ & $x$ \\
\hline $100 \mathrm{ft}$ east & $x$ & $x$ & $x$ & $x$ & & & & & & & \\
\hline $200 \mathrm{ft}$ east & & & & & & & $x$ & $x$ & $x$ & & \\
\hline $300 \mathrm{ft}$ east & $x$ & $x$ & $x$ & $x$ & $x$ & & & $x$ & $x$ & $x$ & $x$ \\
\hline $600 \mathrm{ft}$ east & $x$ & $x$ & $x$ & & $x$ & & $x$ & $x$ & $x$ & $x$ & \\
\hline
\end{tabular}




\section{SELECTED REFERENCES}

Buchanan T.J., and Somers W.P., 1974, Stage measurement at gaging stations: U.S. Geological Survey Techniques of Water-Resources Investigations, book 3, chapter A7, 28 p.

Carter, R.W., and Davidian J., 1968, General procedure for gaging streams: U.S. Geological Survey Techniques of Water-Resources Investigations, book 3, chapter A6, 13p.

Guy H.P., and Norman V.W., 1970, Field methods for measurement of fluvial sediment: U.S. Geological Survey Techniques of Water-Resources Investigations, book 3, chapter C2, 59 p.

Jarrett, R.D., and Boyle J.M., 1986, Pilot study for collection of bridge-scour data: U.S. Geological Survey Water Resources Investigations Report 86-4030, 46 p.

Kennedy. E.J., 1983, Computation of continuous records of streamflow: U.S. Geological Survey Techniques of Water-Resources Investigations, Book 3, Chapter A13, 53 p.

Landers M.N., 1991, Bridge scour data base system: Las Vegas, Nevada, Fifth Interagency Sedimentation Conference, 1991, proceedings, p. 2-121:2-126 


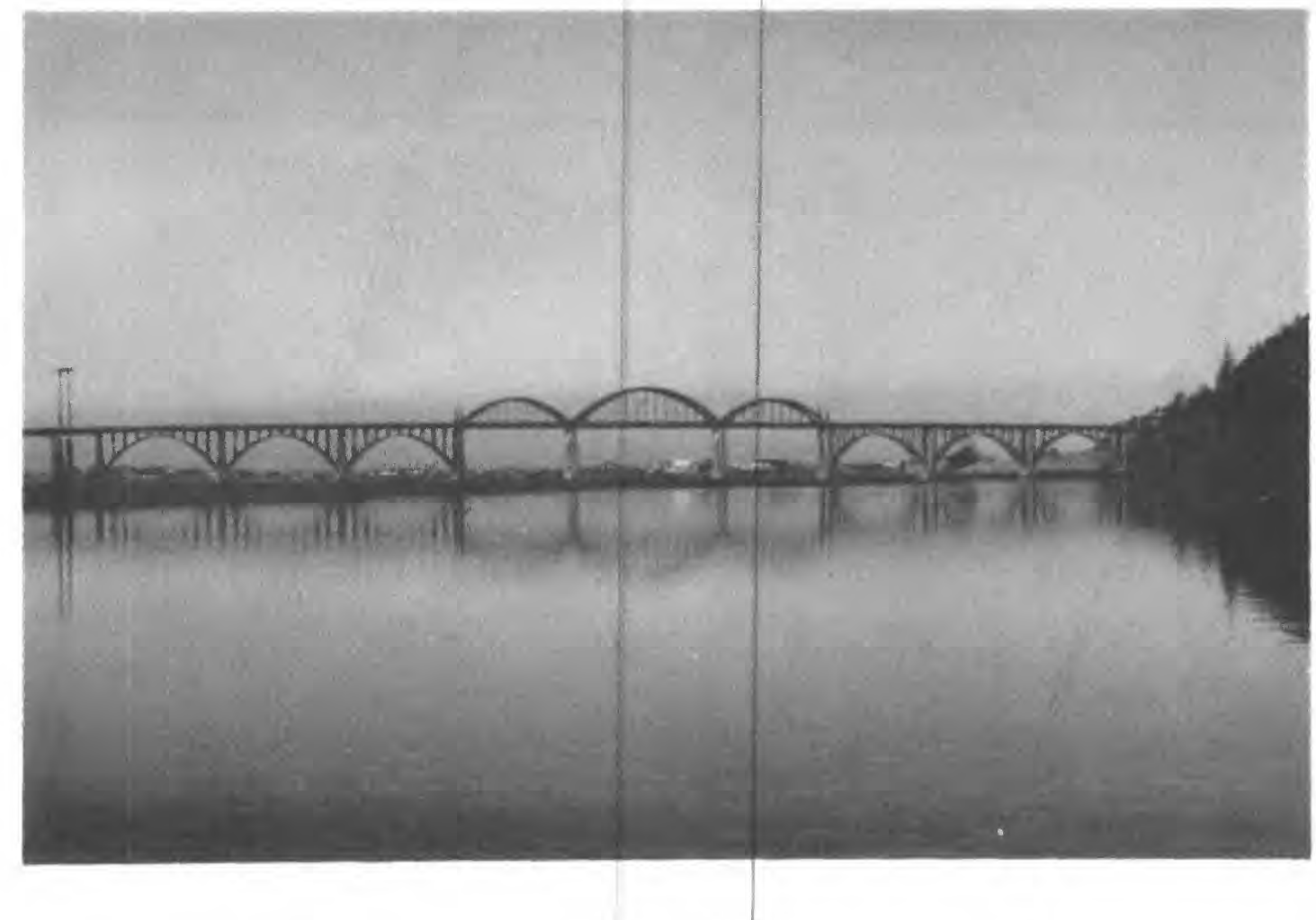

Old Highway 101 Alsea Bay Bridge at Waldport, Oregon. Photo taken before construction of new bridge began.

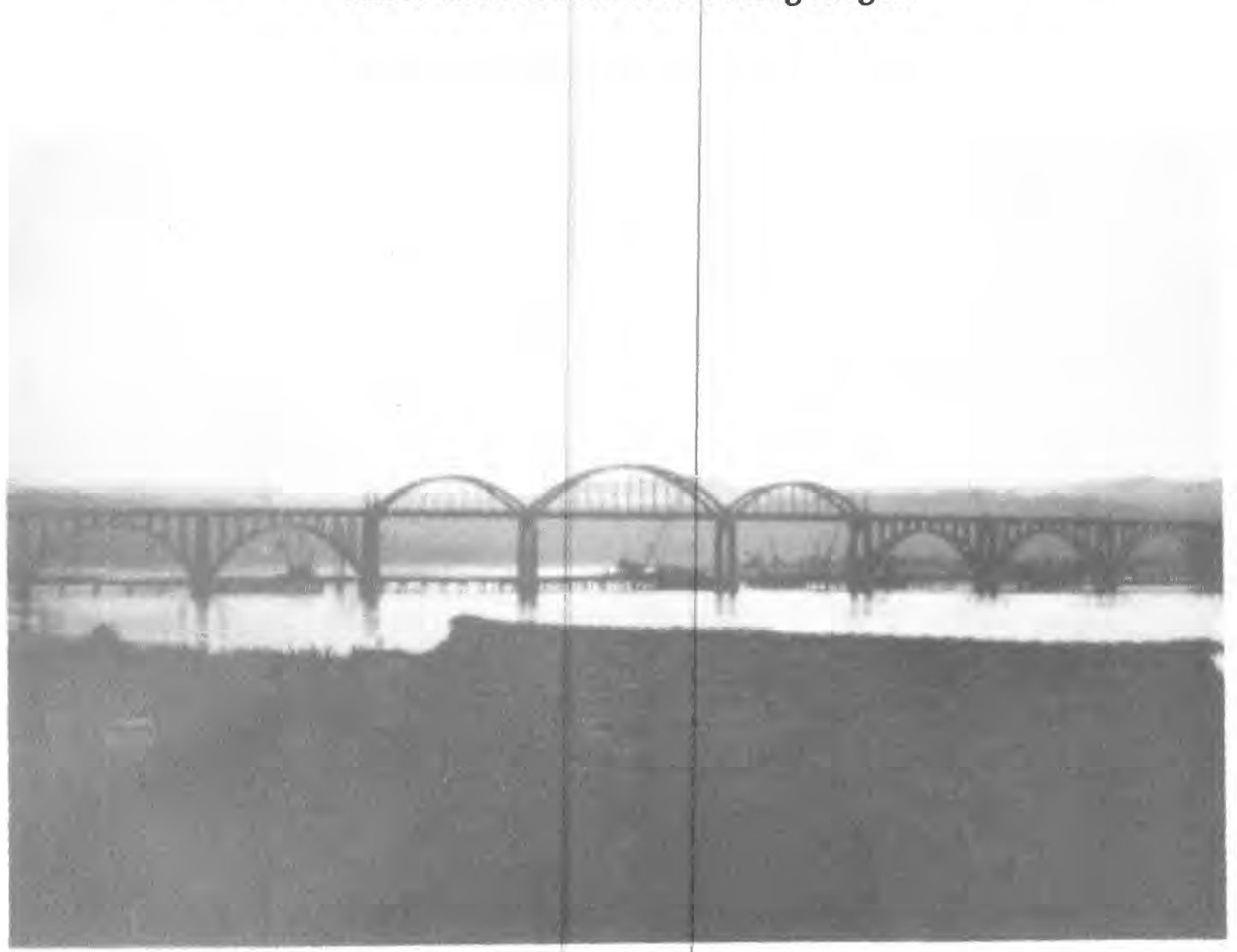

Old Highway 101 Alsea Bay Bridge at Waldport, Oregon. Photo taken after construction of new bridge started. Work trestle is visible near bridge. 


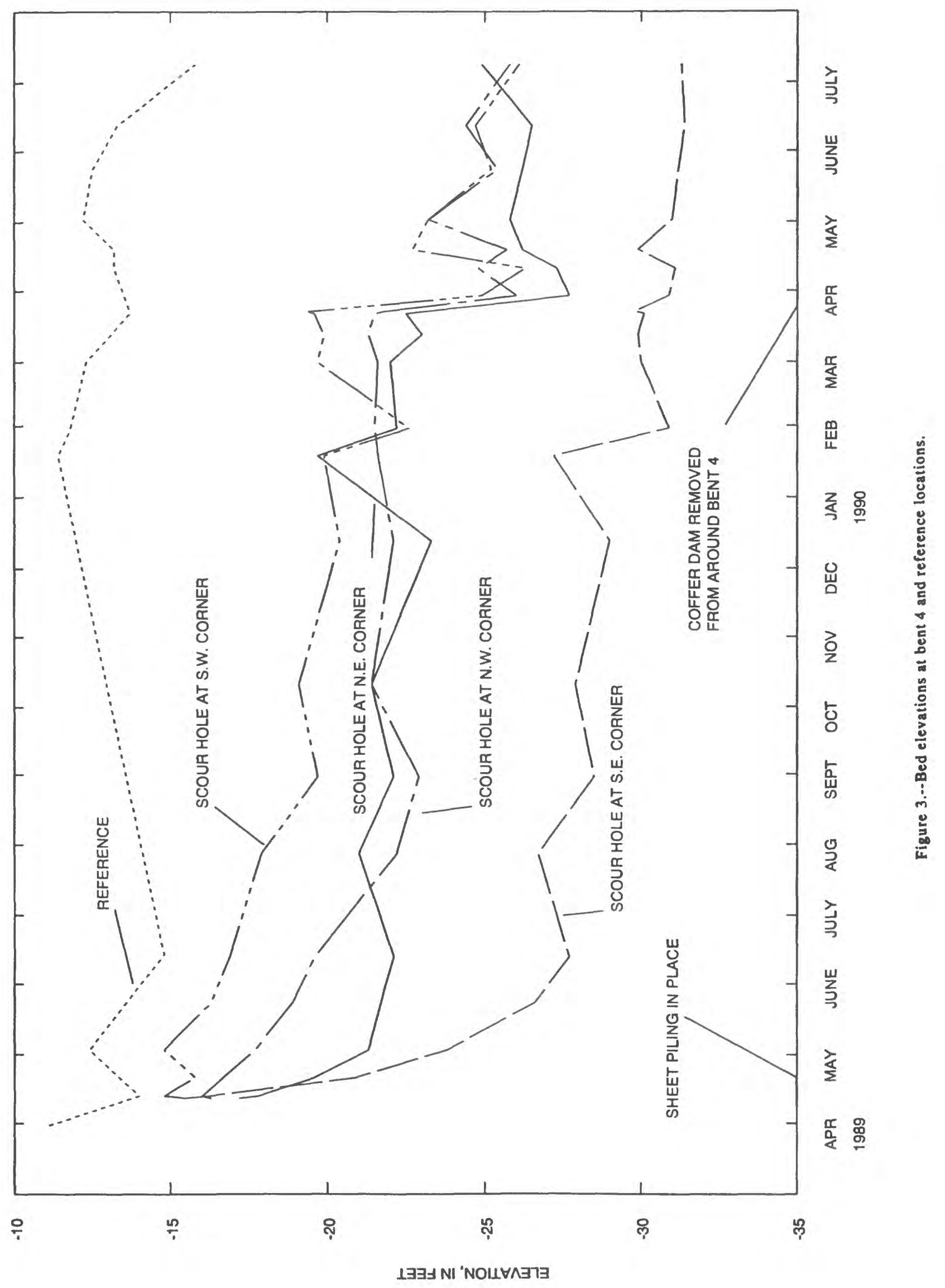




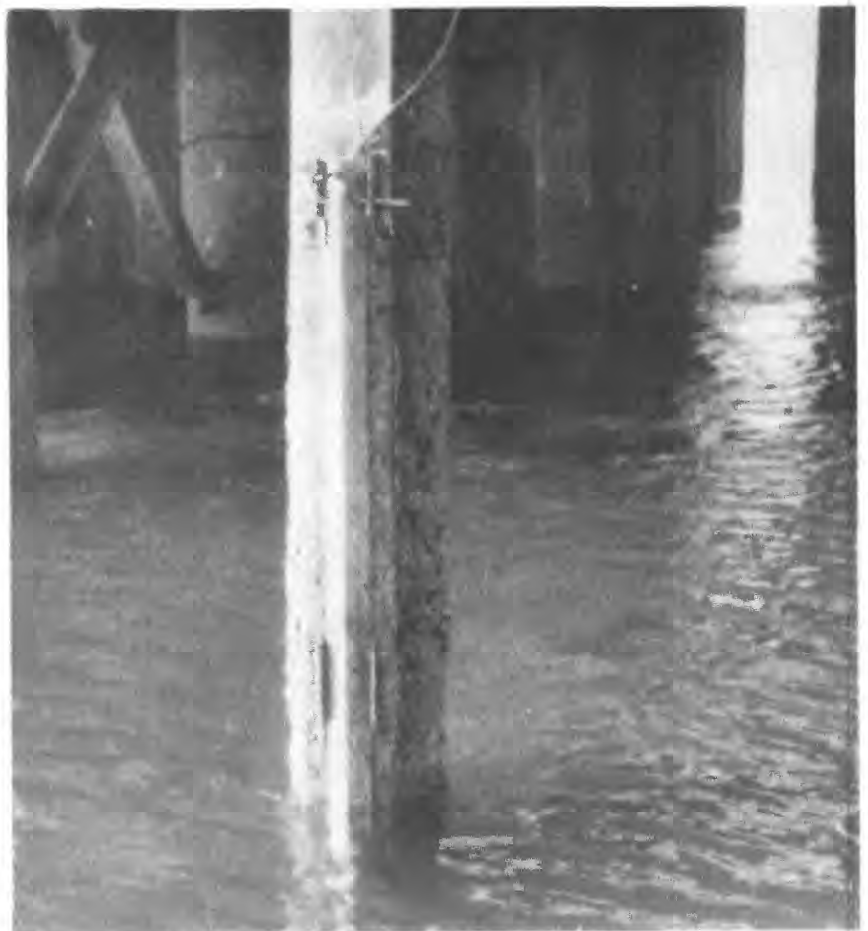

Pile and mount for depth sounder and velocity sensor. Sensors are beneath water surface, in monitoring position.

Velocity sensor and depth transducer raised for cleaning. 

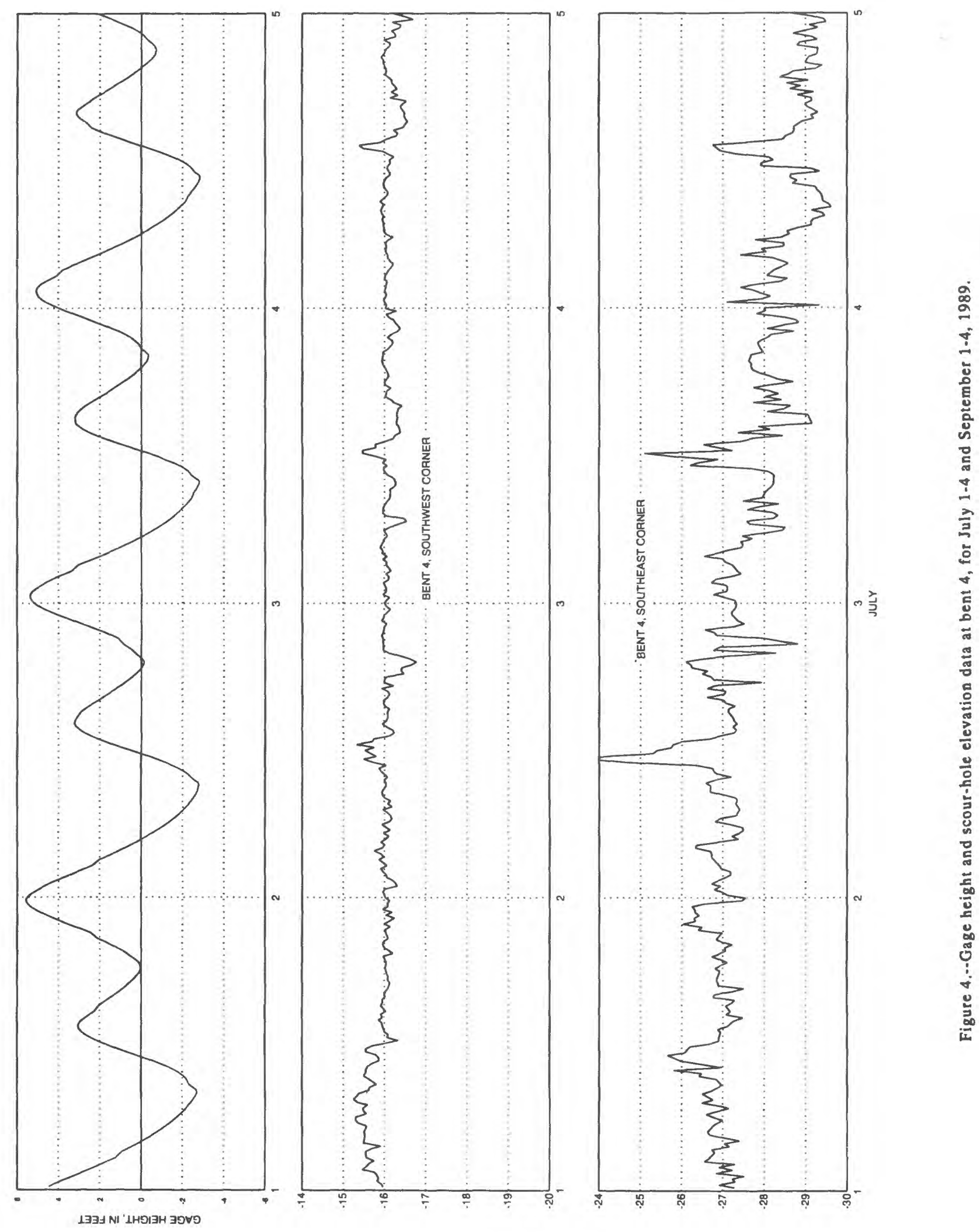

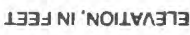



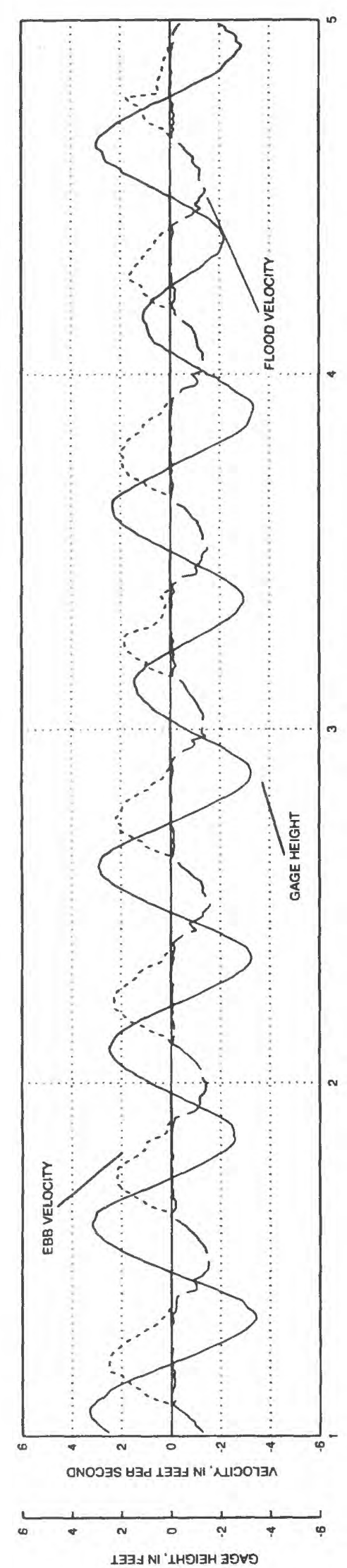
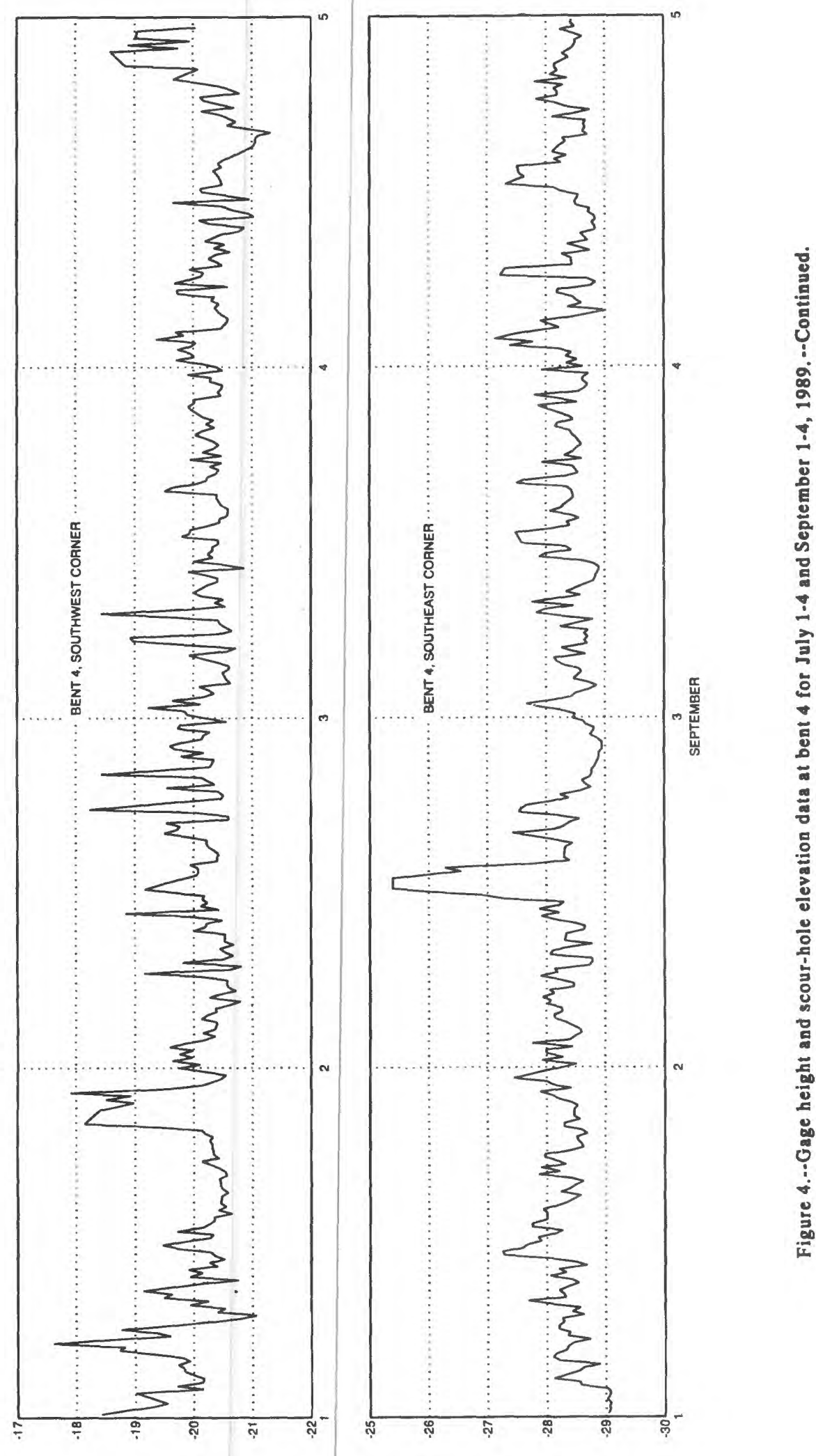

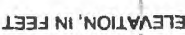




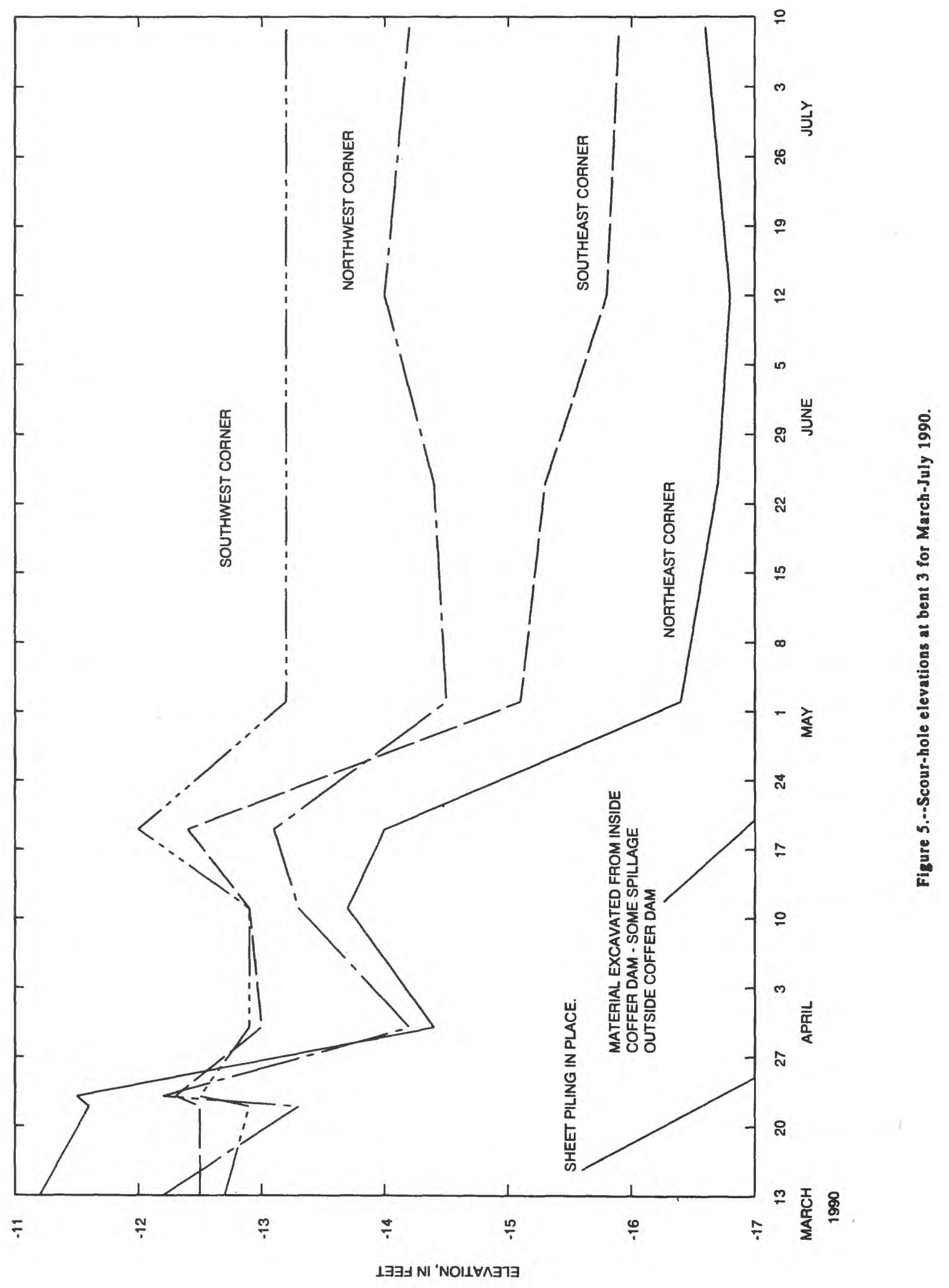




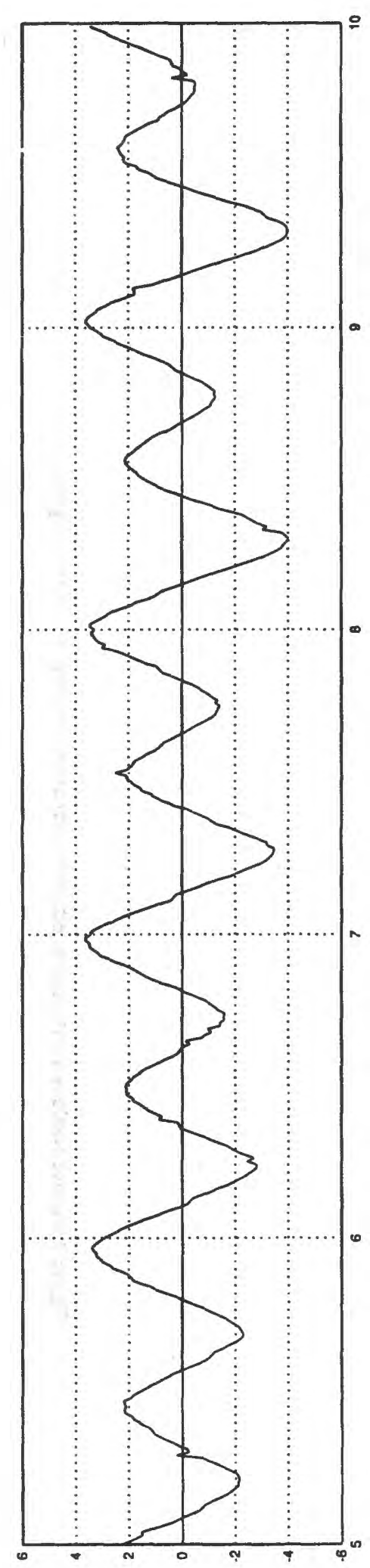

$1 \exists \exists+\mathrm{NI}$ ' 1 Hอเ

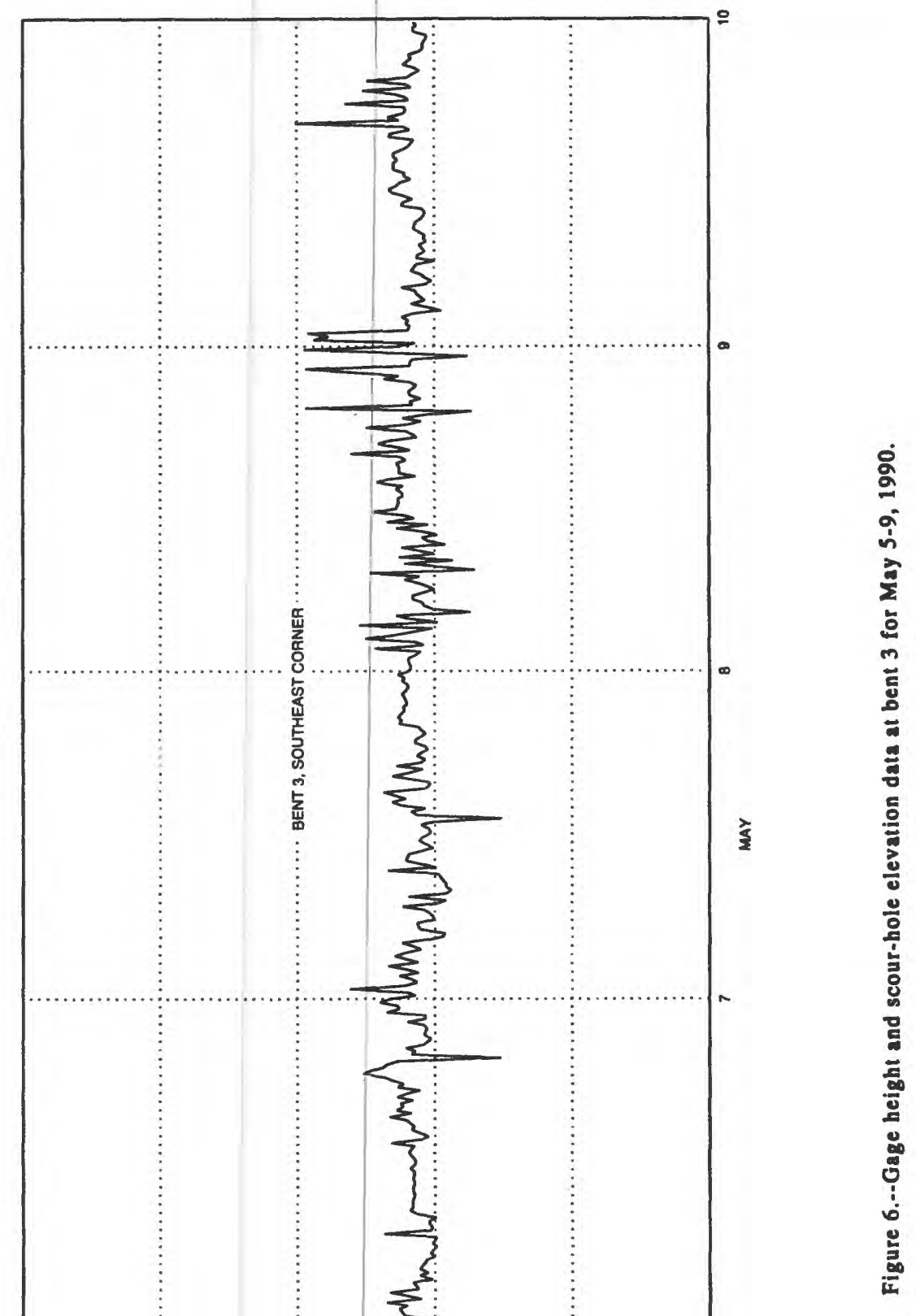




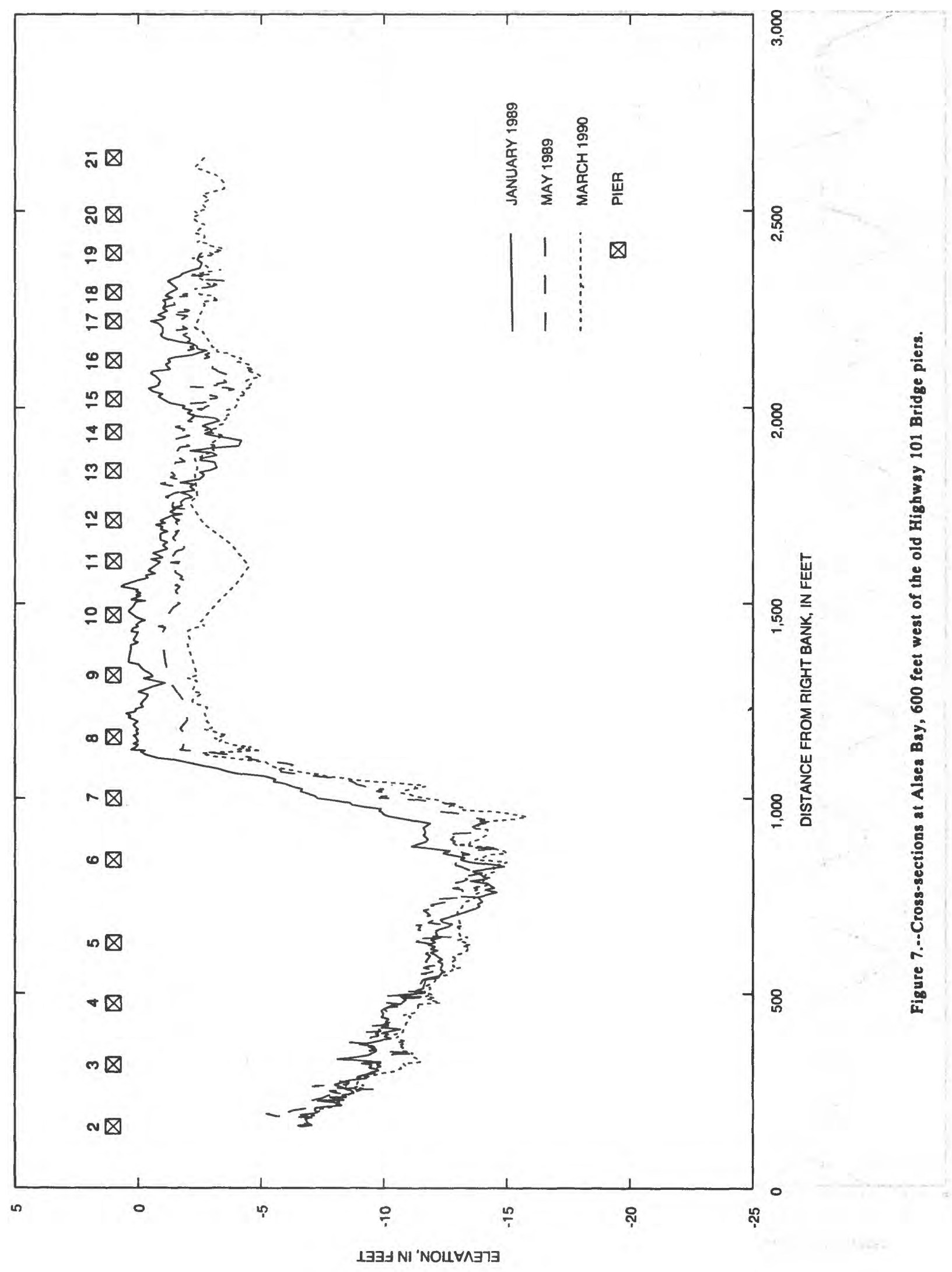




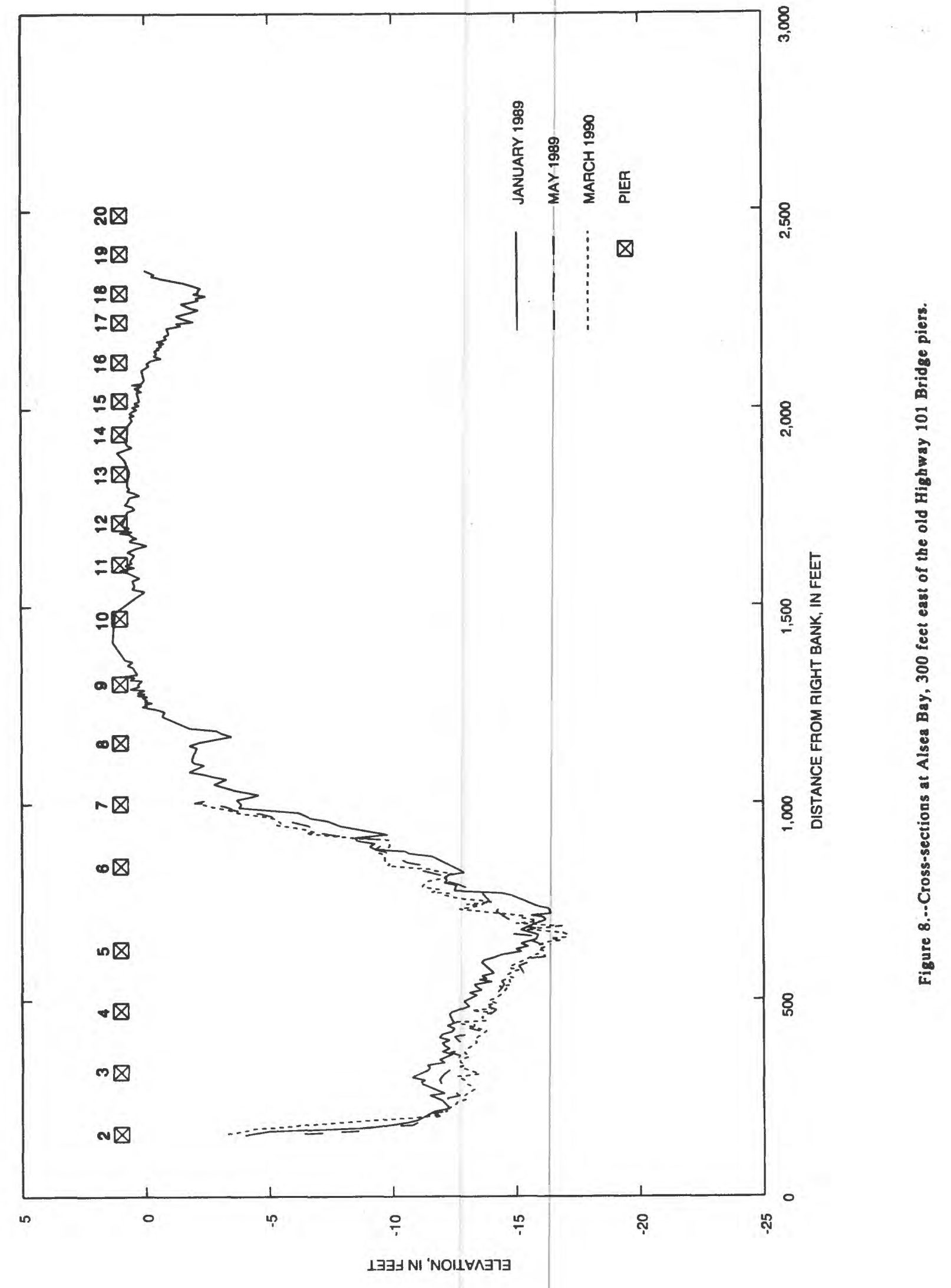




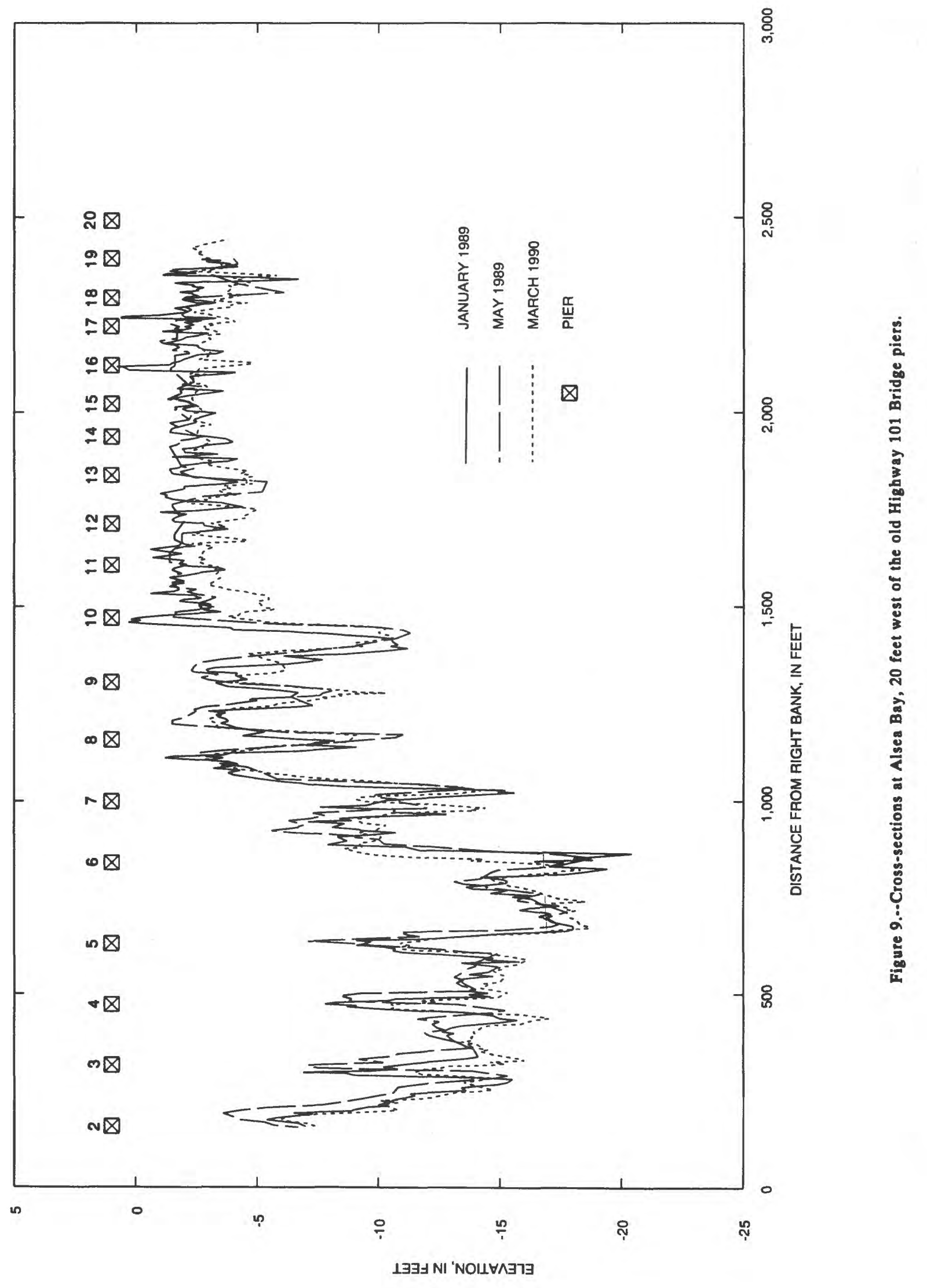




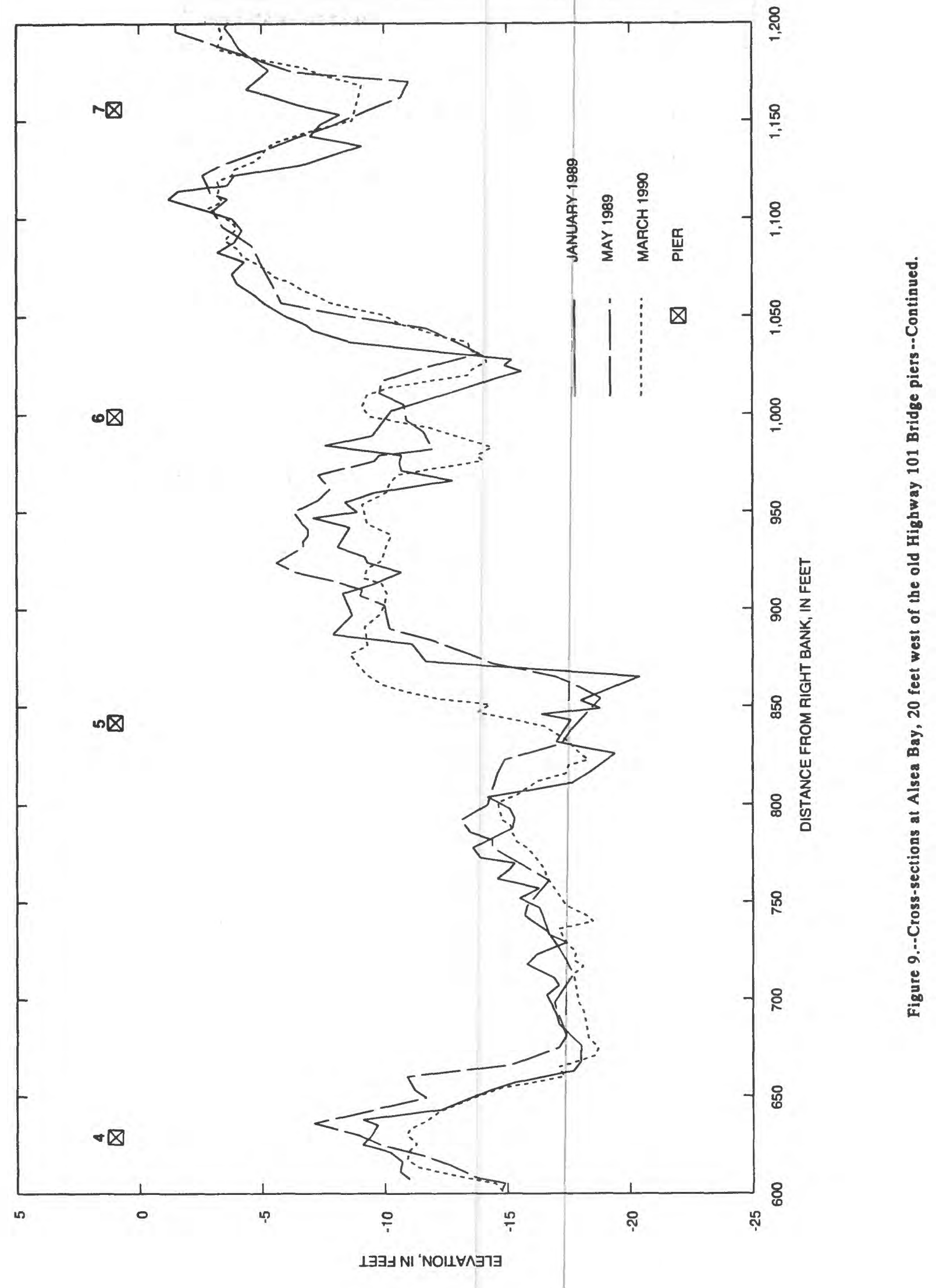



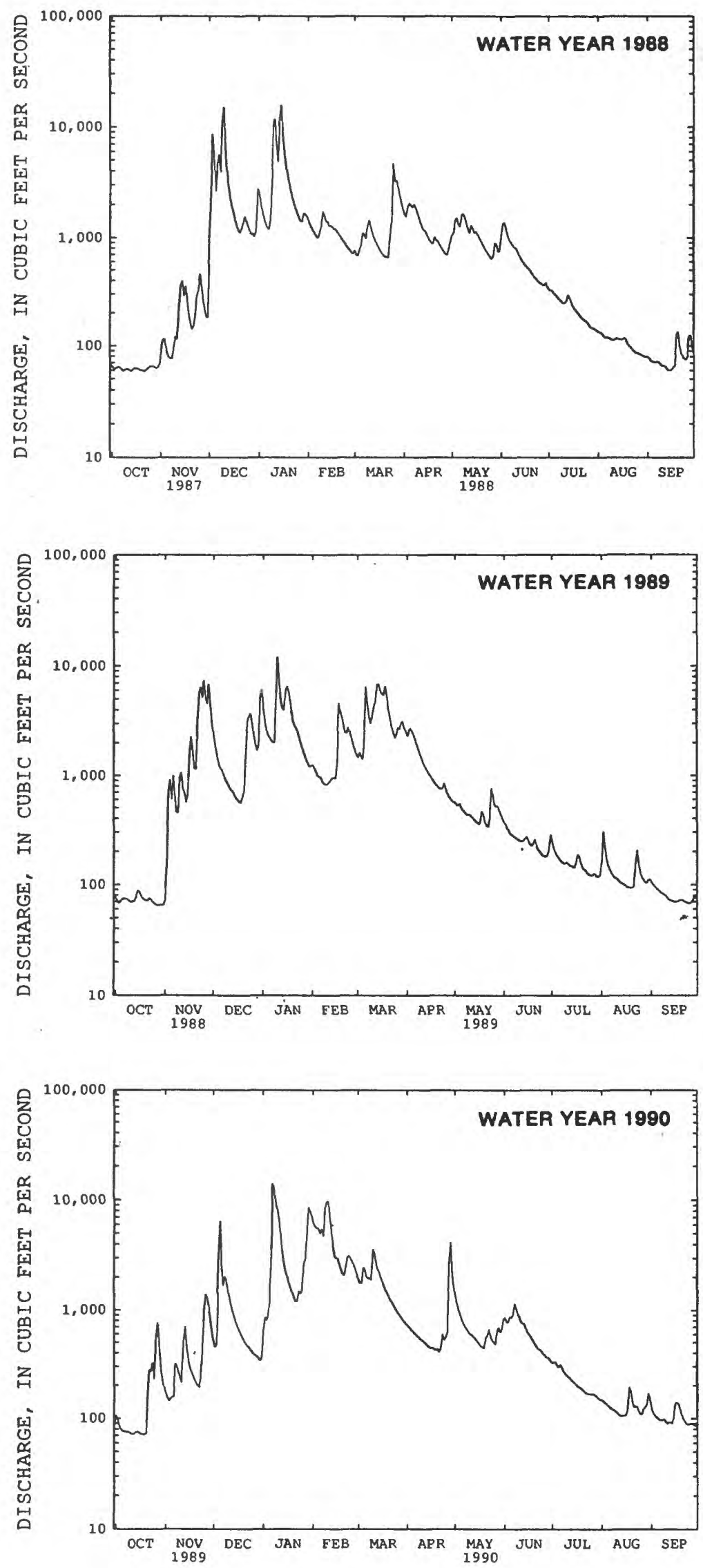

Figure 10.--Mean daily discharge for Alsea River near Tidewater for water years 1988-90. 


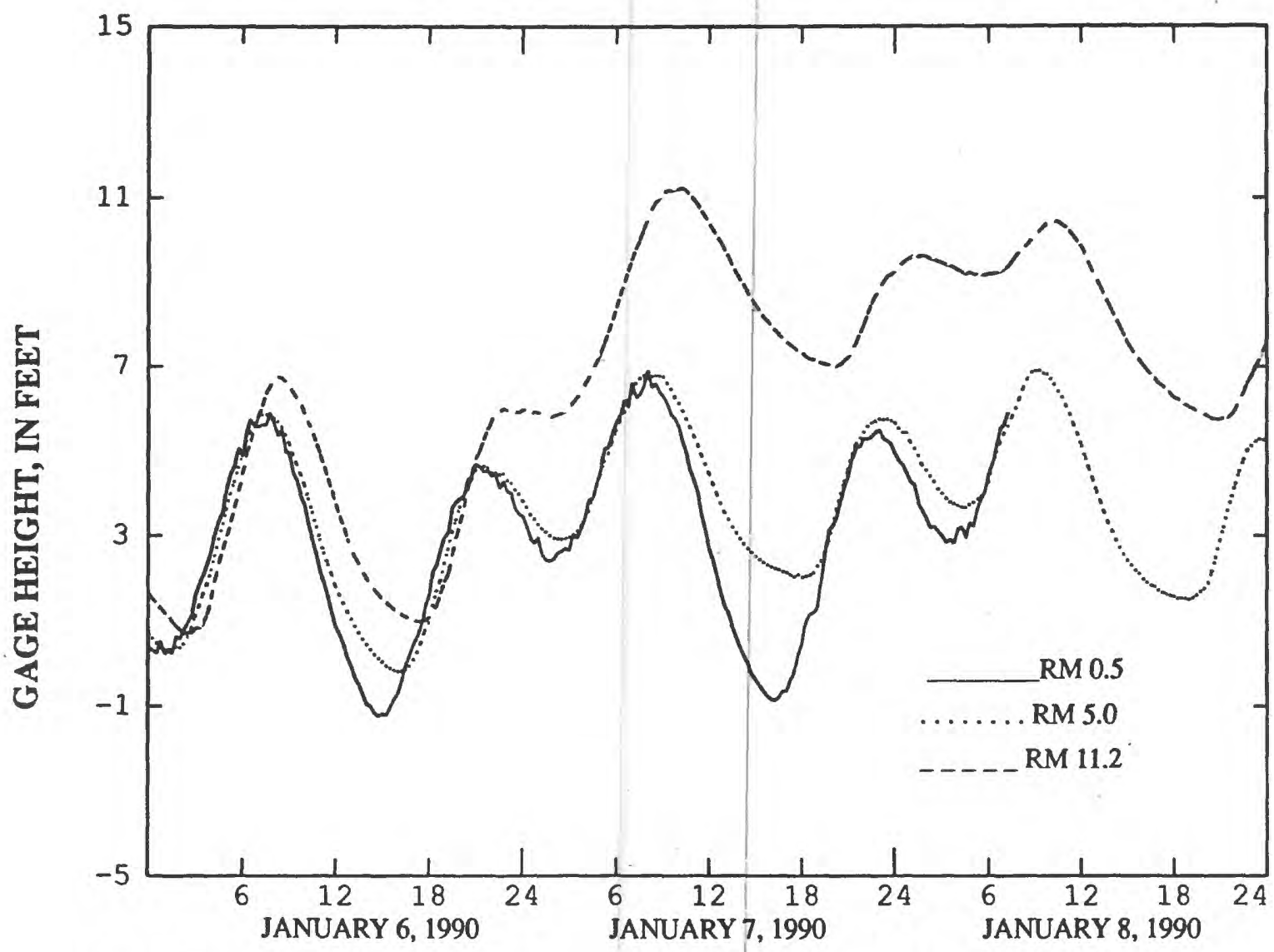

Figure 11..-Water levels during bigh flow of the Alsea River at Waldport (RM 0.5), Drift Creek (RM 5.0), and Kozy Kove (RM 11.2) for January 6-8, 1990.

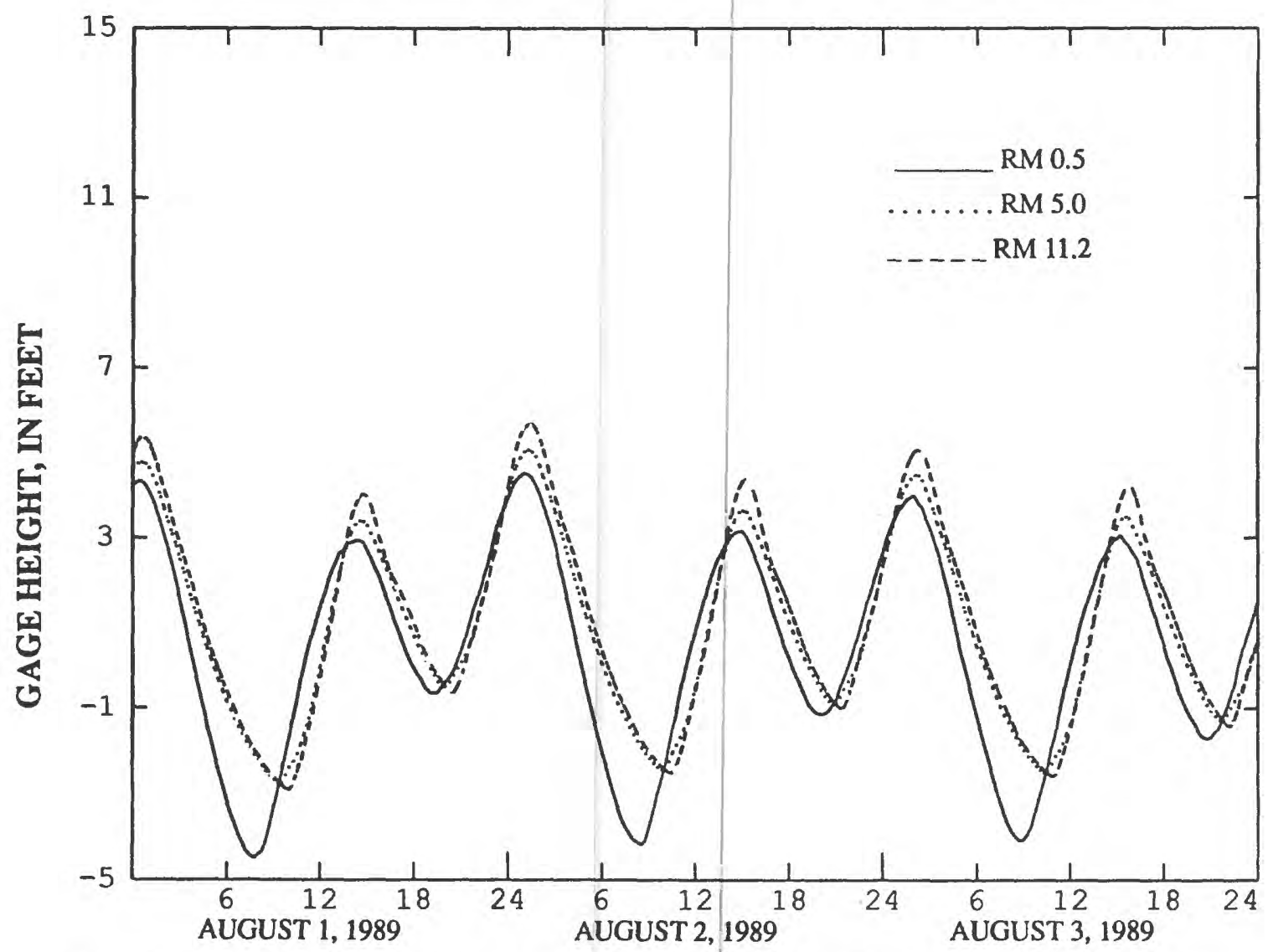

Figure 12.--Water levels during low flow of the Alsea River at Waldport (RM 0.5), Drift Creek (RM 5.0), and Kozy Kove (RM 11.2) for August 1-3, 1989. 


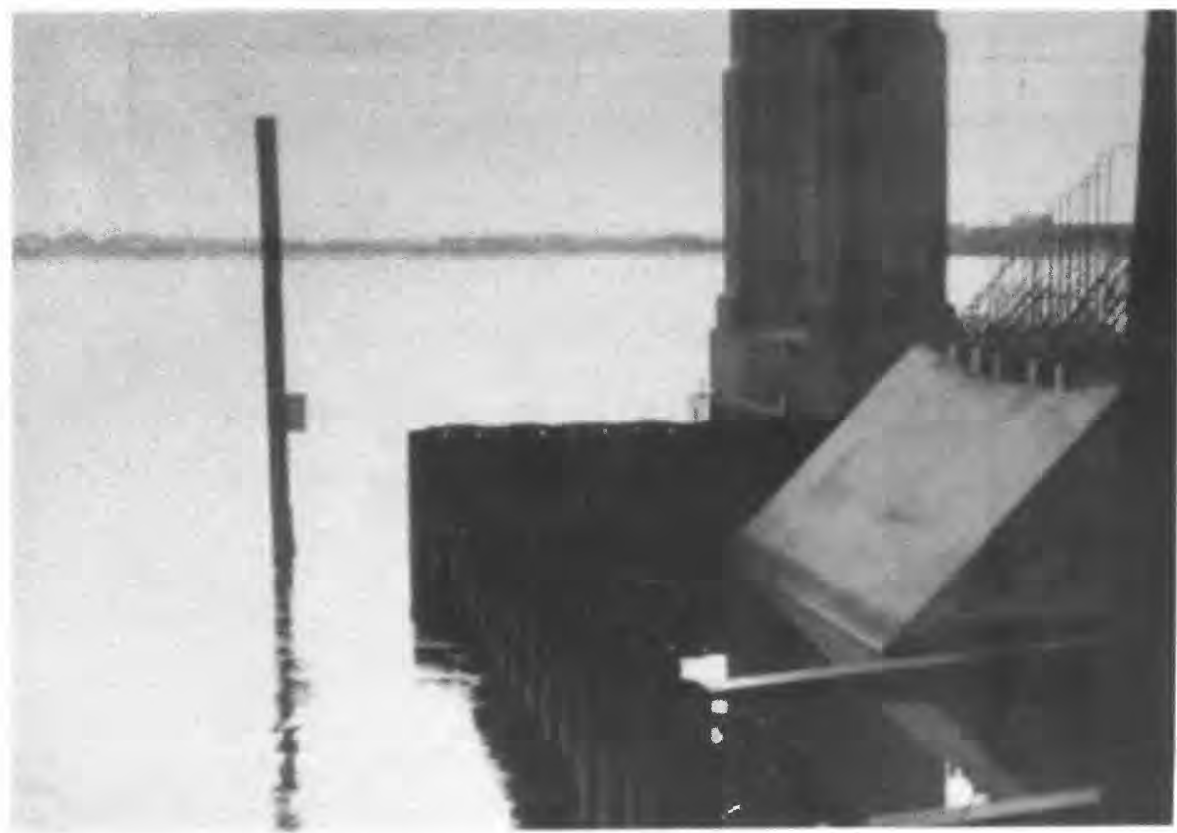

Looking west at bent 4 coffer dam (new Highway 101 bridge under construction) and old highway pier 6. Pile with shelter located left of bent 4 houses depth sounding equipment used to monitor scour hole depths at the southwest corner of bent 4 .

Stilling well and shelter at Alsea River at Kozy Kove, RM 11.2.

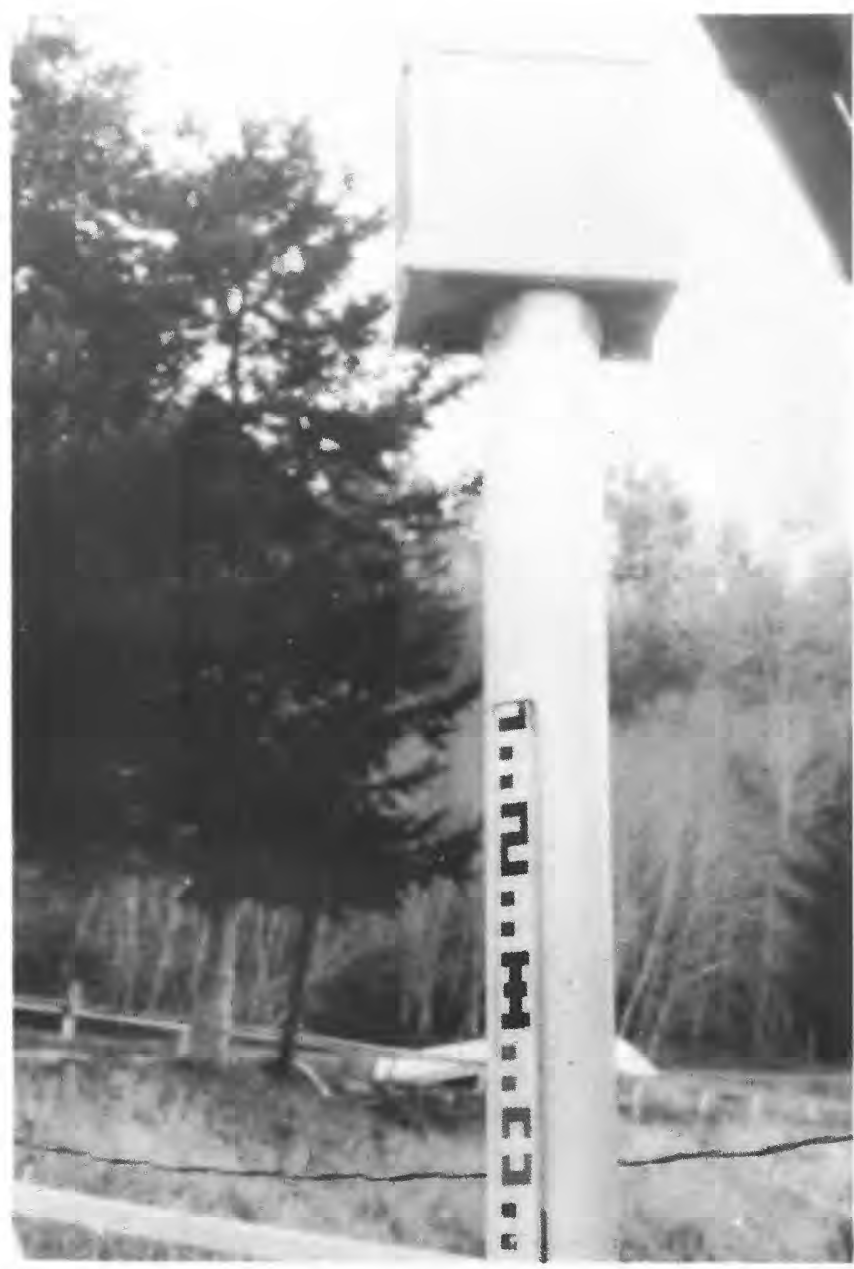


LOCATION.--Lat $44^{\circ} 25^{\prime} 58^{\prime \prime}$, long $124^{\circ} 04^{\prime} 14^{\prime \prime}$, in SE $1 / 4$ SW $1 / 4$ sec.18, T.13 S., R.11 W., Lincoln County, Hydrologic Unit 17100205; gage on a metal pile supporting the work trestle, 10 feet northeast of bent 4 of new Highway 101 Bridge, 0.5 miles north of พaldport.

PERIOD OF RECORD.--March 1989 to May 1989 (scour-hole elevation) - discontinued.

GAGE.--Bed-elevation recorder. Sonic depth sounder in conjunction with a data logger. Datum of gage is 0.00 feet above National Geodetic vertical Datum of 1929 (levels by Oregon Department of Transportation).

REMARKS.--Bed elevations at this site indicate local scour from ebb tide at the northeast corner of bent 4 cofferdam of the new Highway 101 Bridge.

EXTREMES FOR PERIOD OF RECORD.--Maximum daily recorded: -16.1 feet on April 2, 1989 ; minumum daily recorded: -22.3 feet on May 16,1989 .

DAILY ELEVATION, IN FEET, WATER YEAR OCTOBER 1988 TO SEPTEMBER 1989

MARCH APRIL

DAY RECORDED OBSERVED RECORDED OBSERVED
MAY

RECORDED OBSERVED

\begin{tabular}{|c|c|c|c|c|c|c|}
\hline 1 & -- & -- & -16.2 & --- & -20.7 & -- \\
\hline 2 & -- & -- & -16.1 & -- & -20.6 & $\cdots$ \\
\hline 3 & --- & -- & -16.3 & $\ldots$ & -20.8 & -21.3 \\
\hline 4 & -- & -- & -- & -- & -21.3 & -- \\
\hline 5 & --- & -- & --- & -- & -21.3 & -- \\
\hline 6 & -- & -- & -- & - & -21.3 & $-\infty$ \\
\hline 7 & -- & -- & -- & - & -21.3 & -- \\
\hline 8 & -- & -- & -- & - & -21.1 & -- \\
\hline 9 & -16.7 & -- & -- & - & -21.2 & -- \\
\hline 10 & -17.2 & -- & -- & - & -21.2 & -- \\
\hline 11 & -17.1 & - - & --- & -17.9 & -21.1 & -- \\
\hline 12 & -17.1 & -- & -- & -17.2 & -21.4 & -- \\
\hline 13 & -17.3 & -- & -18.3 & -17.8 & -21.8 & -- \\
\hline 14 & -17.4 & -- & -18.3 & - & -21.2 & --- \\
\hline 15 & -17.4 & --- & -19.0 & - & -21.2 & -- \\
\hline 16 & -17.2 & -- & -18.7 & - & -22.3 & --- \\
\hline 17 & -17.1 & -- & -19.0 & -- & -21.5 & --- \\
\hline 18 & -17.2 & -- & -18.1 & - & -21.4 & -- \\
\hline 19 & -17.0 & -- & -17.6 & - & -21.7 & --- \\
\hline 20 & -16.7 & -- & -19.0 & & -21.8 & -- \\
\hline 21 & -16.7 & -- & -19.5 & -19.6 & -21.3 & --- \\
\hline 22 & -16.9 & -- & -19.6 & $--i$ & -21.3 & --- \\
\hline 23 & -16.9 & -- & -19.9 & $\ldots$ & -21.4 & --- \\
\hline 24 & -16.9 & -- & -20.3 & - & -21.7 & -21.7 \\
\hline 25 & -17.0 & -- & -20.4 & & -20.7 & --- \\
\hline 26 & -16.9 & -- & -20.2 & - & -- & --- \\
\hline 27 & -17.0 & -- & -20.5 & -- & -- & -- \\
\hline 28 & -17.0 & -- & -20.5 & - & -- & --- \\
\hline 29 & -16.9 & -- & -20.4 & - & -- & --- \\
\hline 30 & -16.6 & -- & -20.4 & & --- & --- \\
\hline 31 & -16.3 & -- & -- & & -- & -- \\
\hline
\end{tabular}


Table 4.--Scour-hole bed elevation for southeast corner of bent 4, Alsea River at Waldport

LOCATION.--Lat $44^{\circ} 25^{\circ} 58^{\prime \prime}$, long $124^{\circ} 04^{\prime} 14^{\prime \prime}$, in SE $1 / 4$ SW $1 / 4$ sec.18, T.13 S., R.11 W., Lincoln County, Hydrologic Unit 17100205; gage on metal pile supporting the work trestle 10 feet southeast of bent 4 of new Highway 101 Bridge, 0.5 miles north of waldport.

PERIOD OF RECORD.--June 1989 to April 1990 scour-hole elevation).

GAGE.--Bed-elevation recorder. Sonic-depth sounder in conjunction with a data logger. Datum of gage is 0.00 feet above National Geodetic Vertical Datum of 1929 (levels by oregon Department of Transportation).

REMARKS.--Bed elevations at this site indicate local scour from ebb tide at the southeast corner of bent 4 cofferdam of the new Highway 101 Bridge.

EXTREMES FOR PERIOD OF RECORD.--Maximum elevation -26.7 feet on June 16, 17, 19, 20, 1989; minimum elevation, -31.7 feet on February 9, 1990.

DAILY SCOUR-HOLE ELEVATIONS, FEET, WATER YEAR OCTOBER 1988 TO SEPTEMBER 1989

APRIL MAY JUNE JULY AUGUST SEPTEMBER DAY RECORDED OBSERVED RECORDED OBSERVED RECORDED OBSERVED RECORDED OBSERVED RECORDED OBSERVED RECORDED OBSERVED

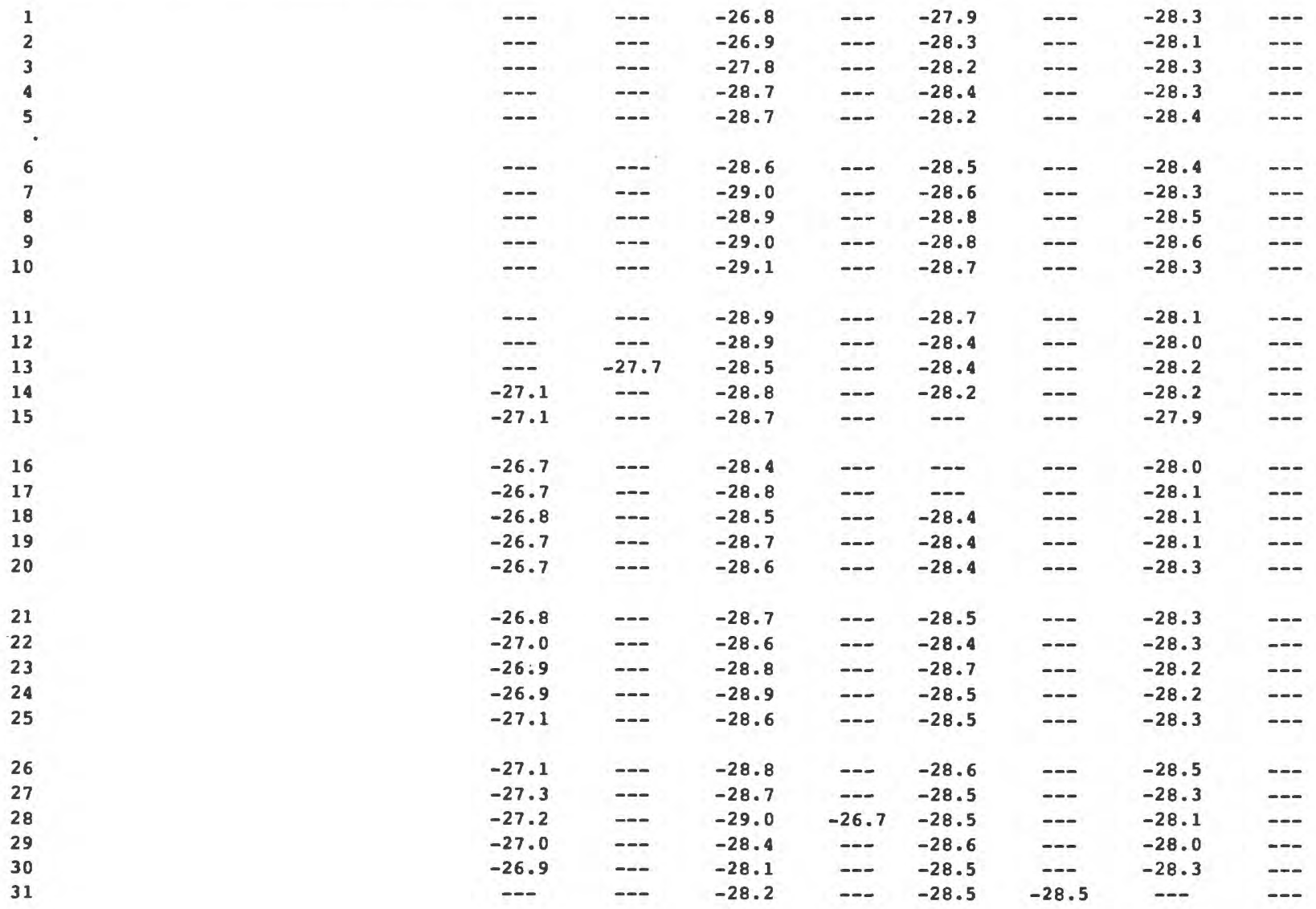


Table 4.--Scour-hole bed elevations for southeast corner of bent 4, Alsea River at waldport--Continued

DAILY SCOUR-HOLE ELEVATIONS, FEET, WATER YEAR OCTOBER 1989 TO SEPTEMBER 1990

OCTOBER NOVEMBER DECEMBER JANUARY FEBRUARY MARCH DAY RECORDED OBSERVED RECORDED OBSERVED RECORDED OBSERVED RECORDED OBSERVED RECORDED OBSERVED RECORDED OBSERVED

\begin{tabular}{|c|c|c|c|c|c|c|c|c|c|c|c|}
\hline-28.1 & -- & -28.4 & --- & -28.7 & -- & -29.1 & -- & -30.6 & -. & -30.7 & -- \\
\hline-28.3 & -- & -28.3 & -- & -28.9 & -- & -28 & -- & -30.8 & --- & -30.4 & -- \\
\hline-28.2 & --- & -28.4 & --- & -28.6 & -- & -28.9 & --- & -31.3 & -- & -30.3 & -- \\
\hline-28.4 & -- & -28.6 & -- & -29.1 & -- & -28.7 & -- & -31.2 & -- & -30.3 & --- \\
\hline-28.5 & --- & -28.7 & --- & -29.0 & -- & -29.0 & -- & -30.7 & --- & -30.3 & --- \\
\hline-28.5 & --- & -28.5 & -- & -29.0 & -- & -29.0 & -- & -31.2 & -- & -30.3 & -- \\
\hline-28.4 & --- & -28.7 & -- & -28.9 & -- & -29.3 & $-\infty$ & -30.1 & -- & -30.3 & --- \\
\hline-28.5 & --- & -28.7 & --- & -28.5 & -- & -29.9 & -- & -31.2 & -- & -30.2 & --- \\
\hline$-28 \cdot 6$ & --- & -28.5 & -- & -28.7 & -- & -29.2 & -.- & -31.7 & -- & -30.2 & --- \\
\hline-28.6 & --- & -28.8 & -- & -28.6 & -- & --- & -- & -31.6 & -- & -30.3 & --- \\
\hline-28.5 & -27.9 & -28.7 & -- & -28.8 & -- & --- & -- & -30.8 & -- & $-30 \cdot .5$ & --- \\
\hline-27.9 & $-=-$ & -28.6 & --- & -28.6 & --- & --- & -- & --- & --- & -30.6 & --- \\
\hline-28.2 & --- & -28.9 & -- & -28.6 & -29.0 & -- & -- & --- & --- & -30.2 & -29.9 \\
\hline-28.1 & --- & -28.7 & --- & -28.8 & 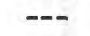 & --- & -- & -31.2 & --- & -30.3 & --- \\
\hline-28.1 & --- & -29.1 & -- & -28.8 & $\cdots$ & $-\cdots$ & -- & -29.7 & --- & -30.5 & --- \\
\hline$-28 \cdot 3$ & -- & -28.7 & --- & -28.9 & --- & -- & -- & -30.2 & --- & -30.4 & --- \\
\hline-28.2 & --- & -28.8 & --- & -28.5 & --- & -- & -- & -29.9 & --- & -30.4 & -- \\
\hline-28.3 & --- & -28.6 & -- & -28.7 & --- & --- & -- & -30.9 & --- & -30.3 & --- \\
\hline-28.0 & --- & -28.7 & -- & -28.6 & --- & -- & -27.2 & -30.9 & --- & -30.4 & --- \\
\hline-27.8 & --- & -28.6 & --- & -28.7 & --- & -- & --- & -30.6 & --- & -30.3 & --- \\
\hline-28.1 & --- & -28.7 & -- & -28.6 & --- & --- & -- & -30.7 & -- & -30.2 & -- \\
\hline-28.0 & --- & -28.9 & --- & -28.7 & -- & --- & -- & -30.6 & --- & -30.3 & -30.1 \\
\hline-28.3 & -- & -28.7 & --- & -28.7 & -- & -- & -- & -30.8 & -- & -30.2 & -29.8 \\
\hline-28.5 & --- & -28.9 & --- & -28.6 & --- & --- & --- & -30.9 & -- & -30.2 & -- \\
\hline-28.2 & --- & -29.0 & --- & -28.5 & --- & -- & $\cdots$ & -30.2 & --- & -30.2 & --- \\
\hline-28.1 & --- & -29.3 & --- & -28.6 & $-\cdots$ & --- & -- & -30.5 & --- & -30.0 & -+- \\
\hline-28.5 & --- & -29.0 & --- & -28.5 & -- & -- & -- & -30.6 & --- & -29.5 & -- \\
\hline-28.4 & --- & -28.8 & --- & -28.6 & --- & -- & -- & -30.5 & --- & -30.0 & --- \\
\hline-28.5 & --- & -28.9 & --- & -28.6 & --- & --- & -- & --- & --- & -29.8 & --- \\
\hline-28.5 & -- & -28.7 & -- & -28.4 & --- & --- & --- & -- & --- & -29.9 & -30.9 \\
\hline-28.4 & -- & --- & --- & -28.7 & --- & --- & -30.9 & -- & -- & -29.8 & -- \\
\hline
\end{tabular}


Table 4.--Scour-hole bed elevations for southeast corner of bent 4, Alsea River at Waldport--Continued

DAILY SCOUR-HOLE ELEVATIONS, FEET, WATER YEAR OCTOBER 1989 TO SEPTEMBER 1990

\begin{tabular}{|c|c|c|c|c|c|c|c|c|}
\hline \multirow[b]{2}{*}{ DAY } & \multicolumn{2}{|c|}{ APRIL } & \multicolumn{2}{|c|}{ MAY } & \multicolumn{2}{|c|}{ JUNE } & \multicolumn{2}{|c|}{ JULY } \\
\hline & RECORDED & OBSERVED & RECORDED & OBSERVED & RECORDED & OBSERVED & RECORI & BSERVED \\
\hline 1 & -30.1 & -- & -30.1 & --- & -- & --- & --- & --- \\
\hline 2 & -30.2 & --- & -- & -31.0 & $-\infty$ & -- & -- & --- \\
\hline 3 & -30.0 & -- & --- & --- & --- & -- & --- & --- \\
\hline 4 & -30.0 & -- & --- & -- & --- & -- & -- & --- \\
\hline 5 & -29.8 & -- & --- & --- & --- & -- & --- & -- \\
\hline 6 & -30.0 & -- & --- & --- & -- & --- & --- & --- \\
\hline 7 & -30.0 & $\sim-$ & --- & -- & -- & -- & --- & -- \\
\hline 8 & -30.1 & --- & --- & --- & --- & --- & --- & --- \\
\hline 9 & -30.2 & -- & --- & --- & -- & -- & --- & -31.3 \\
\hline 10 & -30.0 & -- & --- & --- & --- & --- & --- & --- \\
\hline 11 & -30.0 & -31.1 & --- & -- & --- & --- & --- & $\ldots$ \\
\hline 12 & -29.9 & -- & --- & --- & --- & -31.4 & --- & --- \\
\hline 13 & -30.2 & --- & --- & --- & --- & --- & --- & --- \\
\hline 14 & -30.1 & -- & -- & $-\infty$ & --- & --- & --- & -- \\
\hline 15 & -30.0 & --- & --- & --- & --- & --- & --- & --- \\
\hline 16 & -29.9 & -- & -- & -- & --- & --- & --- & --- \\
\hline 17 & -30.1 & -- & --- & -- & --- & --- & --- & --- \\
\hline 18 & -29.9 & --- & --- & --- & --- & -- & -- & --- \\
\hline 19 & -30.0 & -- & --- & --- & -- & --- & --- & -- \\
\hline 20 & -30.0 & -29.7 & -- & -- & $-\cdots$ & -- & --- & $\ldots$ \\
\hline 21 & -30.0 & --- & --- & --- & --- & --- & --- & --- \\
\hline 22 & -30.1 & -- & --- & --- & --- & --- & --- & -- \\
\hline 23 & -30.0 & --- & --- & --- & -- & --- & -- & -- \\
\hline 24 & -30.1 & --- & -- & -31.2 & $-\cdots$ & -- & --- & -- \\
\hline 25 & -29.8 & -- & --- & -- & --- & -- & $-\infty$ & $-\infty$ \\
\hline 26 & -30.1 & --- & --- & -31.0 & $-\infty$ & -- & -- & --- \\
\hline 27 & -30.0 & --- & -- & -- & --- & -- & -- & -- \\
\hline 28 & -30.2 & -- & --- & --- & $-\infty$ & --- & -- & --- \\
\hline 29 & -30.1 & --- & --- & --- & $-\infty$ & -- & -- & --- \\
\hline 30 & -30.1 & --- & -- & --- & $-\infty$ & $-\infty$ & --- & -- \\
\hline 31 & -- & -- & -- & $\ldots$ & $\ldots$ & --- & -- & --- \\
\hline
\end{tabular}


LOCATION.--Lat $44^{\circ} 25^{\prime} 58^{\prime \prime}$, long $124^{\circ} 04^{\prime} 14^{\prime \prime}$, in SE $1 / 4$ Sw $1 / 4$ sec.18, T.13 S., R.11 W., Lincoln County, Hydrologic unit 17100205 , on a wood pile located 10 feet southwest of bent 4 of new Highway 101 Bridge, 0.5 miles north of Waldport.

PERIOD OF RECORD.--April 1989 to July 1990 (scour-hole elevation).

GAGE.--Bed elevation recorder. Sonic depth sounder in conjunction with a data logger. Datum of gage is 0.00 feet above National Geodetic Vertical Datum of 1929 (levels by oregon Department of Transportation). REMARKS.--Bed elevations at this site indicate local scour from flood tide flows at the southwest corner of bent 4 cofferdam of the new Highway 101 Bridge.

EXTREMES FOR PERIOD OF RECORD.--Maximum bed elevation April 13, 1989 to March 23, 1990, -13.6 feet April 17, 1989; minimum gage height -27.0 feet April 24, 1990.

ELEVATION, IN FEET, WATER YEAR OCTOBER 1988 TO SEPTEMBER 1989

APRIL MAY

JUNE JULY AUGUST

SEPTEMBER

DAY RECORDED OBSERVED RECORDED OBSERVED RECORDED OBSERVED RECORDED OBSERVED RECORDED OBSERVED RECORDED OBSERVED

\begin{tabular}{|c|c|c|c|c|c|c|c|c|c|c|c|}
\hline 1 & --- & -- & -15.5 & $-\infty$ & -16.3 & -- & -16.8 & $-\infty$ & -18.6 & -- & -19.1 \\
\hline 2 & $\ldots$ & $-\infty$ & -16.0 & -- & -16.3 & $\ldots$ & -17 & $-\infty$ & -18.8 & $-\infty$ & -19.3 \\
\hline 3 & -- & $\ldots$ & -15.8 & -14.8 & $-16 \cdot 3$ & $\ldots$ & -17 & $-\infty$ & -18.8 & $-\infty$ & -19.5 \\
\hline 4 & $-\infty$ & $-\infty$ & -14.9 & -- & -16.3 & $\ldots$ & -17.1 & -- & -18.9 & $-\infty$ & -19.4 \\
\hline 5 & $\ldots$ & $-\infty$ & -14.7 & $-\cdots$ & -16.4 & 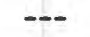 & -17.2 & $\ldots$ & -18.9 & $-\infty$ & -19.3 \\
\hline 6 & $-\cdots$ & -- & -14.4 & -- & -16.4 & -- & $-17 \cdot 3$ & -- & -18.8 & $-\cdots$ & -19.6 \\
\hline 7 & $-\infty$ & $-\infty$ & $-14 \cdot 4$ & -- & -- & -- & -17.5 & $-\infty$ & -18.8 & -- & -19.1 \\
\hline 8 & $-\infty$ & $-\infty$ & -14.4 & $-\cdots$ & -16.6 & -- & -17.2 & $-\infty$ & -18.8 & $-\infty$ & -19.5 \\
\hline 9 & $-\cdots$ & $-\cdots$ & -14.5 & $-\infty$ & -16.6 & -- & -17.3 & $-\infty$ & -18.9 & -- & -19.1 \\
\hline 10 & $\ldots$ & $\cdots$ & -14.5 & $\cdots$ & -16.8 & $\cdots$ & -17.1 & --- & -18.8 & -- & $-19 \cdot 3$ \\
\hline 11 & -- & -- & -14.6 & -- & -16.7 & -- & -16.9 & -- & -18.9 & -- & -19 \\
\hline 12 & $\sim$ & $\ldots$ & -14.7 & $-\infty$ & -16.7 & $\ldots$ & $-17 \cdot 3$ & $-\infty$ & -18.7 & -- & -19.3 \\
\hline 13 & -13.7 & -14.8 & -14.7 & $-\infty$ & -16.7 & -16.9 & -17.2 & $\ldots$ & -18.7 & -- & -19.2 \\
\hline 14 & -13.9 & $-\infty$ & $-15,0$ & -- & -16.6 & -- & -17.1 & $-\sim$ & -17.8 & -- & -19.1 \\
\hline 15 & $-13 \cdot 7$ & $\cdots$ & -14.9 & $\cdots$ & -16.6 & -- & -17.5 & $-\infty$ & -17.7 & -- & -19 \\
\hline 16 & -13.7 & -- & -14.9 & -- & -16.7 & -- & -17.3 & $-\infty$ & -17.7 & -- & -19 \\
\hline 17 & -13.6 & -- & -14.8 & $-\infty$ & -16.7 & $\ldots$ & -17.1 & $-\infty$ & -17.6 & -- & -19 \\
\hline 18 & -13.7 & $-\infty$ & -14.9 & $-\infty$ & -16.6 & -- & -17 & $-\infty$ & -17.7 & -- & -19 \\
\hline 19 & -14.2 & $-\infty$ & -14.9 & -- & -16.6 & & -17.1 & -- & -18.6 & -- & -19.1 \\
\hline 20 & -15.4 & $--\infty$ & -14.9 & -- & -16.6 & & $-17 \cdot 5$ & -- & -19 & -- & -19.3 \\
\hline 21 & -16.0 & -15.8 & -15.1 & -- & -16.7 & & -17.8 & -- & -19.2 & -- & -19.4 \\
\hline 22 & -16.2 & $\therefore$ & -15.0 & -- & -16.6 & $-\infty$ & -17.9 & $-\infty$ & -19.2 & $\ldots$ & -19.5 \\
\hline 23 & -16.0 & -- & -15.2 & -- & -16.6 & $\cdots$ & -17.9 & $\cdots$ & -19.3 & -- & -19.1 \\
\hline 24 & -15.8 & $-\infty$ & -15.1 & -15.3 & -16.8 & $-\infty$ & $-\infty$ & -- & -19.2 & --- & -19.2 \\
\hline 25 & -15.0 & $\cdots$ & -15.0 & $-\cdots$ & -16.8 & & $-\infty$ & $-\infty$ & -19.7 & -- & -18.8 \\
\hline 26 & -14.6 & $-\infty$ & $-15 \cdot 3$ & $--\infty$ & -16.9 & -- & $-\infty$ & $\therefore$ & -19.7 & $-\infty$ & -18.9 \\
\hline 27 & -14.7 & -- & -15.5 & -- & -16.9 & -- & -- & -- & -19.6 & -- & -18.5 \\
\hline 28 & -14.4 & -- & -15.8 & $-\cdots$ & -16.9 & $-\cdots$ & -17.9 & -17.9 & -19.7 & $-\infty$ & -19.2 \\
\hline 29 & -14.5 & -- & -15.9 & $\cdots$ & -16.9 & $\cdots$ & -18.6 & -- & -19.7 & $-\cdots$ & $-19 \cdot 3$ \\
\hline 30 & -15.0 & -- & -15.9 & -- & -16.8 & $-\infty$ & -18.7 & $--\infty$ & -19.3 & -- & -19.1 \\
\hline 31 & -- & $\ldots$ & -16 & -- & -- & $-\ldots$ & -18.7 & - & -19 & -19.7 & - \\
\hline
\end{tabular}


Table 5.--Daily scour-hole bed elevations for SW corner of bent 4 Alsea River at Waldport--Continued

ELEVATION, IN FEET, WATER YEAR OCTOBER 1989 TO SEPTEMBER 1990

\begin{tabular}{|c|c|c|c|c|c|c|c|c|c|c|c|c|}
\hline \multirow[b]{2}{*}{ DAY } & \multicolumn{2}{|c|}{ OCTOBER } & \multicolumn{2}{|c|}{ NOVEMBER } & \multicolumn{2}{|c|}{ DECEMBER } & \multicolumn{2}{|c|}{ JANUARY } & \multicolumn{2}{|c|}{ FEBRUARY } & \multicolumn{2}{|l|}{ MARCH } \\
\hline & RECORDED & OBSERVED & RECORDED & OBSERVED & RECORDED & OBSERVED & RECORDED & OBSERVED & RECORDED & OBSERVED & RECORDED & OBSERVED \\
\hline 1 & -19.0 & --- & -19.5 & -- & -- & -- & -20.6 & -- & -20.7 & --- & -20.7 & -19.7 \\
\hline 2 & -18.6 & --- & -19.5 & --- & --- & -- & -20.7 & -- & -22.1 & -- & -21.0 & --- \\
\hline 3 & -18.7 & --- & -19.6 & $--\infty$ & -- & -- & -20.6 & --- & -21.9 & -- & -21.2 & --- \\
\hline 4 & -19.0 & --- & -19.5 & -- & --- & -- & -20.6 & -- & -21.9 & --- & -21.9 & -- \\
\hline 5 & -19.0 & $-\infty$ & -19.5 & --- & $\ldots$ & $-\cdots$ & -20.5 & --- & -21.8 & --- & -21.2 & -- \\
\hline 6 & -18.6 & --- & -19.4 & --- & --- & -- & -20.6 & $-\ldots$ & $-21 \cdot 7$ & -- & -21.1 & ---6 \\
\hline 7 & -18.8 & --- & -19.4 & -- & --- & -- & -20.6 & --- & -21.8 & --- & -21.1 & --- \\
\hline 8 & -18.8 & -- & -19.4 & --- & $-\cdots$ & -- & -20.5 & --- & -21.8 & -- & -21.2 & -- \\
\hline 9 & -18.5 & --- & -19.4 & --- & --- & -- & -- & -- & -21.7 & --- & -20.9 & -- \\
\hline 10 & -18.7 & -- & -19.3 & -- & -- & $\cdots$ & -- & -- & -- & -- & -21.1 & $-\infty$ \\
\hline 11 & -19.0 & -19.1 & -19.2 & --- & --- & -- & --- & --- & --- & --- & -21.0 & --- \\
\hline 12 & -19.3 & --- & -19.4 & --- & -- & -- & --- & --- & --- & --- & -21.0 & --- \\
\hline 13 & -19.0 & --- & -19.5 & --- & -20.4 & -20.4 & -- & --- & --- & -- & -21.0 & -19.9 \\
\hline 14 & -19.1 & --- & -19.6 & -- & -19.8 & -- & --- & --- & -20.3 & --- & -21.1 & --- \\
\hline 15 & -19.2 & --- & --- & --- & -19.8 & --- & -- & --- & -20.1 & --- & $-21 \cdot 2$ & --- \\
\hline 16 & -19.2 & --- & -- & --- & -19.8 & -- & -- & -- & -20.0 & -- & -21.2 & --- \\
\hline 17 & -19.3 & -- & -19.8 & -- & -19.6 & -- & -20.2 & --- & -19.7 & --- & -21.1 & --- \\
\hline 18 & -19.3 & -- & -19.9 & -- & -19.8 & -- & -20.1 & --- & -20.2 & --- & -21.3 & --- \\
\hline 19 & -19.2 & --- & -- & --- & -19.9 & -- & -20.2 & -19.9 & -20.1 & -- & -21.3 & -- \\
\hline 20 & -19.2 & $-\cdots$ & -- & --- & -19.8 & -- & -20.2 & -- & -20.1 & --- & -21.3 & --- \\
\hline 21 & -19.4 & --- & --- & -- & -19.9 & -- & -20.1 & -- & -19.6 & -- & -21.4 & -- \\
\hline 22 & -19.1 & --- & -- & -- & -19.8 & -- & -20.4 & --- & -20.1 & --- & -21.2 & --- \\
\hline 23 & -19.2 & --- & --- & --- & -19.9 & -- & -20.5 & -- & -20.3 & --- & -21.3 & -19.4 \\
\hline 24 & -19.4 & --- & --- & --- & -19.7 & -- & -20.2 & -- & -20.3 & -- & -21.6 & -- \\
\hline 25 & -19.4 & -- & -- & -- & -19.9 & -- & -20.6 & -- & -20.4 & --- & -22.1 & -- \\
\hline 26 & -19.4 & --- & --- & -- & -19.7 & -- & -20.5 & -- & -20.4 & $-\cdots$ & -24.0 & -- \\
\hline 27 & -19.5 & --- & --- & -- & -19.9 & -- & -20.6 & --- & -20.6 & --- & -24.0 & --- \\
\hline 28 & -19.6 & --- & --- & $-\infty$ & -19.8 & $-\infty$ & -20.5 & --- & -20.6 & --- & -24.7 & --- \\
\hline 29 & -19.4 & --- & -- & --- & -19.9 & -- & -20.6 & --- & -- & --- & -24.9 & -- \\
\hline 30 & -19.4 & --- & -- & -- & -19.8 & -- & -20.6 & -- & --- & -- & -25.2 & -24.9 \\
\hline 31 & -19.4 & -- & --- & $-\infty$ & -19.9 & -- & -20.5 & -22.6 & -- & --- & -24.9 & -- \\
\hline
\end{tabular}


Table 5.--Daily-scour-hole bed elevations for southwest corner of bent 4 , Alsea River at Waldport--Continued

ELEVATION, IN FEET, WATER YEAR OCTOBER 1989 TO SEPTEMBER 1990 \begin{tabular}{ccccc}
\multicolumn{2}{c}{ APRIL } & \multicolumn{2}{c}{ MUY JUNE } \\
RECORDED OBSERVED RECORDED & OBSERVED RECORDED OBSERVED RECORDED OBSERVED
\end{tabular} \begin{tabular}{ccccc}
\multicolumn{2}{c}{ APRIL } & \multicolumn{2}{c}{ MUY } & \multicolumn{2}{c}{ JULY } \\
RECORDED OBSERVED RECORDED & OBSERVED RECORDED OBSERVED RECORDED OBSERVED
\end{tabular} \begin{tabular}{ccccc}
\multicolumn{2}{c}{ APRIL } & \multicolumn{2}{c}{ MUY JULY } \\
RECORDED & \multicolumn{1}{c}{ JUSERVED RECORDED OBSERVED RECORDED OBSERVED RECORDED OBSERVED }
\end{tabular} \begin{tabular}{ccccc}
\multicolumn{2}{c}{ APRIL } & \multicolumn{2}{c}{ MUY JUNE } \\
RECORDED OBSERVED RECORDED & OBSERVED RECORDED OBSERVED RECORDED OBSERVED
\end{tabular}

$-26.4$

25

$-25.1$

$-26.1$

$-22.1$

$-23.2$

$-22 \cdot 8$

-- $\quad-21.8 \quad-\cdots$

$-26.0$

$-21.8$

$-22.2$

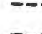

$-21.6 \quad-22$.

$-$

$-26.3 \quad \ldots$

$-21.8$

$-22.4$

...

$-21.9$

$\begin{array}{ll}-22.0 & -- \\ -21.9 & --\end{array}$

$-26.1 \quad \ldots$

$-22.0$

$-26.1$

$-21.9$

-..

$-22$

$-21.7 \quad-\cdots$

$-26.1$

$-21.6$

--.

$-22.7 \quad--$

$-22.5$

$-$

-22 .

$-22.0$

-n

$-\cdots$

$-21.6 \quad--$

$-26.1 \quad-$.

$-22.5$

$-$

$-22.2$

$-22.4$

$-26.1$

$-26.3$

$-21.7$

--

-22.4
-23.0

$--$

$-21.6$

$-21.8$

26.5

$-21.5$

$-22.4$

$-26.4$

$-$

$-22.2$

$-26.3$

$-22.3$

$-26.5$

$--$

$-22.9$

$-26.7$

$-\infty$

-22.4
-22.4

$-26.3$

$-25.8$

-22.8
-22.8

$\ldots \quad-23.0$

$--$

-22 .

-23.6
-22.9

$-23.4$

$-23.7$

$-22.7$

-22 .

$-22.4$

$-22.2$

$-26.5$

$-21.9$

$-26.3$

$-22.7$

$-22.7$

$-22.4$

$-23.3$

$-22.1$

$-22.2$

$-27.0$

-

$-26.1$

$--$

$-22.7$

$-25.7$

--

$-23.1$

$-26.1$

$-$

$-23.5$

$-22.6$

-

$-22.7$

$-22.6$ 
LOCATION.--Lat $40^{\circ} 25^{\prime} 58^{\prime \prime}$, long $124^{\circ} 04^{\prime} 14^{\prime \prime}$, in SE $1 / 4$ SW $1 / 4$ sec.18, T.13 S., R.11 w., Lincoln County, Hydrologic Unit 17100205, on a wooden pile located 40 feet east of bent 4 of new Highway 101 Bridge, 0.5 miles north of waldport.

PERIOD OF RECORD.--April 1989 to March 1990 (bed elevation)

GAGE.--Bed elevation recorder. Sonic depth sounder in conjunction with a data logger. Datum of gage is 0.00 feet above National Geodetic Vertical Datum of 1929 (levels by oregon Department of Transportation).

REMARKS.--Bed elevations at this site reflect contraction scour. These data are used to help differentiate between general scour, constriction and local scour at bent 4 of the new Highway 101 Bridge.

EXTREMES FOR PERIOD OF RECORD.--Maximum daily bed elevation April 13, 1989 to Marrch 23, 1990, -10.7 feet

January 8, 1990; minimum daily bed elevation -15.1 feet July 26, August 7,8,11,12, 1989 .

DAILY ELEVATION, IN FEET, WATER YEAR OCTOBER 1988 TO SEPTEMBER 1989

APRIL MAY JUNE JULY AUGUST

DAY RECORDED OBSERVED RECORDED OBSERVED RECORDED OBSERVED RECORDED OBSERVED RECORDED OBSERVED RECORDED OBSERVED

\begin{tabular}{|c|c|c|c|c|c|c|c|c|c|c|c|c|}
\hline 1 & -.- & --- & -12.9 & --- & -13.0 & -- & -14.9 &.- & -14.9 & -- & -13.2 & -- \\
\hline 2 & --- & -.- & -12.9 & -- & -13.5 & --- & -14.8 & -- & -14.8 & -- & -13.1 & --- \\
\hline 3 & --- & -- & -12.9 & -12.4 & -13.8 & -- & -14.7 & -- & -14.9 & -- & -13.1 & --- \\
\hline 4 & -- & --- & -12.9 & --- & -13.6 & -- & -14.7 & -- & -14.8 & -- & -13.1 & --- \\
\hline 5 & -- & -- & -13.0 & -- & --- & -- & -14.8 & $\cdots$ & -14.9 & - & -13.1 & -.- \\
\hline 6 & -- & -- & -13.2 & --- & -14.3 & -- & -14.9 & -- & -15.0 & $-\cdots$ & -13.2 & --- \\
\hline 7 & $\cdots$ &.- & -13.6 & -- & -14.4 & -- & -14.8 & -- & -15.0 & -- & -13.2 & -- \\
\hline 8 & -- & -- & -13.6 &.-- & -14.5 & -- & -14.9 & -- & -15.1 & -- & -13.2 & $-\cdots$ \\
\hline 9 & -- & -- & -13.7 & --- & -14.6 & --- & -14.8 & -- & -15.1 & -- & -13.2 & -- \\
\hline 10 & --- & --- & -13.6 & $\cdots$ & -14.7 & -- & -14.7 & -- & -15.0 & -- & -13.1 & --- \\
\hline 11 & -- & -- & -13.6 & -- & -14.8 & -- & -14.7 & --- & -15.1 & -- & -13.1 & -- \\
\hline 12 & --- & -- & -13.6 & -- & -14.8 & --- & -14.7 & -- & -15.1 & -- & -12.9 & -- \\
\hline 13 & -13.7 & -14.0 & -13.6 & -- & -14.8 & -- & -14.6 & -- & -14.9 & -- & -13.0 & -- \\
\hline 14 & -14.0 & -- & -13.6 & -- & -14.7 & -14.8 & -14.6 & -- & -14.8 & -- & -12.8 & --- \\
\hline 15 & -14.0 & -- & -13.6 & --- & -14.7 & -- & -14.5 & $-\infty$ & -14.7 & $-\infty$ & -12.3 & $-\infty$ \\
\hline 16 & -14.1 & -- & -13.5 & - & -14.5 & -- & -14.7 & -- & -14.7 & -.- & -12.2 & -- \\
\hline 17 & -14.1 & -- & -13.4 & -- & -14.4 & -- & -14.6 & -- & -14.7 & -- & -12.4 & -- \\
\hline 18 & -14.0 & $\cdots$ & -13.3 &.- & -14.3 & -- & -14.5 & -- & -14.6 & -- & -12.2 & -- \\
\hline 19 & -14.0 & -- & -13.2 & -- & -14.2 & -- & -14.5 & -- & -14.3 & -- & -12.8 & -- \\
\hline 20 & -13.9 & -- & -13.2 & --- & -14.4 & -- & -14.5 & -- & -14.2 & -- & -- & --- \\
\hline 21 & -13.7 & -- & -13.2 & -- & -14.4 & -- & -14.7 & -.- & -14.0 & --- & -13.0 & -- \\
\hline 22 & -13.5 & $\cdots$ & -13.2 & -- & -14.4 & -- & -14.7 & -- & -13.9 & -- & -12.3 & --- \\
\hline 23 & -- & $\cdots$ & -13.2 & -- & -14.6 & -- & -14.7 & $\cdots$ & -13.3 & 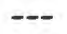 & -12.2 & -- \\
\hline 24 & -13.1 & -- & -13.2 & $\cdots$ & -14.6 & -.. & -14.7 & -- & -13.2 & -- & -12.4 & -- \\
\hline 25 & -13.8 & $\cdots$ & -13.2 & -- & -14.8 & -- & -14.9 & $-\infty$ & -13.1 & -- & -12.6 & $\cdots$ \\
\hline 26 & -14.4 & -- & -13.2 & -- & -14.8 & --- & -15.1 & $-\cdots$ & -13.1 & -- & -- & -- \\
\hline 27 & -12.9 & -- & -13.3 & -- & -15.0 & -- & -15.0 & $\ldots$ & -13.2 & -- & -- &.-- \\
\hline 28 & -12.9 & -- & -13.4 & --- & -14.9 & -- & -15.0 & -- & -13.2 & -- & -12.7 & -- \\
\hline 29 & -12.9 & -- & -13.4 & --- & -14.9 & --- & -14.9 & -- & -13.2 & $\cdots$ & -- & -- \\
\hline 30 & -13.0 & $\cdots$ & -13.5 & -- & -14.8 & -- & -14.9 & -- & -13.2 & -- & $\cdots$ & -- \\
\hline 31 & --- & -- & -13.4 & $\cdots$ & -- & -- & -14.9 & -. & -13.1 & -- & -- & -- \\
\hline
\end{tabular}


Table 6.--Bed elevations for 40 ft east of bent 4, Alsea River at Waldport--Continued DAILY ELEVATION, IN FEET, WATER YEAR OCTOBER 1989 TO SEPTEMBER 1990

OCTOBER

NOVEMBER

DECEMBER JANUARY

FEBRUARY

MARCH

DAY RECORDED OBSERVED RECORDED OBSERVED RECORDED OBSERVED RECORDED OBSERVED RECORDED OBSERVED RECORDED OBSERVED

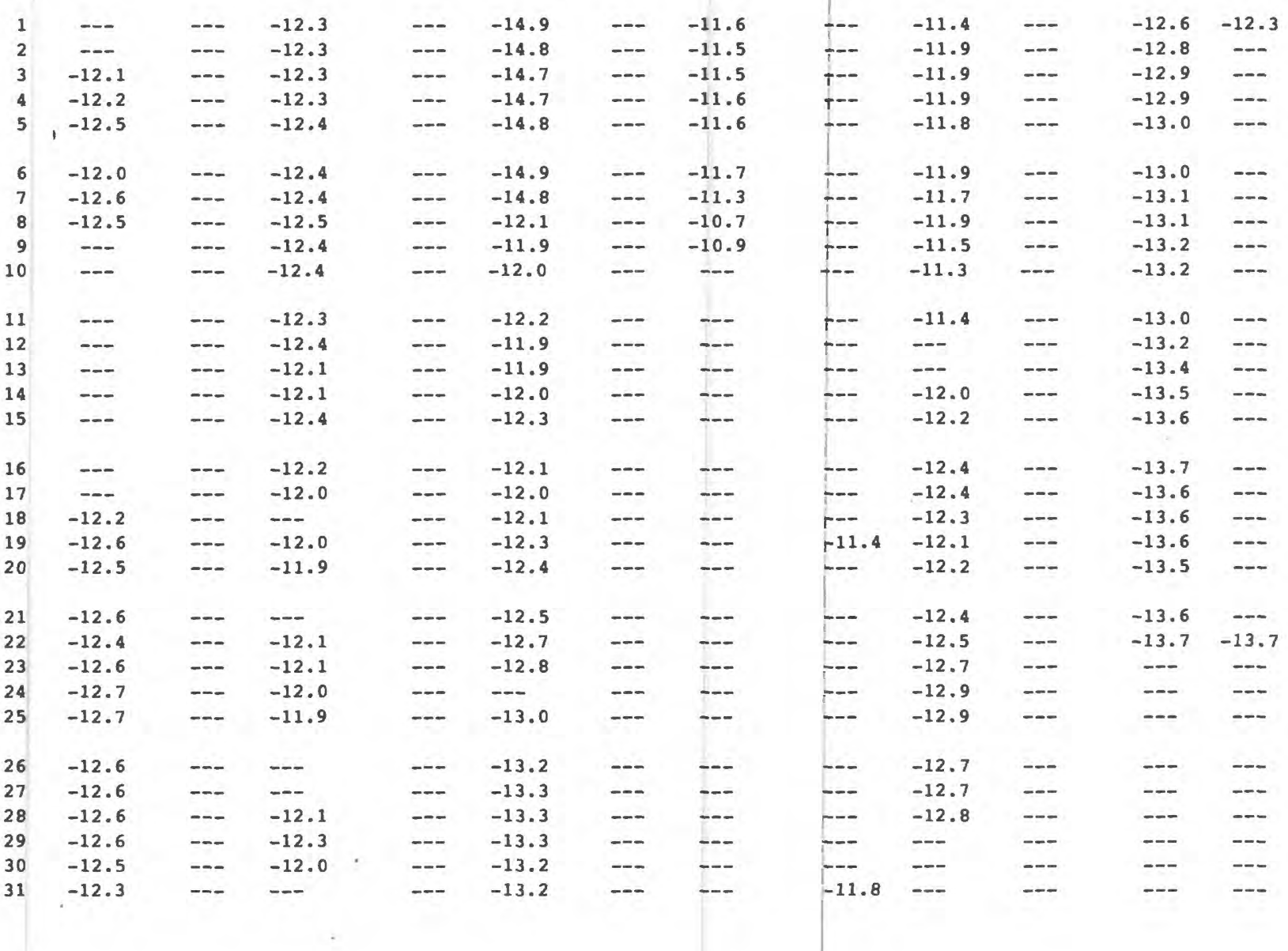


LOCATION.--Lat $44^{\circ} 25^{\prime} 58^{\prime \prime}$, long $124^{\circ} 04^{\prime} 14^{\prime \prime}$, in SE $1 / 4$ SW $1 / 4$ sec.18, T.13 S., R.11 W., Lincoln County, Hydrologic Unit 17100205, on bent 3 of Highway 101 Bridge (under construction), $0.5 \mathrm{mile}$ north of waldport.

PERIOD OF RECORD.--May 3-23, 1989 (scour-hole elevations) discontinued.

GAGE.--Portable depth sounder and data logger. Datum of gage is 0.00 feet above National Geodetic Vertical Datum of 1929 (levels by Oregon Department of Transportation).

REMARKS.--Data were collected at this site to compare with scour-hole data collected at bent 4 , which is located 350 feet south of this location.

EXTREMES FOR PERIOD OF RECORD.--Maximum daily elevation recorded, -14.9 feet May 3, 1989; minimum gage height recorded -15.6 feet May 23, 1989.

ELEVATION, IN FEET, WATER YEAR OCTOBER 1988 TO SEPTEMBER 1989

DAILY MEAN VALUES

MARCH APRIL MAY JUNE JULY

DAY RECORDED OBSERVED RECORDED OBSERVED RECORDED OBSERVED RECORDED OBSERVED RECORDED OBSERVED

\begin{tabular}{|c|c|c|c|c|c|c|c|c|c|c|}
\hline 1 & - - & -- & -- & -- & -- & -- & -- & -- & -- & -- \\
\hline 2 & --- & --- & $-\infty$ & -- & -- & -- & -- & -- & -- & -- \\
\hline 3 & $-\infty$ & --- & -- & --- & -14.9 & -- & -- & -- & -- & -- \\
\hline 4 & -- & -- & -- & -- & -15.3 & -- & -- & -- & -- & -- \\
\hline 5 & -- & --- & -- & --- & -15.3 & -- & -- & -- & -- & -- \\
\hline 6 & -- & -- & -- & --- & -15.3 & -- & --- & $-\infty$ & $\ldots$ & -- \\
\hline 7 & $-\infty$ & -- & -- & -- & -15.4 & $-\infty$ & --- & -- & -- & -- \\
\hline 8 & -- & --- & -- & -- & -15.3 & --- & -- & -- & -- & -- \\
\hline 9 & --- & -- & -- & -- & -15.3 & -- & -- & -- & -- & -- \\
\hline 10 & -- & -- & -- & -- & -15.4 & -- & -- & -- & -- & -- \\
\hline 11 & $-\infty$ & -- & -- & $-\infty$ & -15.4 & -- & -- & -- & -- & -- \\
\hline 12 & -- & -- & -- & -- & -15.4 & $=-$ & -- & -- & -- & --- \\
\hline 13 & -- & $-\infty$ & $-\infty$ & -- & -15.4 & $-\infty$ & $-\infty$ & -- & -- & -- \\
\hline 14 & $-\infty$ & -- & -- & -- & -15.3 & -- & -- & -- & -- & --- \\
\hline 15 & -- & -- & $-\infty$ & -- & -15.3 & $\cdots$ & -- & -- & -- & -- \\
\hline 16 & -- & -- & $-\infty$ & -- & -15.3 & --- & -- & -- & -- & -- \\
\hline 17 & -- & -- & $-\infty$ & -- & -15.4 & -- & -- & --- & --- & -- \\
\hline 18 & $-\infty$ & -- & -- & $=-$ & -15.3 & -- & -- & -- & -- & -- \\
\hline 19 & -- & $-\infty$ & -- & -- & -15.4 & $--\infty$ & -- & $-\infty$ & $=-$ & $-\infty$ \\
\hline 20 & -- & $-\infty$ & --- & $-\infty$ & $-15 \cdot 3$ & -- & -- & -- & -- & -- \\
\hline 21 & $-\infty$ & --- & -- & -- & -15.3 & -- & -- & -- & -- & -- \\
\hline 22 & -- & -- & -- & -- & -15.3 & $-\infty$ & $-\infty$ & -- & -- & -- \\
\hline 23 & $-\infty$ & -- & -- & $=-$ & -15.6 & $-\infty$ & -- & $--\infty$ & -- & -- \\
\hline 24 & $-\infty$ & -- & --- & -- & -- & -- & $\ldots$ & -- & -- & $-\cdots$ \\
\hline 25 & -- & $-\infty$ & -- & -- & -- & -- & -- & -- & -- & -- \\
\hline 26 & --- & -- & -- & -- & -- & -- & -- & $-a-$ & -- & -- \\
\hline 27 & -- & -- & --- & -- & -- & $-\infty$ & -- & -- & -- & -- \\
\hline 28 & -- & -- & -- & -- & -- & -- & $-\infty$ & -- & -- & -- \\
\hline 29 & -- & -- & -- & -- & $-\infty$ & -- & -- & -- & -- & -- \\
\hline 30 & $-\infty$ & -- & -- & -- & -- & -- & -- & -- & -- & -- \\
\hline 31 & -- & -- & --- & $-\infty$ & -- & -- & $-\infty$ & -- & -- & -- \\
\hline
\end{tabular}




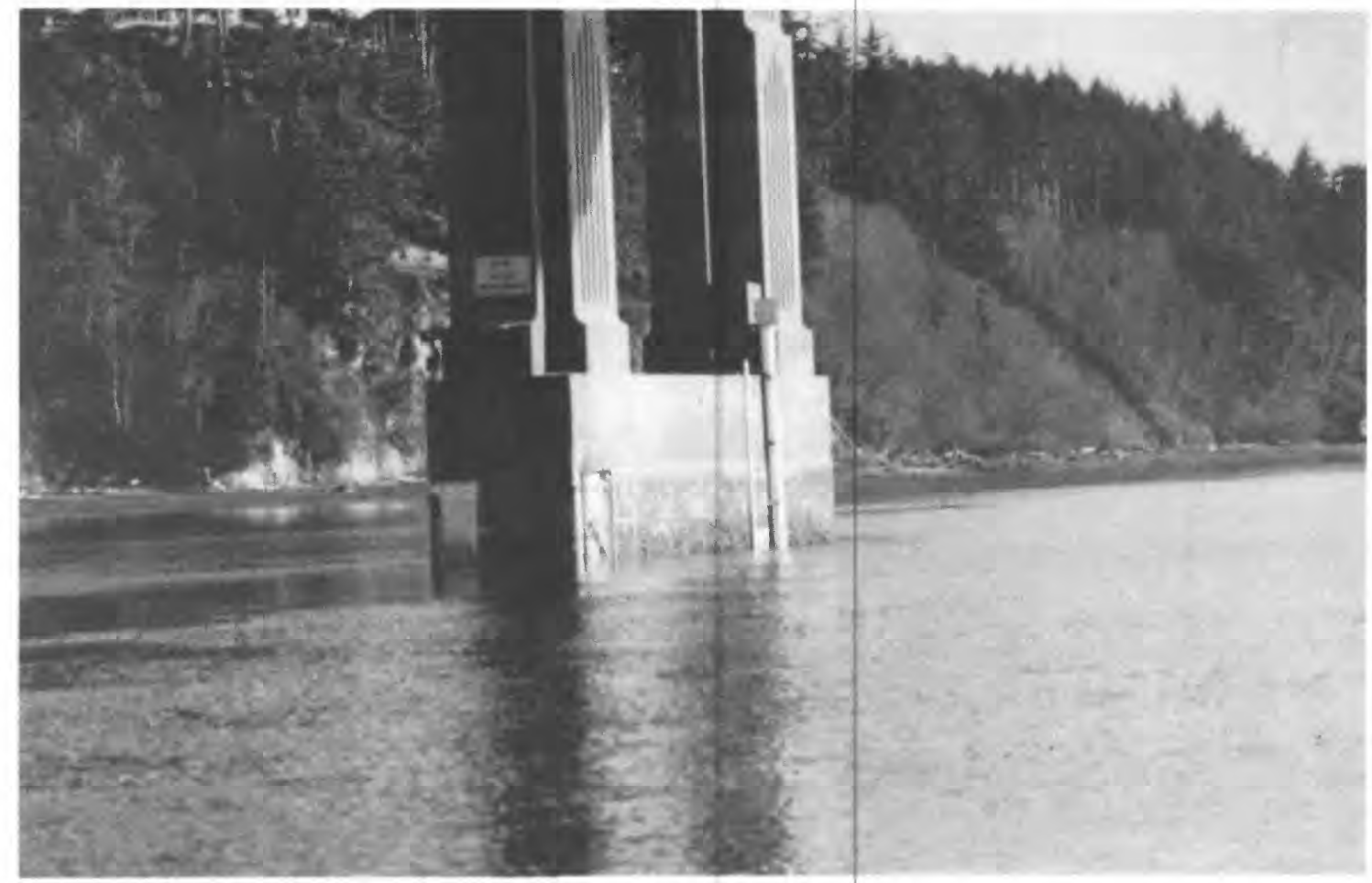

Instrument shelter, stilling well, and velocity probe support at pier 6 at Highway 101 bridge over Alsea Bay. Photo taken before construction of new bridge.

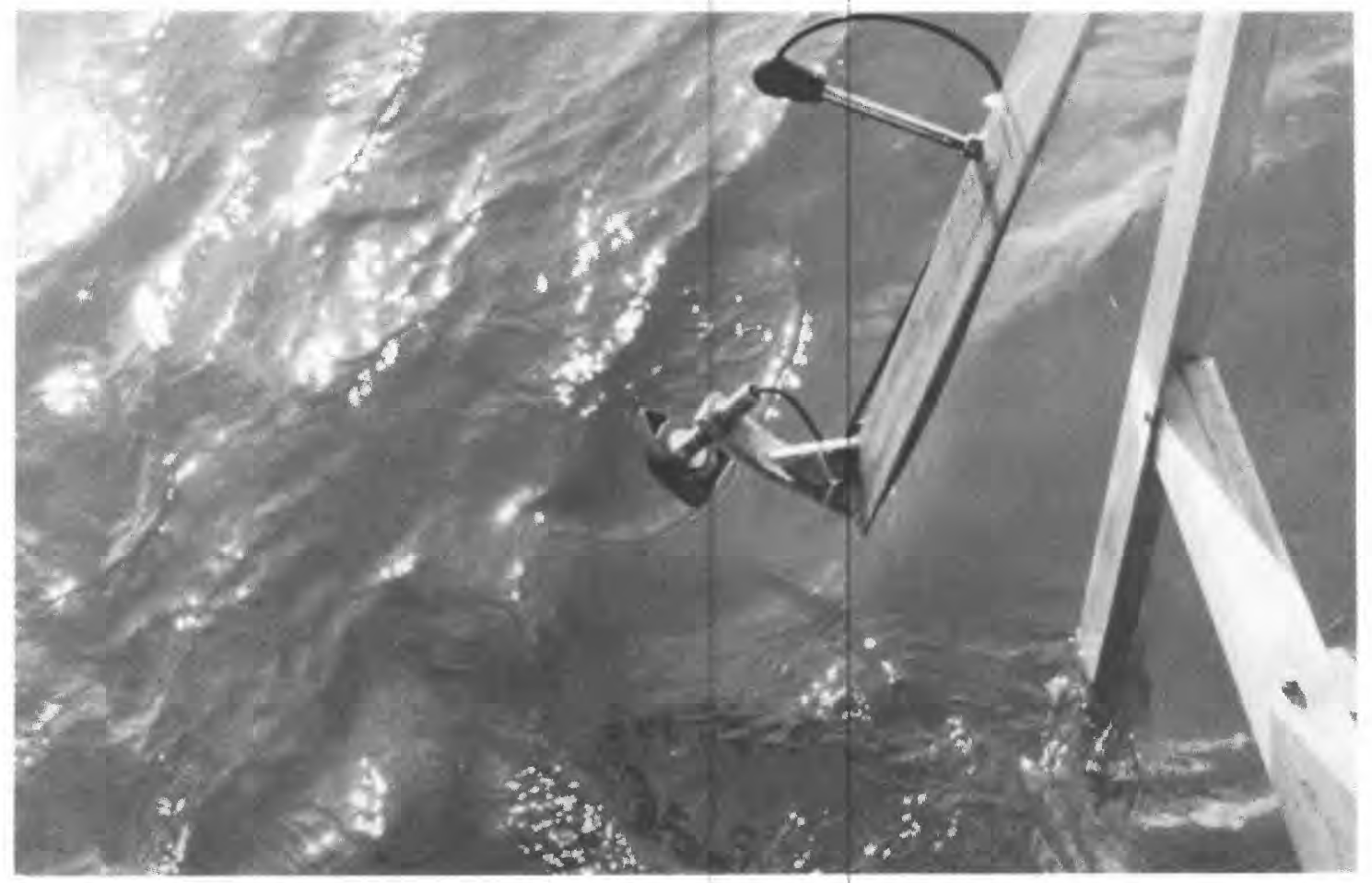

Velocity and depth transducer mounted on aluminum angle for support. 


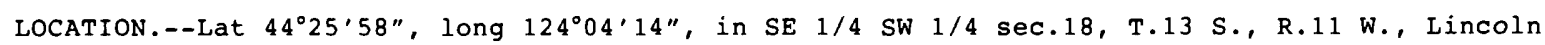
County, Hydrologic Unit 17100205, on pier 6 of Highway 101 Bridge, 0.5 miles north of waldport.

PERIOD OF RECORD.--March 1988 to July 1990 (gage height).

GAGE.--Water-stage recorder. Datum of gage is 0.00 feet above National Geodetic Vertical Datum of 1929 (levels by Oregon Department of Transportation).

REMARKS.--Site is affected by tides.

EXTREMES FOR PERIOD OF RECORD.--Maximum gage height, 8.18 feet November 22,1988 ; minimum gage height -5.60 feet June 19,1989 .

GAGE HEIGHT, IN FEET, WATER YEAR OCTOBER 1987 TO SEPTEMBER 1988

\begin{tabular}{|c|c|c|c|c|c|c|c|c|c|c|c|c|}
\hline \multirow[b]{2}{*}{ DAY } & \multicolumn{2}{|c|}{ OCTOBER } & \multicolumn{2}{|c|}{ NOVEMBER } & \multicolumn{2}{|c|}{ DECEMBER } & \multicolumn{2}{|c|}{ JANUARY } & \multicolumn{2}{|c|}{ FEBRUARY } & \multicolumn{2}{|c|}{ MARCH } \\
\hline & $\operatorname{MAX}$ & MIN & $\operatorname{MAX}$ & MIN & $\operatorname{MAX}$ & MIN & $\operatorname{MAX}$ & MIN & MAX & MIN & $\operatorname{MAX}$ & MIN \\
\hline 1 & $\ldots$ & $\ldots$ & --- & $\ldots$ & -- & --- & -- & --- & --- & --- & -- & -- \\
\hline 2 & --- & --- & -- & --- & -- & --- & --- & --- & --- & --- & 4.42 & -2.64 \\
\hline 3 & --- & --- & --- & --- & --- & --- & --- & --- &.-- & -- & 4.56 & -2.79 \\
\hline 4 & --- & -- &.- & --- &.- &.-- & --- & --- &.-- & --- & 3.97 & -2.72 \\
\hline 5 & --- & -- & -- & -- &.- & --- & --- & --- & --- & --- & 4.68 & -1.39 \\
\hline 6 & --- & -- & -- & -- & -- & -- & --- & --- & --- & --- & 5.36 & -1.67 \\
\hline 7 & --- & --- & --- & --- & --- & --- & --- & --- & --- & --- & 4.54 & -2.62 \\
\hline 8 & --- & -- & -- & -- & --- & --- & --- & --- & --- & --- & 4.21 & -2.75 \\
\hline 9 & --- & -- & -- & -- & --- & --- & --- & --- & --- & --- & 4.29 & -2.60 \\
\hline 10 & --- & -- & -- & -- & --- & --- & -- & -- & --- & --- & 4.19 & -2.83 \\
\hline 11 & --- & -- & -- & -- & --- & --- & --- & --- & --- & --- & 3.44 & -3.10 \\
\hline 12 & --- & -- & --- & --- & --- & -- & --- & --- & --- & -- & 3.85 & -3.30 \\
\hline 13 & --- & --- & --- & --- & --- & --- & --- & --- & --- & --- & 3.65 & -3.76 \\
\hline 14 & -- & -- & -- & --- & --- & -- & --- & --- & --- & -- & 3.86 & -4.47 \\
\hline 15 & --- & --- & --- & --- & --- & --- & $\cdots$ & $\cdots$ & -- & -- & -- & --- \\
\hline 16 & --- & --- & --- & --- & --- & --- & --- & --- & --- & --- & --. & --- \\
\hline 17 & --- & -- & -- & -- & --- & --- & --- & --- & --- & --- & 4.47 & -4.33 \\
\hline 18 & --- & -- & -- & -- & --- & --- & --- & --- & --- & --- & 4.53 & -3.76 \\
\hline 19 & -.. & -. & -- & --- & --. & --- & --- & --- & --. & --- & 4.94 & -3.79 \\
\hline 20 & $\cdots$ & $\cdots$ & $\cdots$ & -- & --- & -- & --- & --- & --- & -- & 5.63 & -3.66 \\
\hline 21 & -- & -- & -- & -- & --- & --- & --- & --- & --- & -- & 5.40 & -3.51 \\
\hline 22 & --- & --- & --- & --- & --- & --- & --- & --- & --- & -- & 5.63 & -2.92 \\
\hline 23 & -- & $\cdots$ & -- & -- & --- & -- & --- & --- & --- & --- & 5.57 & -1.82 \\
\hline 24 & -- & -- & -- & -- & --- & --- & --- & --- & --- & --- & 4.68 & -2.39 \\
\hline 25 & -- & --- & -- & --- & -- & --- & -- & --- & -- & -- & 3.60 & -1.83 \\
\hline 26 & -- & -- & -- & -- & --- & --- & --- & --- & --- & --- & 3.45 & -1.98 \\
\hline 27 & --- & --- & --- & -- & --- & -- & --- & -- & -- & --- & 3.02 & -2.46 \\
\hline 28 & -.- & -- & -- & --- & -- & --- & --- & --- & --- & --- & 2.58 & -3.10 \\
\hline 29 & -- & -- & -- & -- & -- & --- & --- & --- & --- & --- & 2.99 & -2.80 \\
\hline 30 & --- & --- & --- & -- & -- & -- & --- & --- & --- & --- & 2.95 & -3.12 \\
\hline 31 & --- & -- & --- & -- & -- & -- & -- & --- & --- & -- & 3.27 & -3.05 \\
\hline MON & CH-D- & --- & --- & -- & -.. & --- & --- & --- & --- & --. & -.- & --- \\
\hline
\end{tabular}


Table 8.--Maximum and minimum daily water levels for Alsea River at Waldport--Continued

GAGE HEIGHT, IN FEET, WATER YEAR OCTOBER 1987 TO SEPTEMBER 1988

\begin{tabular}{|c|c|c|c|c|c|c|c|c|c|c|c|c|}
\hline \multirow[b]{2}{*}{ DAY } & \multicolumn{2}{|c|}{ APRIL } & \multicolumn{2}{|c|}{ MAY } & \multicolumn{2}{|c|}{ JUNE } & \multicolumn{2}{|c|}{ JULY } & \multicolumn{2}{|c|}{ AUGUST } & \multicolumn{2}{|c|}{ SEPTEMBER } \\
\hline & $\operatorname{MAX}$ & MIN & $\operatorname{MAX}$ & MIN & MAX & MIN & $\operatorname{MAX}$ & MIN & $\operatorname{MAX}$ & MIN & MAX & MIN \\
\hline 1 & 4.02 & -2.50 & 4.85 & -3.62 & 5.57 & -4.19 & 4.83 & -5.00 & 4.25 & -4.35 & 4.18 & -3.25 \\
\hline 2 & 5.34 & -2.47 & 5.35 & -3.96 & 5.69 & -4.33 & 4.95 & -4.84 & 4.35 & -3.54 & 3.53 & -3.22 \\
\hline 3 & 5.57 & -1.80 & 5.23 & -3.68 & 6.55 & -3.57 & 4.50 & -4.81 & 4.40 & -2.83 & 3.17 & -3.36 \\
\hline 4 & 5.45 & -2.97 & 5.20 & -4.12 & 5.15 & -4.27 & 3.64 & -4.31 & 4.47 & -2.63 & 3.05 & -3.47 \\
\hline 5 & 4.75 & -3.34 & 4.75 & -4.26 & 4.07 & -4.08 & 3.83 & -3.60 & 4.14 & -2.86 & 3.02 & -3.65 \\
\hline 6 & 5.15 & -2.93 & 4.35 & -4.16 & 3.60 & -3.46 & 3.90 & -2.88 & 3.88 & -3.27 & 3.05 & 3.82 \\
\hline 7 & 4.53. & -3.49 & 4.14 & -3.64 & 3.71 & -3.16 & 4.08 & -2.88 & 3.73 & -3.53 & 3.32 & -3.74 \\
\hline 8 & 3.74 & -3.67 & 3.57 & -3.67 & 4.03 & -2.67 & 4.00 & -3.28 & 3.67 & -3.94 & 3.38 & -3.59 \\
\hline 9 & 3.13 & -3.82 & 3.26 & -3.16 & 4.57 & -2.52 & 4.05 & -4.01 & 3.89 & -4.22 & 3.33 & -3.62 \\
\hline 10 & 3.25 & -3.21 & 3.60 & -3.05 & 4.40 & -3.28 & 4.31 & -4.39 & 4.07 & -4.15 & 3.09 & -3.52 \\
\hline 11 & 2.93 & -3.59 & 4.48 & -2.49 & 4.66 & -4.21 & 4.45 & -4.41 & 4.06 & -4.07 & 3.34 & -3.12 \\
\hline 12 & 3.53 & -3.56 & 5.18 & -2.27 & 4.53 & -4.62 & 4.70 & -4.45 & 3.90 & -4.07 & 4.00 & -2.60 \\
\hline 13 & 4.76 & -2.68 & 5.50 & -2.75 & 4.50 & -5.00 & 4.56 & -4.39 & 3.96 & -4.00 & 3.94 & -2.61 \\
\hline 14 & 5.16 & -2.49 & 5.70 & -3.80 & 4.35 & -4.97 & 4.38 & -4.37 & 3.60 & -3.78 & 3.86 & -2.97 \\
\hline 15 & 5.34 & -3.06 & 5.77 & -4.06 & 4.36 & -5.00 & 4.28 & -4.49 & 3.18 & -3.36 & 3.69 & -3.04 \\
\hline 16 & 5.34 & -3.43 & 5.56 & -4.24 & 4.27 & -4.80 & 3.64 & -4.51 & 3.30 & -2.78 & 3.61 & -3.23 \\
\hline 17 & 6.00 & -4.10 & 5.53 & -4.35 & 4.03 & -4.36 & 3.02 & -4.41 & 3.22 & -2.51 & 3.34 & -3.33 \\
\hline 18 & 5.30 & -4.60 & 5.08 & -4.26 & 3.79 & -3.96 & 2.56 & -3.92 & 2.96 & -2.77 & 3.18 & -2.98 \\
\hline 19 & 5.39 & -4.09 & 4.29 & -4.41 & 3.10 & -3.69 & 3.02 & -3.04 & 3.01 & -2.84 & 3.43 & -2.95 \\
\hline 20 & 5.04 & -3.78 & 3.40 & -4.27 & 2.75 & $-3.2^{7}$ & 3.05 & -2.45 & 3.15 & -2.72 & 3.40 & -3.48 \\
\hline 21 & 4.53 & -3.37 & 3.22 & -3.41 & 2.62 & -2.68 & 2.88 & -2.36 & 3.48 & -2.98 & 3.53 & -3.91 \\
\hline 22 & 3.76 & -3.05 & 2.80 & -3.18 & 3.37 & -2.00 & 3.01 & -2.47 & 3.86 & -3.15 & 3.80 & -3.99 \\
\hline 23 & 3.09 & -2.79 & 2.26 & -2.98 & 3.50 & -1.36 & 3.29 & -2.94 & 4.19 & -3.44 & 4.30 & -3.86 \\
\hline 24 & 2.26 & -2.67 & 2.66 & -2.52 & 3.75 & -2.21 & 3.67 & -3.37 & 4.47 & -3.96 & --- & --- \\
\hline 25 & 2.46 & -2.68 & 3.15 & -2.09 & 4.05 & -2.86 & 4.43 & -3.82 & 4.78 & -4.45 & 5.25 & -3.28 \\
\hline 26 & 3.09 & -2.38 & 3.96 & -1.77 & 3.99 & -3.67 & 4.77 & -4.16 & 4.88 & -4.68 & 5.00 & -3.97 \\
\hline 27 & 3.63 & -1.88 & 4.62 & -1.76 & 4.25 & -4.50 & 4.87 & -4.68 & 4.53 & -4.76 & 5.06 & -4.46 \\
\hline 28 & 4.22 & -1.47 & 4.81 & -2.30 & 4.59 & -4.98 & 4.90 & -5.00 & 4.67 & -4.54 & 5.06 & -4.37 \\
\hline 29 & 4.42 & -2.02 & 5.09 & -3.25 & 4.76 & -5.00 & 4.72 & -5.00 & 4.65 & -3.83 & 5.44 & -3.69 \\
\hline 30 & 4.56 & -2.93 & 5.36 & -3.81 & 4.68 & -5.00 & 4.83 & -5.00 & 5.37 & -3.05 & 4.65 & -3.52 \\
\hline 31 & --- & --- & 5.66 & -4.18 & --- & --- & 4.74 & -5.00 & 4.69 & -3.25 & --- & --- \\
\hline MON & .00 & -4.60 & 5.77 & 4.41 & 6.55 & -5.00 & 4.95 & -5.00 & 5.37 & -4.76 & --- & --- \\
\hline
\end{tabular}


Table 8.--Maximum and minimum daily water levels for the Alsea River at Waldport--Continued

GAGE HEIGHT, IN FEET, WATER YEAR OCTOBER 1988 TO SEPTEMBER 1989

\begin{tabular}{|c|c|c|c|c|c|c|c|c|c|c|c|c|}
\hline \multirow[b]{2}{*}{ DAY } & \multicolumn{2}{|c|}{ APRIL } & \multicolumn{2}{|c|}{ MAY } & \multicolumn{2}{|c|}{ JUNE } & \multicolumn{2}{|c|}{ JULY } & \multicolumn{2}{|c|}{ AUGUST } & \multicolumn{2}{|c|}{ SEPTEMBER } \\
\hline & MAX & MIN & MAX & MIN & MAX & MIN & MAX & MIN & $\operatorname{MAX}$ & MIN & MAX & MIN \\
\hline 1 & 4.79 & -1.30 & 4.07 & -2.86 & 4.98 & -4.14 & 5.48 & -4.25 & 4.35 & -4.50 & 3.81 & -2.94 \\
\hline 2 & 5.12 & -1.63 & 4.62 & -2.66 & 5.20 & -4.73 & --- & -- & 4.52 & -4.16 & 3.42 & -2.74 \\
\hline 3 & 4.78 & -2.73 & 5.23 & -3.55 & 5.12 & -4.99 & --- & --- & 4.00 & -4.07 & 2.82 & -2.85 \\
\hline 4 & 5.26 & -2.45 & 5.57 & -4.29 & 5.35 & -5.01 & --- & --- & 3.55 & -4.07 & 3.50 & -2.39 \\
\hline 5 & 5.53 & -2.90 & 5.71 & -4.73 & 5.33 & -4.75 & --- & --- & 2.98 & -3.67 & 3.36 & -2.35 \\
\hline 6 & 5.76 & -3.83 & 5.77 & -4.95 & --- & --- & --- & -- & 2.73 & -3.11 & 2.64 & -2.95 \\
\hline 7 & 5.61 & -4.53 & --- & --- & --- & --- & --- & --- & 3.06 & -2.55 & --- & --- \\
\hline 8 & 5.51 & -4.77 & --- & --- & 3.50 & -4.26 & --- & -- & 3.06 & -2.59 & --- & --- \\
\hline 9 & 5.42 & -4.61 & 4.70 & -4.33 & 2.66 & -3.87 & --- & --- & 2.48 & -2.65 & --- & --- \\
\hline 10 & 5.24 & -4.20 & 4.15 & -3.79 & 2.74 & -3.13 & --- & --- & 2.12 & -2.97 & 3.10 & -2.66 \\
\hline 11 & 4.32 & -4.06 & 3.29 & -3.40 & 2.82 & -2.52 & --- & --- & 2.79 & -3.52 & --- & --- \\
\hline 12 & 3.41 & -3.48 & 2.48 & -3.18 & 3.05 & -1.91 & --- & -- & 2.47 & -3.71 & --- & --- \\
\hline 13 & 3.05 & -3.11 & 2.69 & -2.83 & 3.50 & -2.20 & --- & --- & 2.68 & -4.21 & --- & --- \\
\hline 14 & 2.47 & -3.09 & 2.96 & -2.36 & 3.74 & -2.53 & --- & --- & 3.57 & -4.66 & --- & --- \\
\hline 15 & 2.70 & -3.07 & 3.09 & -2.02 & 3.91 & -2.93 & --- & -- & 3.78 & -4.26 & 4.00 & -3.60 \\
\hline 16 & 3.12 & -2.88 & 3.49 & -2.62 & 3.94 & -3.65 & --- & -- & 3.65 & -4.36 & 4.90 & -3.12 \\
\hline 17 & 3.48 & -2.46 & 4.20 & -2.97 & 4.38 & -4.17 & --- & --- & 3.63 & -4.32 & 5.16 & -3.39 \\
\hline 18 & 3.81 & -2.53 & 4.14 & -2.94 & 4.56 & -4.14 & -- & --- & 4.07 & -4.15 & 5.10 & -3.75 \\
\hline 19 & 4.08 & -2.83 & 3.97 & -3.93 & --- & --- & --- & -- & 4.05 & -3.80 & 4.35 & -3.85 \\
\hline 20 & 4.30 & -2.83 & 3.97 & -4.35 & --- & --- & -- & --- & 4.56 & -2.97 & 3.79 & -3.58 \\
\hline 21 & 4.34 & -2.97 & 4.08 & -4.46 & -- & --- & --- & -- & 4.79 & -2.80 & --- & --- \\
\hline 22 & 4.69 & -3.35 & 4.35 & -4.41 & --- & --- & --- & --- & 4.85 & -2.83 & --- & --- \\
\hline 23 & 4.76 & -3.51 & 4.58 & -4.01 & 3.93 & -4.07 & -- & --- & 4.52 & -2.89 & $-\therefore-$ & --- \\
\hline 24 & 4.61 & -3.51 & 4.61 & -4.00 & 3.55 & -3.68 & --- & --- & 4.15 & -3.12 & 3.11 & -2.65 \\
\hline 25 & 4.59 & -3.28 & 3.78 & -4.19 & 3.90 & -3.15 & --- & --- & 3.92 & -3.31 & 3.00 & -2.62 \\
\hline 26 & 4.24 & -3.33 & 3.39 & -3.88 & 4.28 & -2.22 & --- & -- & 3.73 & -3.51 & 3.07 & -2.71 \\
\hline 27 & 3.47 & -3.51 & 3.21 & -3.50 & 4.53 & -2.32 & --- & --- & 3.67 & -3.86 & 3.38 & -2.59 \\
\hline 28 & 3.02 & -3.27 & 3.49 & -2.90 & 5.10 & -2.87 & --- & --- & 3.83 & -4.01 & 3.68 & -2.29 \\
\hline 29 & 2.98 & -3.12 & 3.72 & -2.76 & 5.50 & -3.26 & 3.68 & -4.61 & 3.93 & -3.90 & 4.19 & -2.06 \\
\hline 30 & 3.74 & -2.92 & 4.11 & -2.76 & 5.45 & -3.94 & 3.93 & -4.63 & 3.92 & -3.74 & 4.46 & -2.53 \\
\hline 31 & -- & --- & 4.55 & -3.73 & --- & --- & 4.25 & -4.72 & 3.93 & -3.36 & --- & --- \\
\hline MONTH & 5.76 & -4.77 & --- & --- & --- & --- & --- & --- & 4.85 & -4.66 & --- & --- \\
\hline
\end{tabular}


Table 8.--Maximum and minimum daily water levels for the Alsea River at Waldport--Continued

GAGE HEIGHT, IN FEET, WATER YEAR OCTOBER 1988 TO SEPTEMBER 1989

\begin{tabular}{|c|c|c|c|c|c|c|c|c|c|c|c|c|}
\hline \multirow[b]{2}{*}{ DAY } & \multicolumn{2}{|c|}{ OCTOBER } & \multicolumn{2}{|c|}{ NOVEMBER } & \multicolumn{2}{|c|}{ DECEMBER } & \multicolumn{2}{|c|}{ JANUARY } & \multicolumn{2}{|c|}{ FEBRUARY } & \multicolumn{2}{|c|}{ MARCH } \\
\hline & MAX & MIN & $\operatorname{MAX}$ & MIN & MAX & MIN & MAX & MIN & MAX & MIN & MAX & MIN \\
\hline 1 & 3.88 & -3.29 & 3.32 & -2.32 & 3.83 & -1.74 & 4.26 & -1.99 & 4.57 & -2.41 & 3.20 & -2.56 \\
\hline 2 & 3.11 & -2.97 & 4.24 & -1.53 & 4.20 & -1.11 & 4.26 & -2.61 & 4.29 & -3.37 & 3.59 & -2.84 \\
\hline 3 & 2.90 & -3.03 & 4.46 & -.65 & 3.75 & -1.53 & 4.91 & -2.74 & 4.01 & -4.55 & 3.37 & -3.87 \\
\hline 4 & 2.71 & -2.86 & 4.11 & -.86 & 4.39 & -1.97 & 5.42 & -3.55 & 4.18 & -5.20 & 3.94 & -3.69 \\
\hline 5 & 2.82 & -2.57 & 5.01 & -.72 & 4.56 & -2.66 & 5.24 & -4.27 & 4.01 & -5.23 & 5.54 & -2.48 \\
\hline 6 & 3.08 & -2.74 & 4.50 & -2.10 & 4.66 & -3.59 & 5.33 & -4.60 & 4.58 & -5.23 & 5.72 & -3.54 \\
\hline 7 & 3.38 & -2.22 & 4.61 & -2.92 & 4.36 & -4.35 & 6.12 & -4.68 & 4.68 & -4.72 & 5.41 & -3.37 \\
\hline 8 & 3.25 & -2.51 & 5.09 & -3.01 & 4.57 & -4.87 & 6.21 & -3.87 & 4.57 & -4.10 & 5.43 & -3.38 \\
\hline 9 & 3.43 & -2.69 & 5.46 & -3.12 & 4.85 & -4.84 & 5.49 & -3.14 & 4.63 & -3.49 & 5.70 & -2.85 \\
\hline 10 & 3.95 & -2.98 & 5.66 & -3.62 & 4.93 & -5.00 & 4.61 & -2.84 & 4.76 & -3.09 & 6.25 & -3.39 \\
\hline 11 & 3.91 & -3.59 & 5.74 & -3.22 & 4.81 & -4.73 & 3.98 & -3.67 & 4.63 & -3.25 & 6.25 & -2.92 \\
\hline 12 & 4.36 & -3.33 & 5.52 & -3.12 & 5.12 & -4.27 & 5.60 & -2.43 & 4.50 & -3.28 & 6.17 & -2.86 \\
\hline 13 & 4.93 & -3.07 & 5.81 & -3.17 & 3.99 & -4.32 & 5.45 & -1.46 & 4.08 & -3.19 & 5.38 & -2.20 \\
\hline 14 & 4.58 & -3.54 & 4.96 & -2.54 & 3.01 & -4.39 & 5.80 & -1.39 & 3.85 & -3.58 & 4.78 & -2.67 \\
\hline 15 & 3.96 & -3.44 & 4.11 & -2.57 & 2.89 & -3.84 & 5.01 & -1.71 & 3.45 & -4.02 & 4.08 & -2.32 \\
\hline 16 & 3.89 & -3.45 & 4.00 & -2.21 & 3.28 & -3.49 & 5.49 & -2.00 & 4.69 & -3.28 & 4.02 & -2.13 \\
\hline 17 & 3.24 & -3.41 & 3.92 & -2.22 & 4.10 & -2.63 & 4.99 & -2.52 & 4.58 & -3.07 & 4.09 & -2.27 \\
\hline 18 & 3.03 & -3.21 & 4.14 & -2.36 & 4.47 & -3.05 & 4.96 & -4.48 & 4.91 & -3.17 & 4.11 & -2.60 \\
\hline 19 & 2.80 & -3.39 & 4.61 & -2.30 & 5.27 & -3.68 & 4.88 & -3.90 & 4.76 & -3.32 & 3.43 & -3.18 \\
\hline 20 & 2.87 & -3.54 & 5.97 & -2.69 & 6.13 & -3.22 & 4.98 & -3.91 & 4.48 & -3.44 & 3.79 & -2.97 \\
\hline 21 & 3.24 & -3.36 & 7.09 & -3.03 & 7.72 & -2.99 & 5.61 & -3.68 & 4.12 & -2.86 & 3.92 & -2.78 \\
\hline 22 & 3.90 & -3.33 & 8.18 & -1.87 & 6.76 & -3.45 & 4.97 & -3.99 & 4.45 & -2.24 & 3.89 & -2.29 \\
\hline 23 & 4.76 & -3.54 & 7.92 & -2.58 & 6.39 & -3.47 & 4.27 & -4.15 & 4.46 & -2.07 & 4.04 & -2.97 \\
\hline 24 & 5.65 & -3.89 & 7.92 & -2.27 & 6.16 & -3.77 & 4.30 & -3.37 & 4.55 & -1.35 & 4.11 & -3.19 \\
\hline 25 & 5.79 & -4.50 & 6.97 & -2.75 & 5.41 & -3.82 & 3.37 & -3.26 & 4.34 & -2.08 & 4.44 & -2.94 \\
\hline 26 & 5.38 & -4.87 & 5.58 & -3.49 & 4.00 & -4.02 & 3.76 & -2.55 & 3.69 & -2.74 & 4.68 & -2.39 \\
\hline 27 & 5.55 & -4.49 & 5.29 & -2.35 & 4.06 & -2.82 & 3.87 & -2.29 & 3.06 & -2.90 & 5.04 & -2.38 \\
\hline 28 & 4.95 & -4.35 & 4.52 & -2.57 & 3.68 & -2.77 & 3.18 & -1.89 & 3.14 & -2.76 & 5.42 & -1.79 \\
\hline 29 & 4.22 & -4.05 & 3.24 & -2.73 & 3.51 & -1.15 & 3.22 & -1.91 & --- & --- & 4.43 & -2.30 \\
\hline 30 & 3.68 & -3.14 & 3.16 & -2.24 & 5.19 & -.34 & 3.59 & -1.94 & -- & --- & 3.72 & -2.26 \\
\hline 31 & 3.08 & -2.45 & --- & --- & 4.26 & -1.55 & 3.90 & -2.22 & -- & --- & 4.15 & -1.80 \\
\hline MON & 5.79 & -4.87 & 8.18 & -3.62 & 7.72 & -5.00 & 6.21 & -4.68 & 4.91 & -5.23 & 6.25 & -3.87 \\
\hline
\end{tabular}


Table 8.--Maximum and minimum daily water levels for Alsea River at Waldport--Continued

GAGE HEIGHT,. IN FEET, WATER YEAR OCTOBER 1989 TO SEPTEMBER 1990

\begin{tabular}{|c|c|c|c|c|c|c|c|c|c|c|c|c|}
\hline \multirow[b]{2}{*}{ DAY } & \multicolumn{2}{|c|}{ OCTOBER } & \multicolumn{2}{|c|}{ NOVEMBER } & \multicolumn{2}{|c|}{ DECEMBER } & \multicolumn{2}{|c|}{ JANUARY } & \multicolumn{2}{|c|}{ FEBRUARY } & \multicolumn{2}{|c|}{ MARCH } \\
\hline & MAX & MIN & MAX & MIN & $\operatorname{MAX}$ & MIN & MAX & MIN & MAX & MIN & MAX & MIN \\
\hline 1 & 4.46 & -2.70 & --- & --- & --- & --- & --- & --- & -- & --- & --- & -- \\
\hline 2 & 4.29 & -3.12 & 4.06 & -3.55 & -- & --- & -- & --- & -- & -- & 5.06 & -2.96 \\
\hline 3 & 3.92 & -3.22 & 4.02 & -2.87 & --- & -- & -- & --- & $-\infty$ & --- & 5.03 & -2.57 \\
\hline 4 & 3.79 & -2.99 & 3.66 & -2.84 & -- & -- & 4.93 & -1.39 & -- & -- & 4.87 & -2.47 \\
\hline 5 & 3.78 & -2.65 & 2.94 & -2.85 & --- & -- & 4.99 & -1.93 & $-\infty$ & -- & 4.70 & -2.55 \\
\hline 6 & 3.22 & -2.90 & 2.58 & -2.53 & -- & --- & 5.89 & -1.24 & $-\infty$ & -- & 4.50 & -2.05 \\
\hline 7 & 2.75 & -2.88 & 2.81 & -2.45 & -- & $-\infty$ & 6.90 & -.87 & $-\infty$ & -- & 5.10 & -2.56 \\
\hline 8 & 2.66 & -2.81 & 3.19 & -2.57 & -- & --- & $-\infty$ & $-\infty$ & -- & --- & 4.77 & -2.51 \\
\hline 9 & 2.71 & -2.80 & 4.07 & -2.24 & -- & -- & $-\infty$ & $-\infty$ & -- & -- & 4.94 & -1.92 \\
\hline 10 & 2.92 & -3.25 & 4.53 & -3.22 & -- & -- & -- & -- & -- & -- & 5.11 & -1.72 \\
\hline 11 & 3.14 & -3.20 & 5.01 & -3.95 & -- & --- & -- & -- & -- & -- & 5.55 & -1.47 \\
\hline 12 & 4.04 & -3.10 & 5.70 & -4.48 & 6.05 & -4.97 & -- & $-\infty$ & -- & $-\infty$ & 4.92 & -1.95 \\
\hline 13 & 4.73 & -3.27 & 5.48 & -4.77 & 5.65 & -5.26 & --- & -- & -- & $--\infty$ & 4.30 & -2.42 \\
\hline 14 & 5.15 & -4.15 & -- & -- & 5.23 & -5.22 & -- & -- & $--\infty$ & -- & 5.17 & -1.78 \\
\hline 15 & 5.28 & -4.83 & --- & --- & 4.75 & -4.72 & -- & -- & -- & -- & 4.30 & -2.57 \\
\hline 16 & 5.35 & -5.19 & 4.93 & -4.80 & 4.36 & -3.96 & -- & $-\infty$ & 5.15 & -.16 & 4.01 & -2.42 \\
\hline 17 & 5.11 & -4.97 & 4.36 & -4.08 & 3.81 & -3.06 & -- & -- & 4.38 & -.97 & 4.15 & -1.70 \\
\hline 18 & 4.56 & -4.49 & 3.60 & -3.61 & 3.89 & -2.79 & $-\infty$ & -- & 3.55 & -2.01 & 3.63 & -2.31 \\
\hline 19 & 4.90 & -3.38 & 2.78 & -2.80 & 3.34 & -2.22 & $-\infty$ & --- & 3.20 & -2.42 & 3.37 & -1.89 \\
\hline 20 & 4.52 & -3.12 & 3.18 & -2.61 & 3.46 & -1.92 & $-\infty$ & -- & 3.88 & -1.76 & 3.10 & -2.54 \\
\hline 21 & 3.89 & -2.61 & 3.08 & -2.29 & 3.69 & -1.87 & -- & $=$ & 3.86 & -3.34 & 3.18 & -2.90 \\
\hline 22 & 4.27 & -1.90 & 3.41 & -1.76 & 3.80 & -2.42 & -- & --- & 4.05 & -3.59 & 3.22 & -2.84 \\
\hline 23 & --- & --- & 4.48 & -1.53 & 3.97 & -3.00 & $-\infty$ & $\ldots$ & 4.42 & -3.80 & 3.42 & -3.49 \\
\hline 24 & --- & --- & 4.82 & -1.47 & 4.07 & -3.63 & - - & -- & 4.34 & -3.98 & 3.57 & -3.59 \\
\hline 25 & --- & --- & 5.44 & -1.79 & 4.18 & -3.58 & -- & -- & 4.30 & -3.78 & 4.36 & -3.00 \\
\hline 26 & --- & --- & 5.18 & -2.74 & 5.31 & -3.53 & $\ldots$ & -- & 4.28 & -3.34 & 4.29 & -3.20 \\
\hline 27 & --- & --- & 4.52 & -3.56 & 5.33 & -3.77 & $-\infty$ & $-\infty$ & 4.55 & -2.76 & 4.36 & -3.92 \\
\hline 28 & --- & --- & 4.59 & -3.91 & 4.88 & -4.45 & $\ldots$ & 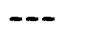 & 4.80 & -3.07 & 4.74 & -4.57 \\
\hline 29 & --- & --- & --- & --- & 4.21 & -4.94 & -- & 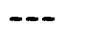 & $\ldots$ & -- & 4.85 & -4.65 \\
\hline 30 & --- & --- & --- & --- & 4.33 & -4.31 & $=$ & 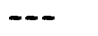 & $-\infty$ & $-\infty$ & 4.90 & -4.73 \\
\hline 31 & --- & --- & --- & --- & 4.44 & -3.07 & -- & $\cdots$ & $\ldots$ & $-\cdots$ & 5.08 & -3.88 \\
\hline MONTH & --- & --- & --- & --- & -- & --- & -- & --- & -- & $-\infty$ & --- & -- \\
\hline
\end{tabular}


Table 8.--Maximum and minimum daily water levels for Alsea River at Waldport--Continued GAGE HEIGHT, IN FEET, WATER YEAR OCTOBER 1989 TO SEPTEMBER 1990

\begin{tabular}{|c|c|c|c|c|c|c|c|c|c|c|c|c|}
\hline \multirow[b]{2}{*}{ DAY } & \multicolumn{2}{|c|}{ APRIL } & \multicolumn{2}{|c|}{ MAY } & \multicolumn{2}{|c|}{ JUNE } & \multicolumn{2}{|c|}{ JULY } & \multicolumn{2}{|c|}{ AUGUST } & \multicolumn{2}{|c|}{ SEPTEMBER } \\
\hline & $\operatorname{MAX}$ & MIN & $\operatorname{MAX}$ & MIN & $\operatorname{MAX}$ & MIN & $\operatorname{MAX}$ & MIN & $\operatorname{MAX}$ & MIN & $\operatorname{MAX}$ & MIN \\
\hline 1 & 4.48 & -3.72 & 2.80 & -4.06 & 4.21 & -1.19 & 3.30 & -2.34 & --- & --- & -- & -- \\
\hline 2 & 3.90 & -3.42 & 2.34 & -3.75 & 4.24 & -1.41 & 3.32 & -2.82 & --- & --- & --- & --- \\
\hline 3 & 3.27 & -3.58 & 3.21 & -3.43 & 4.47 & -2.03 & 3.55 & -3.25 & -- & --- & --- & --- \\
\hline 4 & 2.91 & -3.52 & 3.04 & -2.81 & 4.38 & -2.59 & 3.81 & -3.37 & $-\cdots$ & --- & --- & -- \\
\hline 5 & 3.41 & -3.00 & 3.40 & -2.28 & 4.47 & -3.15 & 4.28 & -3.22 & -- & --- & --- & --- \\
\hline 6 & 3.49 & -2.82 & 3.64 & -2.81 & 4.28 & -3.75 & 4.26 & -3.78 & -- & -- & --- & -- \\
\hline 7 & 3.72 & -2.39 & 3.44 & -3.45 & 4.21 & -3.77 & --- & --- & -- & -- & --- & --- \\
\hline 8 & 3.74 & -2.48 & 3.50 & -3.99 & 4.44 & -3.89 & --- & --- & -- & -- & --- & -- \\
\hline 9. & 3.77 & -3.11 & 3.64 & -3.97 & 4.24 & -4.13 & -- & -- & $\ldots$ & -- & --- & -- \\
\hline 10 & 3.76 & -3.12 & 3.99 & -4.09 & 4.41 & -4.13 & --- & --- & --- & --- & --- & -- \\
\hline 11 & 4.51 & -3.21 & 4.08 & -4.20 & 3.81 & -3.84 & --- & --- & --- & --- & --- & -- \\
\hline 12 & 3.93 & -3.84 & 3.80 & -4.24 & 3.77 & -3.59 & -- & --- & --- & -- & --- & --- \\
\hline 13 & 3.79 & -3.71 & 3.50 & -4.30 & 3.18 & -3.17 & --- & --- & -- & -- & --- & --- \\
\hline 14 & 3.80 & -3.55 & 3.26 & -4.09 & 2.79 & -3.11 & -- & -- & -- & -- & --- & --- \\
\hline 15 & 3.67 & -3.00 & 3.05 & -3.78 & 3.02 & -2.89 & --- & --- & --- & -- & --- & --- \\
\hline 16 & 3.49 & -2.65 & 2.82 & -3.64 & 3.61 & -2.51 & --- & --- & -- & $-\cdots$ & --- & -- \\
\hline 17 & 3.06 & -2.68 & 2.02 & -3.55 & 3.84 & -3.19 & --- & -- & --- & -- & --- & -- \\
\hline 18 & 2.67 & -2.59 & 2.62 & -3.19 & 3.83 & -4.37 & --- & --- & --- & -- & --- & --- \\
\hline 19 & 3.15 & -2.40 & 4.43 & -2.07 & 4.23 & -4.96 & --- & --- & --- & -- & --- & --- \\
\hline 20 & 3.45 & -2.44 & 4.54 & -1.21 & 4.76 & -5.17 & -- & --- & -- & -- & -- & --- \\
\hline 21 & 3.82 & -2.44 & 5.51 & -2.24 & 5.38 & -4.64 & --- & -- & --- & --- & --- & --- \\
\hline 22 & 5.06 & -1.65 & 5.26 & -3.45 & 5.88 & -4.87 & --- & --- & --- & --- & --- & --- \\
\hline 23 & 5.04 & -2.28 & 5.64 & -4.14 & 5.19 & -5.06 & --- & -- & --- & -- & --- & --- \\
\hline 24 & 5.20 & -3.76 & 5.56 & -4.75 & 4.63 & -5.19 & --- & --- & --- & --- & --- & --- \\
\hline 25 & 5.46 & -3.98 & 5.53 & -5.03 & 3.98 & -5.27 & --- & --- & -- & -- & --- & --- \\
\hline 26 & 5.77 & -4.60 & 5.56 & -4.60 & 3.73 & -5.11 & -- & -- & --- & --- & --- & --- \\
\hline 27 & 5.59 & -4.27 & 5.76 & -4.03 & 3.48 & -4.07 & --- & --- & -- & -- & -- & --- \\
\hline 28 & 5.72 & -3.56 & 5.09 & -3.74 & 3.49 & -2.98 & --- & --- & --- & --- & --- & --- \\
\hline 29 & 4.64 & -4.36 & 4.41 & -3.27 & 3.55 & -2.37 & --- & -- & --- & --- & -- & --- \\
\hline 30 & 3.58 & -4.51 & 4.74 & -2.12 & 3.26 & -1.66 & --- & -- & --- & --- & --- & --- \\
\hline 31 & --- & --- & 4.02 & -1.11 & -- & --- & --- & -- & -- & -- & $\cdots$ & --- \\
\hline MON & 5.77 & -4.60 & 5.76 & -5.03 & 5.88 & -5.27 & -- & $\cdots$ & $-\cdots$ & -- & -- & --- \\
\hline
\end{tabular}


Table 9.--Maximum and minimum daily water levels for Alsea River at Drift Creek near Waldport, river mile 5.0.

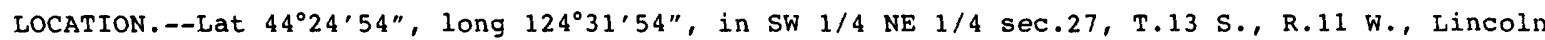
County, Hydrologic Unit 17100205, on left bank, 4 miles east of waldport, and at mile 5.0. PERIOD OF RECORD.--April 1989 to July 1990 (gage height).

GAGE.--Water-stage recorder. Datum of gage is 0.00 feet above National Geodetic Vertical Datum of 1929 (levels by Oregon Department of Transportation).

REMARKS.--Site is affected by tidal fluctuations.

EXTREMES FOR PERIOD OF RECORD.--Maximum gage height April 23, 1989 to July 11, 1990, 8.47 feet January 9, 1990; minimum gage height -2.99 feet June $21,1989$.

GAGE HEIGHT, FEET, WATER YEAR OCTOBER 1988 TO SEPTEMBER 1989

\begin{tabular}{|c|c|c|c|c|c|c|c|c|c|c|c|c|}
\hline \multirow[b]{2}{*}{ DAY } & \multicolumn{2}{|c|}{ APRIL } & \multicolumn{2}{|c|}{ MAY } & \multicolumn{2}{|c|}{ JUNE } & \multicolumn{2}{|c|}{ JULY } & \multicolumn{2}{|c|}{ AUGUST } & \multicolumn{2}{|c|}{ SEPTEMBER } \\
\hline & MAX & MIN & MAX & MIN & $\operatorname{MAX}$ & MIN & MAX & MIN & $\operatorname{MAX}$ & MIN & MAX & MIN \\
\hline 1 & -- & -- & 4.21 & -1.98 & 5.14 & -2.65 & 5.57 & -2.68 & 4.78 & -2.76 & 3.85 & -2.37 \\
\hline 2 & --- & --- & 4.84 & -1.89 & 5.29 & -2.76 & 5.38 & -2.76 & 5.08 & -2.44 & 3.55 & -2.28 \\
\hline 3 & -- & -- & 5.33 & -2.32 & 5.34 & -2.83 & 5.09 & -2.79 & 4.48 & -2.51 & 3.47 & -2.224 \\
\hline 4 & -- & --- & 5.39 & -2.49 & 5.46 & -2.82 & 4.65 & -2.84 & 3.77 & -2.58 & 3.82 & -2.00 \\
\hline 5 & --- & --- & 5.86 & -2.59 & 5.47 & -2.77 & 4.28 & -2.91 & 3.40 & -2.50 & 3.86 & -2.05 \\
\hline 6 & --- & -- & 5.95 & -2.66 & 5.18 & -2.79 & 3.57 & -2.91 & 3.46 & -2.25 & 3.64 & -2.06 \\
\hline 7 & $\ldots$ & -- & 5.63 & -2.76 & 4.35 & -2.87 & 3.25 & -2.77 & 3.39 & -1.92 & 3.44 & -2.22 \\
\hline 8 & -- & --- & 5.16 & -2.70 & 3.62 & -2.76 & 2.94 & -2.67 & 3.37 & -1.91 & 3.34 & -2.23 \\
\hline 9 & --- & --- & 4.84 & -2.64 & 2.80 & -2.73 & 1.85 & -2.36 & 3.32 & -1.95 & 3.23 & -2.25 \\
\hline 10 & --- & --- & 4.24 & -2.59 & 2.89 & -2.49 & 3.07 & -2.36 & 3.05 & -2.17 & 3.46 & -2.46 \\
\hline 11 & --- & --- & 3.38 & -2.51 & 2.98 & -2.27 & 3.22 & -1.84 & 3.18 & -2.55 & 3.74 & -2.61 \\
\hline 12 & -- & -- & 2.59 & -2.46 & 3.15 & -1.81 & 3.28 & -2.10 & 3.44 & -2.64 & 4.22 & -2.73 \\
\hline 13 & -- & -- & 2.85 & -2.25 & 3.61 & -1.92 & 3.24 & -2.44 & 3.68 & -2.88 & 4.57 & -2.65 \\
\hline 14 & --- & --- & 3.06 & -1.97 & 3.82 & -2.12 & 3.39 & -2.67 & 3.95 & -2.94 & 4.48 & -2.54 \\
\hline 15 & --- & --- & 3.25 & -1.71 & 4.00 & -2.30 & 3.59 & -2.80 & 4.31 & -2.98 & 4.69 & -2.40 \\
\hline 16 & -- & -- & 3.51 & $-2 \cdot 10$ & 4.03 & -2.70 & 4.10 & -2.76 & 4.38 & -2.92 & 5.37 & -2.21 \\
\hline 17 & --- & --- & 3.96 & -2.25 & 4.49 & -2.85 & 4.61 & -2.74 & 4.52 & -2.84 & 5.74 & -2.34 \\
\hline 18 & --- & --- & 4.24 & -2.21 & 4.58 & -2.82 & 5.05 & -2.70 & 4.45 & -2.72 & 5.77 & -2.52 \\
\hline 19 & --- & --- & 4.15 & -2.58 & 4.66 & -2.73 & 4.88 & -2.85 & 4.35 & -2.58 & 5.23 & -2.43 \\
\hline 20 & --- & --- & 4.09 & -2.76 & 4.88 & -2.82 & 4.71 & -2.84 & 4.85 & -2.31 & 4.70 & -2.64 \\
\hline 21 & --- & --- & 4.24 & -2.79 & 4.27 & -2.99 & 4.15 & -2.85 & 5.05 & -2.01 & 4.36 & -2.67 \\
\hline 22 & --- & --- & 4.50 & -2.78 & 3.99 & -2.95 & 3.79 & -2.78 & 4.99 & -2.23 & 3.85 & -2.55 \\
\hline 23 & 4.88 & -2.25 & 4.66 & -2.64 & 3.98 & -2.74 & 3.91 & -2.57 & 4.47 & -2.33 & 3.74 & -2.42 \\
\hline 24 & --- & --- & 4.69 & -2.45 & 3.68 & -2.66 & 4.22 & -2.32 & 4.10 & -2.57 & 3.74 & -2.22 \\
\hline 25 & 4.74 & -.16 & 3.88 & -2.60 & 4.02 & -2.39 & 4.45 & -2.24 & 3.95 & -2.70 & 3.82 & -2.25 \\
\hline 26 & $4 \cdot 34$ & -2.32 & 3.47 & -2.60 & 4.47 & -1.89 & 4.39 & -2.34 & 3.89 & -2.71 & 3.76 & -2.27 \\
\hline 27 & 3.52 & -2.50 & 3.37 & -2.40 & 4.62 & -1.95 & 4.33 & -2.64 & 3.86 & -2.84 & 3.82 & -2.12 \\
\hline 28 & 3.07 & -2.46 & 3.59 & -2.13 & 5.21 & -2.24 & 4.51 & -2.82 & 4.13 & -2.82 & 4.03 & -1.95 \\
\hline 29 & 3.12 & -2.43 & 3.88 & -2.07 & 5.61 & -2.41 & 4.58 & -2.89 & 4.04 & -2.73 & 4.35 & -1.85 \\
\hline 30 & 3.72 & -2.13 & 4.34 & -2.04 & 5.54 & -2.57 & 4.62 & -2.93 & 4.26 & -2.62 & 4.36 & -2.14 \\
\hline 31 & -- & -- & 4.69 & -2.45 & -- & --- & 4.69 & -2.88 & 4.11 & -2.53 & --- & --- \\
\hline MONT & ГH --- & -- & 5.95 & -2.79 & 5.61 & -2.99 & 5.57 & -2.93 & 5.08 & -2.98 & 5.77 & -2.73 \\
\hline
\end{tabular}


Table 9.--Maximum and minimun daily water levels at Alsea River at Drift Creek near Waldport, river mile 5.0--Continued

GAGE HEIGHT, FEET, WATER YEAR OCTOBER 1989 TO SEPTEMBER 1990

\begin{tabular}{|c|c|c|c|c|c|c|c|c|c|c|c|c|}
\hline \multirow[b]{2}{*}{ DAY } & \multicolumn{2}{|c|}{ OCTOBER } & \multicolumn{2}{|c|}{ NOVEMBER } & \multicolumn{2}{|c|}{ DECEMBER } & \multicolumn{2}{|c|}{ JANUARY } & \multicolumn{2}{|c|}{ FEBRUARY } & \multicolumn{2}{|c|}{ MARCH } \\
\hline & $\operatorname{MAX}$ & MIN & MAX & MIN & MA & MIN & MA & MIN & MAX & MIN & MA & $\mathbf{M}$ \\
\hline 1 & 4.27 & -2.25 & 4.10 & -2.69 & 4.48 & -2.43 & 4.51 & -2.04 & 6.58 & 0.91 & 5.25 & -1.56 \\
\hline 2 & 4.16 & -2.49 & 4.23 & -2.52 & 4.78 & -1.96 & 4.04 & -2.01 & 6.70 & .45 & 5.35 & -1.61 \\
\hline 3 & 3.73 & -2.56 & 4.19 & -2.34 & 5.01 & -1.42 & 3.82 & -1.89 & 6.68 & .47 & 5.29 & -1.53 \\
\hline 4 & 3.70 & -2.46 & 3.82 & -2.30 & 4.88 & -.88 & 4.85 & -1.71 & 5.65 & .04 & 5.07 & -1.21 \\
\hline 5 & 3.70 & -2.44 & 3.22 & -2.23 & 4.55 & -.19 & 5.09 & -1.35 & 6.23 & .33 & 4.77 & -1.33 \\
\hline 6 & 3.21 & -2.38 & 2.88 & -2.40 & 4.32 & -.41 & 5.86 & -.20 & 6.85 & .11 & 4.52 & -1.26 \\
\hline 7 & 2.72 & -2.55 & 3.19 & -2.11 & 4.81 & -.86 & 6.80 & 2.01 & 6.26 & -.11 & 5.23 & -1.38 \\
\hline 8 & 2.64 & -2.53 & 3.56 & -2.14 & 5.59 & -.98 & 6.91 & 1.48 & 6.61 & 1.66 & 4.85 & -1.36 \\
\hline 9 & 2.81 & -2.52 & 4.31 & -1.77 & 5.67 & -1.58 & 8.47 & 1.02 & 6.30 & 1.58 & 5.11 & -1.12 \\
\hline 10 & 3.11 & -2.56 & 5.02 & -2.17 & 5.53 & -2.04 & 6.43 & .162 & 5.69 & 1.31 & 5.38 & -.32 \\
\hline 11 & 3.39 & -2.46 & 5.48 & -2.39 & 5.53 & -2.25 & 6.17 & -.22 & 5.15 & .37 & 5.25 & -.49 \\
\hline 12 & 4.31 & -2.29 & 6.11 & -2.19 & 5.94 & -2.31 & 6.08 & -.74 & 5.17 & .12 & 5.04 & -.88 \\
\hline 13 & 5.01 & -2.11 & 6.14 & -2.32 & 5.75 & -2.40 & 6.07 & -.98 & 5.07 & -.46 & 4.44 & -1.19 \\
\hline 14 & 5.41 & -2.43 & 6.52 & -2.49 & 5.42 & -2.46 & 4.85 & -1.26 & 4.14 & -.63 & 5.42 & -.86 \\
\hline 15 & 5.69 & -2.65 & 5.39 & -2.73 & 4.92 & -2.48 & 4.62 & -1.11 & 4.59 & .10 & 4.52 & -1.34 \\
\hline 16 & 5.72 & -2.78 & 4.74 & -2.73 & 4.47 & -2.34 & 5.16 & -.93 & 5.19 & .08 & 4.21 & -1.38 \\
\hline 17 & 5.63 & -2.79 & 4.32 & -2.63 & 4.02 & -2.12 & 4.43 & -1.09 & 4.34 & -.47 & 4.27 & -1.16 \\
\hline 18 & 4.94 & -2.69 & 3.56 & -2.64 & 3.80 & -2.12 & 3.71 & $-1: 14$ & 3.38 & -1.11 & 3.61 & -1.56 \\
\hline 19 & 5.09 & -2.73 & 3.03 & -2.57 & 3.38 & -2.16 & 4.10 & -1.09 & 3.00 & -1.37 & 3.41 & -1.51 \\
\hline 20 & 4.60 & -2.39 & 3.41 & -2.30 & 3.56 & -1.84 & 4.01 & -1.35 & 3.90 & -1.13 & 3.05 & -1.81 \\
\hline 21 & 4.10 & -2.05 & 3.32 & -2.06 & 3.70 & -1.68 & 4.48 & -1.34 & 3.78 & -1.31 & 2.81 & -1.97 \\
\hline 22 & 4.36 & -1.71 & 3.69 & -1.71 & 3.82 & -1.96 & 4.80 & $-1,60$ & 4.15 & -1.11 & 3.23 & -1.99 \\
\hline 23 & 4.80 & -1.25 & 4.81 & -1.35 & 4.10 & -2.22 & 4.74 & $-1,68$ & 4.60 & -1.18 & 3.56 & -2.06 \\
\hline 24 & 4.40 & -1.35 & 5.15 & -1.26 & 4.08 & -2.50 & 4.61 & -2.00 & 4.60 & -1.29 & 3.86 & -2.10 \\
\hline 25 & 4.24 & -1.34 & 5.74 & -1.24 & 4.36 & -2.41 & 5.38 & -1.62 & 4.66 & -1.29 & 4.46 & -1.89 \\
\hline 26 & 4.69 & -1.40 & 5.51 & -1.59 & 5.10 & -2.35 & 5.66 & -1.25 & 4.67 & -1.29 & 4.50 & -1.94 \\
\hline 27 & 4.45 & -1.79 & 4.95 & -1.90 & 5.38 & -2.49 & 5.86 & -.86 & 4.95 & -1.26 & 4.65 & -2.26 \\
\hline 28 & 4.24 & -2.32 & 5.16 & -2.01 & 5.02 & -2.66 & 6.82 & .65 & 5.16 & -1.41 & 5.05 & -2.38 \\
\hline 29 & 4.11 & -2.47 & 5.06 & -2.19 & 4.38 & -2.80 & 6.11 & .83 & --- & --- & 5.20 & -2.44 \\
\hline 30 & 4.19 & -2.59 & 5.04 & -2.26 & 4.50 & -2.64 & 7.02 & 1.21 & --- & -- & 5.11 & -2.52 \\
\hline 31 & 4.29 & -2.66 & --- & -- & 4.85 & -2.22 & 6.56 & 1.00 & --- & --- & 5.09 & -2.42 \\
\hline MONT & 5.72 & -2.79 & 6.52 & -2.73 & 5.94 & -2.80 & 8.47 & -2.04 & 6.85 & -1.41 & 5.42 & -2.52 \\
\hline
\end{tabular}


Table 9.--Maximum and minimum daily water levels for Alsea River at Drift Creek near Waldport, river mile 5.0--Continued

GAGE HEIGHT, FEET, WATER YEAR OCTOBER 1989 TO SEPTEMBER 1990

\begin{tabular}{|c|c|c|c|c|c|c|c|c|c|c|c|c|}
\hline \multirow[b]{2}{*}{ DAY } & \multicolumn{2}{|c|}{ APRIL } & \multicolumn{2}{|c|}{ MAY } & \multicolumn{2}{|r|}{ JUNE } & \multicolumn{2}{|c|}{ JULY } & \multicolumn{2}{|c|}{ AUGUST } & \multicolumn{2}{|c|}{ SEPTEMBER } \\
\hline & $\operatorname{MAX}$ & MIN & MAX & MIN & MAX & MIN & MAX & MIN & MAX & MIN & MAX & MIN \\
\hline 1 & 4.78 & -2.41 & 2.86 & -2.13 & 4.17 & -1.11 & 3.62 & -2.01 & --- & --- & --- & --- \\
\hline 2 & 4.06 & -2.36 & 2.54 & -2.13 & 4.46 & -1.25 & 3.58 & -2.26 & --- & --- & --- & --- \\
\hline 3 & 3.26 & -2.42 & 2.80 & -2.11 & 4.53 & -1.30 & 3.90 & -2.51 & -- & --- & --- & --- \\
\hline 4 & 2.95 & -2.48 & 3.43 & -1.87 & 4.73 & -1.69 & 4.50 & -2.55 & -- & --- & --- & -- \\
\hline 5 & 3.30 & -2.13 & 3.62 & -1.67 & 4.80 & -1.94 & 4.59 & -2.43 & --- & --- & --- & --- \\
\hline 6 & 3.65 & -2.02 & 3.89 & -1.98 & 4.57 & -2.09 & 4.57 & -2.58 & --- & --- & --- & --- \\
\hline 7 & 3.91 & -1.92 & 3.74 & -2.28 & 4.49 & -2.16 & 4.57 & -2.71 & --- & --- & --- & --- \\
\hline 8 & 3.94 & -2.01 & 3.75 & -2.48 & 4.59 & -2.23 & 4.47 & -2.79 & --- & --- & --- & --- \\
\hline 9 & 3.94 & -2.27 & 3.83 & -2.49 & 4.75 & -2.30 & 4.27 & -2.83 & --- & --- & --- & --- \\
\hline 10 & 4.15 & -2.19 & 4.27 & -2.48 & 4.48 & -2.40 & 3.91 & -2.86 & --- & --- & --- & --- \\
\hline 11 & 4.60 & -2.24 & 4.23 & -2.55 & 4.31 & -2.43 & --- & --- & --- & --- & --- & --- \\
\hline 12 & 4.11 & -2.53 & 4.00 & -2.66 & 4.00 & -2.29 & --- & --- & --- & --- & --- & --- \\
\hline 13 & 4.00 & -2.49 & 3.61 & -2.71 & 3.70 & -2.28 & --- & --- & --- & --- & --- & --- \\
\hline 14 & 4.01 & -2.47 & 3.41 & -2.70 & 3.20 & -2.21 & --- & --- & --- & --- & --- & --- \\
\hline 15 & 3.75 & -2.34 & 3.11 & -2.69 & 3.27 & -2.20 & --- & --- & --- & --- & --- & --- \\
\hline 16 & 3.55 & -2.24 & 2.51 & -2.75 & 3.89 & -1.92 & --- & --- & --- & --- & --- & -- \\
\hline 17 & 3.20 & -2.24 & 2.23 & -2.65 & 4.24 & -1.64 & --- & --- & --- & --- & --- & --- \\
\hline 18 & 2.63 & -2.29 & 2.78 & -2.48 & 4.21 & -2.24 & --- & --- & --- & --- & --- & --- \\
\hline 19 & 3.01 & -2.12 & 4.57 & -1.93 & 4.67 & -2.67 & --- & --- & --- & --- & --- & -- \\
\hline 20 & 3.59 & -1.90 & 4.77 & -1.37 & 5.23 & -2.80 & --- & --- & --- & --- & --- & --- \\
\hline 21 & 4.05 & -1.91 & 5.80 & -1.85 & 5.93 & -2.79 & 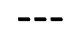 & --- & --- & --- & --- & --- \\
\hline 22 & 5.30 & -1.57 & 5.74 & -2.22 & 5.80 & -2.69 & --- & --- & --- & --- & --- & --- \\
\hline 23 & 5.33 & -1.62 & 5.93 & -2.46 & 6.14 & -2.70 & --- & --- & --- & --- & --- & --- \\
\hline 24 & 5.44 & -2.25 & 6.01 & -2.63 & 5.65 & -2.73 & --- & --- & --- & --- & --- & --- \\
\hline 25 & 5.76 & -2.26 & 5.94 & -2.72 & 4.99 & -2.78 & --- & -- & --- & --- & --- & --- \\
\hline 26 & 6.22 & -2.34 & 6.05 & -2.64 & 4.19 & -2.79 & --- & --- & --- & --- & -- & -- \\
\hline 27 & 6.14 & -1.26 & 6.23 & -2.39 & 3.68 & -2.67 & -- & --- & --- & -- & --- & -- \\
\hline 28 & 5.84 & -.08 & 5.45 & -2.26 & 3.78 & -2.30 & --- & -- & --- & -- & -- & -- \\
\hline 29 & 4.92 & -1.38 & 4.63 & -2.28 & 3.87 & -1.98 & -- & --- & --- & --- & --- & --- \\
\hline 30 & 3.70 & -1.90 & 4.94 & -1.65 & 3.64 & -1.60 & --- & -- & --- & -- & --- & -- \\
\hline 31 & --- & --- & 4.58 & -1.26 & -- & --- & --- & --- & --- & --- & --- & -- \\
\hline MONT & 6.22 & -2.53 & 6.23 & -2.75 & 6.14 & -2.80 & --- & --- & --- & --- & --- & --- \\
\hline
\end{tabular}


Table 10.--Maximum and minimum daily water levels for Alsea River at Kozy Kove near Waldport, river mile 11.2

LOCATION.--Lat $44^{\circ} 24^{\prime} 41^{\prime \prime}$, long $123^{\circ} 54^{\prime} 37^{\prime \prime}$, in NE 1/4 SW 1/4 sec.28, T.13 S., R.10 W., Lincoln County, Hydrologic Unit 17100205, on right bank, 10 miles east of waldport, and at mile 11.2 . PERIOD OF RECORD,--April 1989 to current year, (gage height).

GAGE.-Water-stage recorder. Datum of gage is 0.00 feet above National Geodetic Vertical Datum of 1929 (levels by Oregon Department of Transportation). April 21, 1989 to July 10, 1990.

REMARKS.--Record is poor. Site is affected by tides.

EXTREMES FOR PERIOD OF RECORD.--Maximum gage height, 11.23 feet Jan. 7, 1990; minimum gage height -3.24 feet July 28-30, Aug. 15, 1989 .

GAGE HEIGHT, FEET, WATER YEAR OCTOBER 1988 TO SEPTEMBER 1989

\begin{tabular}{|c|c|c|c|c|c|c|c|c|c|c|c|c|}
\hline \multirow[b]{2}{*}{ DAY } & \multicolumn{2}{|c|}{ APRIL } & \multicolumn{2}{|c|}{ MAY } & \multicolumn{2}{|c|}{ JUNE } & \multicolumn{2}{|c|}{ JULY } & \multicolumn{2}{|c|}{ AUGUST } & \multicolumn{2}{|c|}{ SEPTEMBER } \\
\hline & MAX & MIN & MAX & MIN & MAX & MIN & MAX & MIN & MAX & MIN & MAX & MIN \\
\hline 1 & --- & --- & 4.70 & -1.96 & 5.71 & -2.74 & 6.28 & -2.65 & 5.39 & -2.91 & 4.43 & -2.62 \\
\hline 2 & --- & --- & 5.32 & -1.91 & 5.83 & -2.84 & 6.21 & -2.72 & 5.67 & -2.51 & 4.23 & -2.51 \\
\hline 3 & --- & --- & 5.89 & -2.34 & 5.88 & -2.89 & 6.14 & -2.74 & 5.06 & -2.58 & 4.14 & -2.39 \\
\hline 4 & -- & --- & 6.32 & -2.47 & 6.01 & -2.91 & 5.88 & -2.77 & 4.35 & -2.71 & 4.50 & -2.14 \\
\hline 5 & --- & -- & 6.37 & -2.53 & 6.07 & -2.77 & 5.19 & -2.84 & 4.11 & -2.63 & 4.48 & -2.19 \\
\hline 6 & --- & --- & 6.10 & -2.60 & 5.79 & -2.79 & 4.44 & -2.85 & 4.17 & -2.39 & 4.16 & $-2 \cdot 34$ \\
\hline 7 & --- & --- & 5.77 & -2.65 & 5.03 & -2.77 & 3.97 & -2.72 & 4.01 & -2.06 & 3.95 & -2.31 \\
\hline 8 & --- & --- & 5.49 & -2.61 & 4.24 & -2.68 & 3.63 & -2.65 & 3.94 & -2.03 & 3.85 & -2.31 \\
\hline 9 & --- & --- & 5.18 & -2.57 & 3.41 & -2.72 & 3.64 & -2.33 & 3.74 & -2.10 & 3.77 & -2.53 \\
\hline 10 & --- & --- & 4.53 & -2.52 & 3.47 & -2.38 & 3.76 & -1.87 & 3.38 & -2.32 & 3.99 & -2.70 \\
\hline 11 & --- & --- & 3.63 & -2.49 & 3.59 & -2.22 & 3.80 & -1.84 & 3.60 & -2.71 & 4.24 & -2.85 \\
\hline 12 & --- & --- & 2.82 & -2.49 & 3.70 & -1.77 & 3.73 & -2.17 & 3.84 & -2.85 & 4.80 & -2.85 \\
\hline 13 & --- & --- & 3.10 & -2.26 & 4.19 & -1.96 & 3.83 & -2.52 & 4.19 & -3.09 & 5.28 & -2.64 \\
\hline 14 & --- & --- & 1.86 & -1.97 & 4.42 & -2.10 & 4.10 & -2.77 & 4.46 & -3.14 & 5.51 & -2.53 \\
\hline 15 & -- & --- & 3.36 & -1.79 & 4.65 & -2.27 & 4.53 & -2.92 & 4.76 & -3.24 & 5.62 & -2.26 \\
\hline 16 & -- & --- & 3.55 & -2.13 & 4.71 & -2.70 & 5.11 & -2.89 & 4.86 & -3.13 & 6.28 & -2.22 \\
\hline 17 & --- & --- & 3.89 & -2.30 & 5.17 & -2.86 & 5.45 & -2.89 & 5.15 & -3.05 & 6.61 & -2.36 \\
\hline 18 & -- & --- & 4.53 & -2.20 & 5.17 & -2.74 & 5.57 & -2.87 & 5.13 & -2.92 & 6.56 & -2.58 \\
\hline 19 & -- & --- & 4.50 & -2.55 & 5.43 & -2.67 & 5.39 & -3.04 & 5.25 & -2.70 & 6.06 & -2.62 \\
\hline 20 & --- & --- & 4.44 & -2.72 & 5.57 & -2.74 & 5.19 & -3.13 & 5.66 & -2.50 & 5.52 & -2.65 \\
\hline 21 & -- & --- & 4.55 & -2.77 & 4.94 & -2.88 & 4.56 & -3.17 & 5.71 & -2.43 & 5.12 & -2.49 \\
\hline 22 & 5.10 & -2.15 & 4.79 & -2.77 & 4.72 & -2.84 & 4.27 & -3.09 & 5.52 & -2.43 & 4.59 & -2.34 \\
\hline 23 & 5.17 & -2.20 & --- & $--\infty$ & 4.67 & -2.75 & 4.33 & -2.99 & 5.00 & -2.50 & 4.44 & -2.09 \\
\hline 24 & 5.02 & -2.12 & 5.05 & -2.55 & 4.58 & -2.68 & 4.50 & -2.80 & 4.54 & -2.73 & 4.53 & -2.14 \\
\hline 25 & 5.00 & -2.15 & 4.18 & -2.69 & 4.77 & -2.34 & 4.73 & -2.77 & 4.40 & -2.89 & 4.62 & -2.19 \\
\hline 26 & 4.57 & -2.28 & 3.72 & -2.75 & 5.24 & -1.89 & 4.58 & -2.88 & 4.32 & -2.91 & 4.75 & -2.08 \\
\hline 27 & 3.79 & -2.39 & 3.67 & -2.49 & 5.36 & -1.96 & 4.54 & -3.17 & 4.36 & -3.02 & 5.01 & -1.94 \\
\hline 28 & 3.27 & -2.39 & 3.95 & -2.24 & 5.84 & -2.24 & 4.81 & -3.24 & 4.65 & -3.02 & 5.23 & -1.71 \\
\hline 29 & 3.47 & -2.43 & 4.26 & -2.14 & 6.24 & -2.44 & 4.90 & -3.24 & 4.52 & -2.91 & 5.24 & -1.82 \\
\hline 30 & 4.18 & -2.10 & 4.69 & -2.18 & 6.14 & -2.55 & 5.18 & -3.24 & 4.76 & -2.85 & 5.18 & -2.11 \\
\hline 31 & --- & --- & 5.20 & -2.57 & --- & --- & 5.01 & -3.09 & 4.60 & -2.80 & --- & --- \\
\hline MON & --- & --- & --- & --- & 6.24 & -2.91 & 6.28 & -3.24 & 5.71 & -3.24 & 6.61 & -2.85 \\
\hline
\end{tabular}


Table 10.--Maximum and minimum daily water levels for Alsea River at Kozy Kove near Waldport, river mile 11.2--Continued

GAGE HEIGHT, FEET, WATER YEAR OCTOBER 1989 TO SEPTEMBER 1990

\begin{tabular}{|c|c|c|c|c|c|c|c|c|c|c|c|c|}
\hline \multirow[b]{2}{*}{ DAY } & \multicolumn{2}{|c|}{ OCTOBER } & \multicolumn{2}{|c|}{ NOVEMBER } & \multicolumn{2}{|c|}{ DECEMBER } & \multicolumn{2}{|c|}{ JANUARY } & \multicolumn{2}{|c|}{ FEBRUARY } & \multicolumn{2}{|c|}{ MARCH } \\
\hline & MAX & MIN & MAX & MIN & MAX & MIN & MAX & MIN & MAX & MIN & MAX & MIN \\
\hline 1 & 5.21 & -2.19 & 4.96 & -2.36 & 5.45 & -1.97 & 5.37 & -1.57 & 7.61 & 2.74 & 6.70 & .04 \\
\hline 2 & 5.09 & -2.45 & 5.39 & -2.16 & 5.95 & -1.96 & 4.94 & -1.56 & 7.63 & 2.25 & --- & --- \\
\hline 3 & 4.55 & -2.56 & 5.31 & -2.16 & 6.07 & -1.59 & 4.65 & -1.55 & 7.43 & 2.19 & 5.64 & -.89 \\
\hline 4 & 4.36 & -2.48 & 4.83 & -1.99 & 5.93 & -1.15 & 5.68 & -1.53 & 6.56 & 1.73 & 5.55 & -.25 \\
\hline 5 & 4.38 & -2.51 & 4.13 & -1.93 & 6.99 & 1.75 & 5.81 & -1.01 & 6.99 & 1.96 & 5.25 & -.55 \\
\hline 6 & 3.71 & -2.40 & 3.72 & -2.08 & 5.26 & .76 & 6.74 & .70 & 7.67 & 1.83 & 4.89 & -.67 \\
\hline 7 & 3.24 & -2.59 & 4.01 & -1.88 & 5.64 & -.18 & 11.23 & 5.79 & 6.91 & 1.71 & 5.73 & -.70 \\
\hline 8 & 3.09 & -2.55 & 4.32 & -2.05 & 6.49 & -.08 & 10.46 & 5.74 & 8.01 & 1.42 & 5.40 & -.77 \\
\hline 9 & 3.27 & -2.58 & 5.03 & -1.83 & 6.53 & -.80 & 10.24 & 4.15 & 7.96 & 5.32 & 5.61 & -.53 \\
\hline 10 & 3.49 & -2.64 & 5.71 & -2.24 & 6.45 & -1.36 & 8.19 & 3.61 & 7.76 & 5.18 & 6.11 & 1.01 \\
\hline 11 & 3.73 & -2.74 & 6.19 & -2.23 & 6.47 & -1.73 & 7.47 & 1.84 & 6.67 & 3.46 & 5.77 & .47 \\
\hline 12 & 4.81 & -2.63 & 7.00 & -2.10 & 6.83 & -1.82 & 7.05 & .70 & 5.99 & 2.00 & 5.61 & -.12 \\
\hline 13 & 5.43 & -2.61 & 6.82 & -2.20 & 6.64 & -1.81 & 6.87 & .02 & 6.00 & 1.14 & 4.95 & -.48 \\
\hline 14 & 5.71 & -2.59 & 7.29 & -2.19 & 6.56 & -1.80 & 5.61 & -.57 & 4.70 & .20 & 5.92 & -.25 \\
\hline 15 & 6.25 & -2.58 & 6.41 & -2.17 & 6.17 & -1.78 & 5.22 & -.60 & 5.07 & .10 & 4.85 & -.86 \\
\hline 16 & 6.34 & -2.57 & 6.16 & -2.17 & 5.68 & -1.78 & 5.62 & -.63 & 5.67 & .75 & 4.44 & -.86 \\
\hline 17 & 6.68 & -2.55 & 5.55 & -2.15 & 5.29 & -1.77 & 4.79 & -.89 & 4.76 & .71 & 4.64 & -.79 \\
\hline 18 & 5.98 & -2.55 & 4.68 & -2.11 & 5.17 & -1.59 & 3.74 & -1.23 & --- & --- & 3.91 & -.87 \\
\hline 19 & 5.90 & -2.54 & 4.12 & -2.13 & 4.51 & -1.71 & 4.45 & -.87 & --- & --- & 3.73 & -.87 \\
\hline 20 & 5.38 & -2.24 & 4.42 & -2.08 & 4.65 & -1.39 & 4.32 & -.97 & --- & --- & 3.36 & -.87 \\
\hline 21 & 4.68 & -1.90 & 4.17 & -1.87 & 4.68 & -1.27 & 4.88 & -.97 & --- & --- & 3.00 & -.88 \\
\hline 22 & 5.00 & -1.59 & 4.41 & -1.62 & 4.77 & -1.68 & 5.22 & -.97 & --- & --- & 3.52 & -.88 \\
\hline 23 & 5.39 & -1.28 & 5.52 & -1.37 & 4.93 & -1.68 & 5.22 & -.97 & --- & --- & 3.95 & -.88 \\
\hline 24 & 4.86 & -1.57 & 5.76 & -1.35 & 4.84 & -1.67 & 5.07 & -.97 & --- & --- & 4.41 & -.89 \\
\hline 25 & 4.69 & -1.66 & 6.23 & -1.30 & 5.13 & -1.65 & 6.01 & -.97 & --- & --- & 4.94 & -.89 \\
\hline 26 & 5.14 & -1.63 & 5.94 & -1.71 & 6.02 & -1.64 & 6.52 & -.31 & $\ldots$ & --- & 5.14 & -.89 \\
\hline 27 & 4.75 & -2.16 & 5.23 & -2.02 & 6.26 & -1.63 & 6.68 & .46 & --- & --- & 5.34 & -.90 \\
\hline 28 & 4.49 & -2.41 & 5.48 & -2.01 & 5.94 & -1.61 & 7.68 & 1.87 & --- & --- & 5.87 & -.90 \\
\hline 29 & 4.68 & -2.40 & 5.49 & -1.99 & 5.28 & -1.60 & 7.96 & 3.23 & --- & --- & 6.00 & -.90 \\
\hline 30 & 4.83 & -2.39 & 5.87 & -1.98 & 5.27 & -1.60 & 8.37 & 3.97 & --- & --- & 5.83 & -.90 \\
\hline 31 & 5.08 & -2.37 & --- & --- & 5.69 & -1.59 & 7.87 & 2.83 & --- & --- & 5.72 & -.91 \\
\hline MON' & 6.68 & -2.74 & 7.29 & -2.36 & 6.99 & -1.97 & 11.23 & -1.57 & --- & --- & --- & --- \\
\hline
\end{tabular}


Table 10.--Maximum and minimum daily water levels for Alsea River at Kozy Kove near Waldport, river mile 11.2--Continued

GAGE HEIGHT, FEET, WATER YEAR OCTOBER 1989 TO SEPTEMBER 1990

\begin{tabular}{|c|c|c|c|c|c|c|c|c|c|c|c|c|}
\hline \multirow[b]{2}{*}{ DAY } & \multicolumn{2}{|c|}{ APRIL } & \multicolumn{2}{|c|}{ MAY } & \multicolumn{2}{|c|}{ JUNE } & \multicolumn{2}{|c|}{ JULY } & \multicolumn{2}{|c|}{ AUGUST } & \multicolumn{2}{|c|}{ SEPTEMBER } \\
\hline & MAX & MIN & MAX & MIN & $\operatorname{MAX}$ & MIN & $\operatorname{MAX}$ & MIN & MAX & MIN & MAX & MIN \\
\hline 1 & 5.39 & -.91 & 3.30 & -1.01 & 4.52 & -1.10 & 4.56 & -1.14 & --- & --- & --- & \\
\hline 2 & 4.56 & -.91 & 3.09 & -1.01 & 4.92 & -1.11 & 4.46 & $-1 \cdot 14$ & --- & -- & -- & \\
\hline 3 & 3.81 & -.92 & 3.22 & -1.01 & 4.96 & -1.11 & 4.83 & -1.14 & --- & --- & --- & \\
\hline 4 & 3.51 & -.92 & 3.95 & -1.02 & 5.08 & -1.11 & 5.43 & -1.14 & --- & $--\infty$ & --- & \\
\hline 5 & 3.98 & -.92 & 4.15 & -1.02 & 5.18 & -1.12 & 5.53 & -1.14 & --- & --- & --- & \\
\hline 6 & 4.22 & -.93 & 4.48 & -1.02 & 4.98 & -1.12 & 5.63 & -1.14 & --- & --- & --- & \\
\hline 7 & 4.40 & -.93 & 4.48 & -1.02 & 4.88 & -1.12 & 5.69 & -1.14 & --- & --- & --- & \\
\hline 8 & 4.67 & -.93 & 4.34 & -1.03 & 4.94 & -1.13 & 5.56 & -1.14 & --- & --- & --- & \\
\hline 9 & 4.70 & -.94 & 4.44 & -1.03 & 5.10 & -1.13 & 5.39 & -1.14 & $--\infty$ & $--\infty$ & $--\infty$ & \\
\hline 10 & 4.76 & -.94 & 4.90 & -1.03 & 4.84 & -1.13 & --- & $--\infty$ & --- & --- & --- & \\
\hline 11 & 5.33 & -.94 & 4.79 & -1.04 & 4.70 & -1.14 & --- & --- & --- & --- & --- & \\
\hline 12 & 4.79 & -.95 & 4.56 & -1.04 & 4.43 & -1.14 & $--\infty$ & $--\infty$ & $-\infty$ & --- & --- & \\
\hline 13 & 4.68 & -.95 & 4.13 & -1.04 & 4.06 & -1.14 & -- & $--1-$ & -- & $-\infty$ & --- & \\
\hline 14 & 4.55 & -.95 & 3.85 & -1.05 & 3.63 & -1.14 & --- & --- & --- & --- & --- & \\
\hline 15 & 4.35 & -.95 & 3.55 & -1.05 & 3.69 & -1.14 & -- & -- & $-\infty$ & --- & -- & \\
\hline 16 & 4.07 & -.96 & 2.92 & -1.05 & 4.43 & -1.14 & $--\infty$ & $-d-$ & $-\infty$ & $--\infty$ & --- & \\
\hline 17 & 3.67 & -.96 & 2.71 & -1.06 & 4.69 & -1.14 & --- & $-a-$ & -- & --- & --- & \\
\hline 18 & 3.04 & -.96 & 3.27 & -1.06 & 4.73 & -1.14 & --- & --- & --- & --- & --- & \\
\hline 19 & 3.60 & -.97 & 5.21 & -1.06 & 5.26 & -1.14 & --- & $\rightarrow$ & $--\infty$ & --- & -- & \\
\hline 20 & 4.20 & -.97 & 5.33 & -1.07 & 5.83 & -1.14 & --- & --- & --- & --- & --- & \\
\hline 21 & 4.72 & -.97 & 6.36 & -1.07 & 6.56 & -1.14 & --- & $-\infty$ & $-\infty$ & --- & -- & $-\infty$ \\
\hline 22 & 5.99 & -.98 & 6.33 & -1.07 & 6.59 & -1.14 & --- & $-\infty$ & $-\infty$ & --- & -- & -- \\
\hline 23 & 5.99 & -.98 & 6.39 & -1.08 & 6.86 & -1.14 & $-\infty$ & -+- & -- & --- & -- & $-\infty$ \\
\hline 24 & 6.12 & -.98 & 6.52 & -1.08 & 6.53 & -1.14 & $-\infty$ & $-\infty-$ & --- & $-\infty$ & -- & --- \\
\hline 25 & 6.41 & -.99 & 6.45 & -1.08 & 5.86 & -1.14 & --- & $-\infty$ & $-\infty$ & $-\infty$ & --- & \\
\hline 26 & 6.84 & -.99 & 6.59 & -1.09 & 5.09 & -1.14 & $-\infty$ & $-4-$ & $-\infty$ & $--\infty$ & $--\infty$ & \\
\hline 27 & 6.74 & -.62 & 6.74 & -1.09 & 4.69 & -1.14 & $-\infty$ & --- & --- & $-\infty$ & --- & --- \\
\hline 28 & 6.84 & 1.17 & 5.91 & -1.09 & 4.73 & -1.14 & $-\infty-$ & -- & --- & $-\infty$ & $-\infty$ & $-\infty$ \\
\hline 29 & 5.50 & -.57 & 5.01 & -1.10 & 4.76 & -1.14 & --- & -- & -- & $-\infty$ & --- & $-\infty$ \\
\hline 30 & 4.13 & -1.00 & 5.37 & $-1 \cdot 10$ & 4.49 & -1.14 & --- & --- & --- & --- & --- & --- \\
\hline 31 & --- & --- & 5.00 & -1.10 & --- & --- & $--\infty$ & -+- & --- & --- & --- & \\
\hline MONI & 16.84 & -1.00 & 6.74 & -1.10 & 6.86 & -1.14 & --- & --- & --- & -- & $--\infty$ & \\
\hline
\end{tabular}




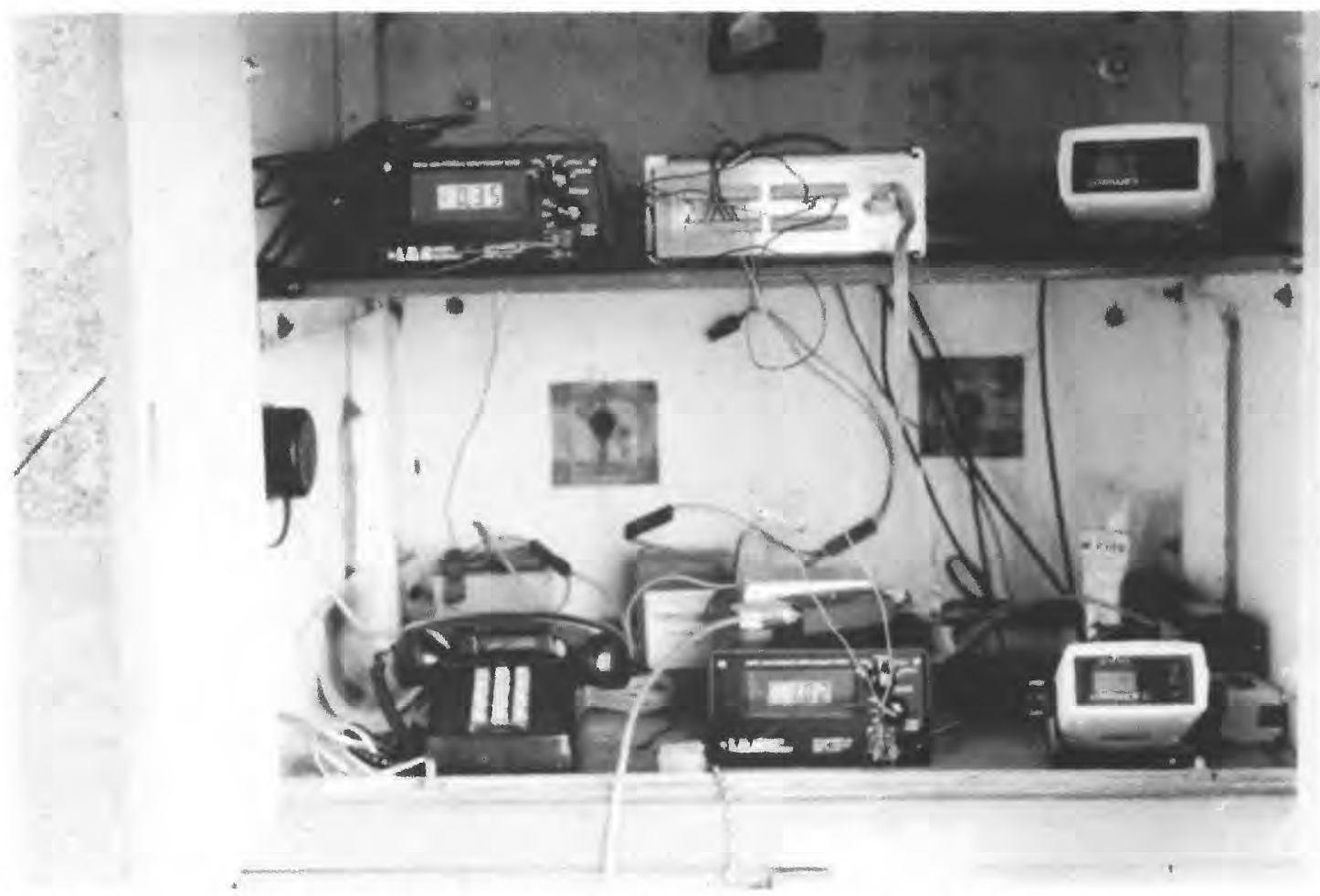

Instrument shelter located on pier 6. Top shelf (left to right) velocity meter, data logger, depth sounder. Bottom shelf, telephone, flood tide velocity meter, and depth sounder.

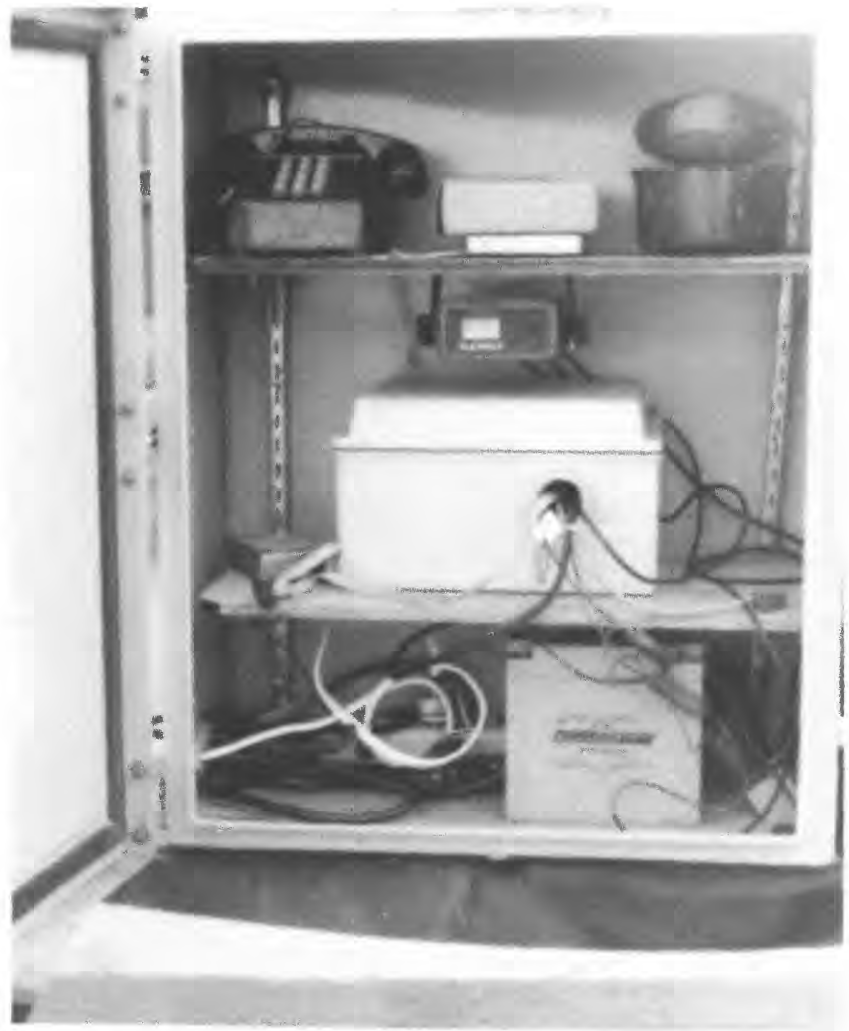

Second instrument shelter located on work trestle. 
Table 11.--Discharge measurements taken in the Alsea River Estuary

\begin{tabular}{|c|c|c|c|c|c|}
\hline LOCATION & DATE & $\begin{array}{l}\text { TIDE } \\
\text { TYPE }\end{array}$ & $\begin{array}{l}\text { DISCHARGE } \\
\text { CUBIC FEET } \\
\text { PER SECOND }\end{array}$ & $\begin{array}{l}\text { AREA } \\
\text { SQUARE } \\
\text { FEET }\end{array}$ & $\begin{array}{l}\text { MEAN VELOCITY } \\
\text { FEET PER } \\
\text { SECOND }\end{array}$ \\
\hline MOUTH RM 0.0 & $4 / 13 / 88$ & EBB & 35,100 & 7,290 & 4.81 \\
\hline MOUTH RM 0.0 & $4 / 14 / 88$ & FLOOD & 38,900 & 8,120 & 4.79 \\
\hline RM 1.1 & $12 / 12 / 89$ & EBB & 42,600 & 19,200 & 2.22 \\
\hline DRIFT CR RM 5.0 & $12 / 13 / 89$ & EBB & 12,000 & 5,300 & 2.26 \\
\hline
\end{tabular}

